\title{
Local Rewetting and Distortion of Paper
}

by

\section{Chris Qun Mao}

\author{
A thesis submitted in conformity with \\ the requirements for the \\ Degree of Master of Applied Science \\ in the Department of Chemical Engineering and Applied Chemistry \\ in the University of Toronto
}

두 Copyright by Chris Qun Mao, 2001 
National Library

of Canada

Acouisitions and Bibliographic Services

395 Wellington Stroet Otawa ON KIA ONA Canada
Bibliothéque nationale

du Canada

Acquisitions et

services bibliographiques

395, rue Wellington

Onawa ON KIA ONA

Canada
The author has granted a nonexclusive licence allowing the National Library of Canada to reproduce, loan, distribute or sell copies of this thesis in microform, paper or electronic formats.
L'auteur a accordé une licence non exclusive permettant à la Bibliothèque nationale du Canada de reproduire, prêter, distribuer ou vendre des copies de cette thèse sous la forme de microfiche/film, de reproduction sur papier ou sur format électronique.

L'auteur conserve la propriété du droit d'auteur qui protège cette thèse. $\mathrm{Ni}$ la thèse ni des extraits substantiels de celle-ci ne doivent être imprimés ou autrement reproduits sans son autorisation.

\section{Canadầ}




\title{
Local Rewetting and Distortion of Paper
}

\author{
by \\ Chris Qun Mao \\ A thesis submitted in conformity with \\ the requirements for the \\ Degree of Master of Applied Science \\ in the Department of Chemical Engineering and Applied Chemistry \\ in the University of Toronto
}

Year of convocation: 2001

\begin{abstract}
Local rewetting may lead to undesirable distortions in the surface of paper. Using an optical surface scanning interferometer and a modified inkjet printer, a technique for the investigation of local distortions was developed. With this technique, tests of various grades of paper were carried out, and the local distortions were quantified as the changes in local surface roughness, skewness, kurtosis, and average surface height. The relationships between local distortions and paper properties are discussed. A model was developed for the prediction of local paper distortion in terms of the change in surface height as a result of local rewetting:

$$
\Delta H=k \frac{R_{a} W}{\sqrt{\left(\frac{T_{b}}{g}\right) \rho}}
$$
\end{abstract}

This model takes into account both the amount of water $(W)$ applied to paper 
and the relevant paper properties including roughness $(R a)$, fiber-to-fiber bond strength $(T b)$, and sheet density $(\rho)$. Correlations show that the model values agree well with the experimental results. 


\section{Acknowledgements}

First and foremost I would like to thank my supervisors, Prof. Jan K. Spelt, Prof. Mark T. Kortschot, and Prof. Ramin Farnood. I appreciate their guidance, patience, and encouragement. My work would not have been possible to complete without their confidence in me and assistance in handing creative solutions to problems. I really feel privileged to work under their supervision.

Dr. Paul Shallhorn at Domtar and Mr. Ivan Gagnon provided me very valuable suggestions, and I would also express my appreciation to them.

My gratitude also goes to Prof. Doug Reeve. I admire not only his academic excellence but his enthusiasm and investment in the education of the professional skills of graduate students. I have benefited a great deal from the various activities organised by Pulp and Paper Centre.

I would also like to acknowledge the Surface Science Consortium. The research would not have been possible without their financial support.

My colleagues have been all very helpful, and I sincerely thank to all of them, especially Dr. Sanjiv Sinha, for his technical support and valuable advice and Cindy Tam for her friendly administrative support and making me feel at home at the Centre.

Especially thanks to my wife Biyu. I appreciate her patience, understanding, kindness, and support. I cannot find any words to express my acknowledgement to my parents, but a special feeling from my mind. My parents, to whom, I am deeply indebted. 


\section{TABLE OF CONTENTS}

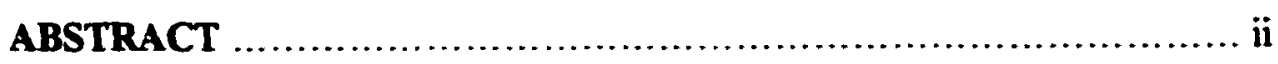

ACKNOWLEDGEMENTS ...............................................

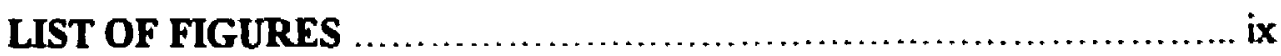

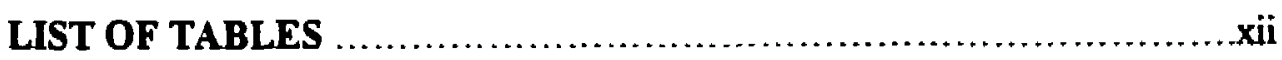

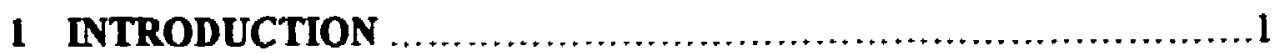

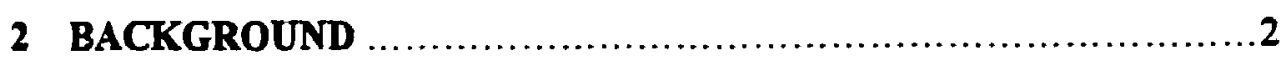

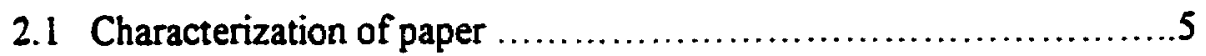

2.1.1 Introduction ..............................................

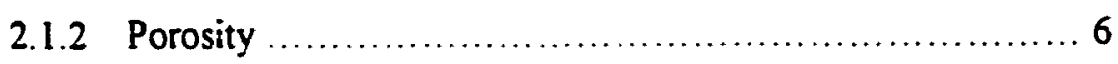

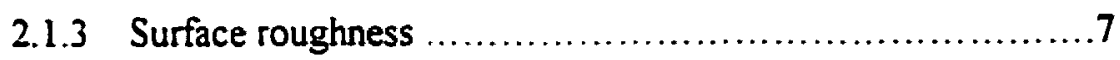

2.1.4 Fiber-to-fiber bonding and bond strength .................12

2.1.5 Formation .......................................... 13

2.2 Effects of water on paper .................................... 15

2.2.1 Water-paper interaction ............................. is

2.2.1.1 Fiber swelling ................................. 15

2.2.1.2 Water absorption and wetting .................. 17

2.2.2 Water-induced distortions ............................ 21

2.2.2.1 Introduction .............................. 21

2.2.2.2 Hygroexpansion ............................. 22

2.2.2.3 Curl ............................................ 24

2.2.2.4 Cockle ....................................... 26

2.2.2.5 Fiber rising and sheet roughening .............. 27

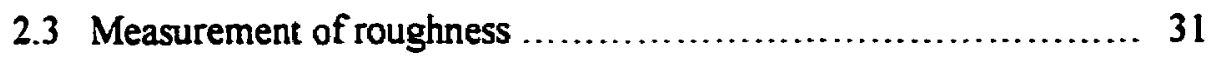

2.3.1 Optical surface profilometry ....................... 31 
2.3.1.1 Confocal laser scanning microscopy ........... 31

2.3.1.2 WYKO optical scanning profilometer ................33

2.3.2 Traditional methods .......................................... 36

2.3.1.1 Air flow methods ............................. 36

2.3.1.2 Mechanical stylus profilometry ................ 38

3 Evaluation of WYKO NT-2000 system $\ldots \ldots \ldots \ldots \ldots \ldots \ldots \ldots \ldots \ldots, 41$

3.1 Introduction ............................................... 41

3.2 WYKO surface scanning profilometry .....................42 42

3.2.1 Experimental setup ................................... 42

3.2.2 Performance of WYKO NT-2000 system .................. 45

3.2.3 Measurement of roughness ........................... 47

3.3 Mechanical stylus profilometry ............................... 48

3.3.1 Surtronic $3+$ stylus profilometer ....................... 48

3.3 .2 Measurement of roughness ............................ $\$ 1$

3.4 Experimental results and comparison ......................... 51

3.5 Summary ................................................ 59

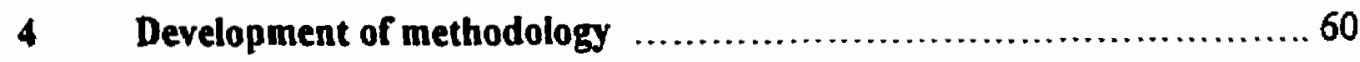

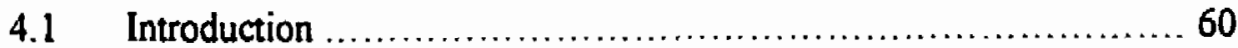

4.2 Local rewetting methodology ............................. 61

4.2.1 Previous work ........................................ 61

4.2.2 Modification of an inkjet printer ..................... 62

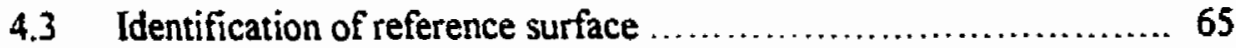

4.3.1 Preparation of specimens ............................. 65

4.3.2 Testing procedure ....................................... 68

4.3.3 Experimental results .................................. 74

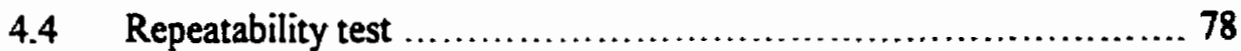

4.5 Investigation of local distortion .................................. 79

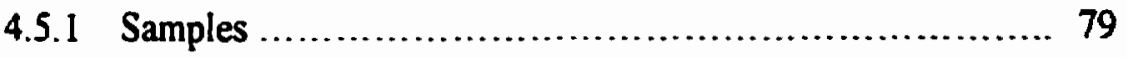




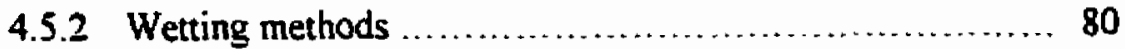

4.5.3 Experimental procedure $\ldots \ldots \ldots \ldots \ldots \ldots \ldots \ldots \ldots \ldots . . \ldots 2$

5 Measurement of bond strength ................................... 86

5.1 Introduction ................................................... 86

5.2 Preparation of specimens ........................................ 86

$5.3 \quad$ Experimental setup ........................................ 88

5.4 Measurement of bond-breaking energy .......................... 89

$5.5 \quad$ Bond strength .................................................. 90

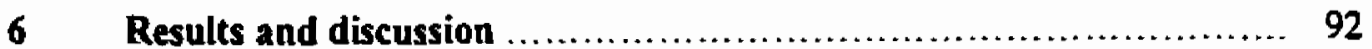

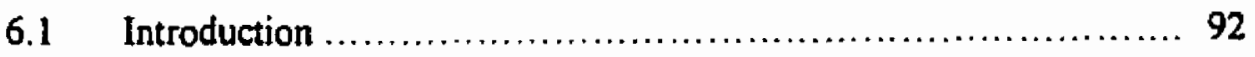

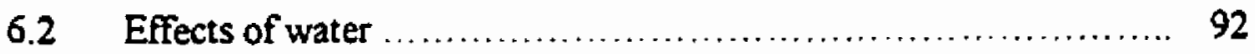

6.2 .1 Changes in roughness ............................... 92

6.2.2 Changes in skewness, and kurtosis .................... 96

6.2 .3 Changes in average step height $\ldots \ldots \ldots \ldots \ldots \ldots \ldots \ldots \ldots . \ldots \ldots$

6.2.4 Effect of amount of water ............................ 103

$6.3 \quad$ Effects of paper properties ................................. 107

6.3 .1 Roughness .......................................... 107

6.3 .2 Bond strength .................................... 109

6.3 .3 Density ........................................ 111

7 Modelling the change in average step height $\ldots \ldots \ldots \ldots \ldots \ldots \ldots \ldots \ldots, 114$

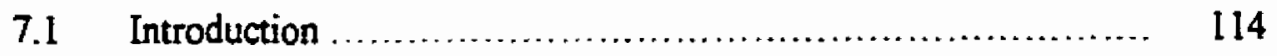

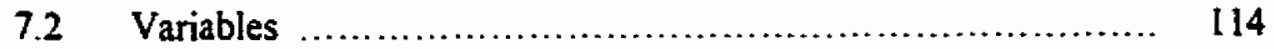

7.3 Establishment of the model ............................... 116

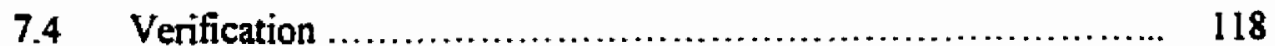

8 Conclusions and recommendations .............................. 121

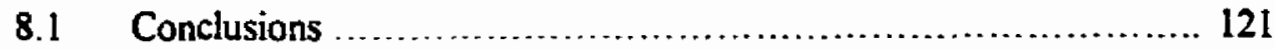


8.2 Recommendations .......................................... 123

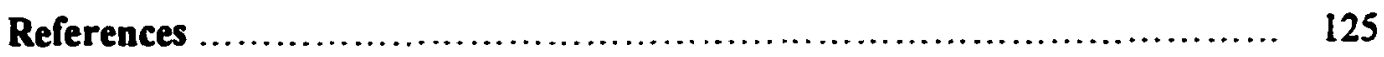

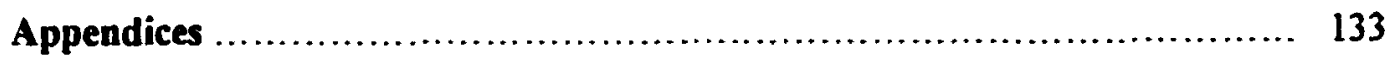

l. Roughness $(\mathrm{Ra}, \mu \mathrm{m})$ measured using WYKO ${ }^{\text {TM }}$ NT-2000 system ........... 133

2. Roughness $(\mathrm{Ra}, \mu \mathrm{m})$ measured using stylus scanning profilometry ........ $\quad 134$

3. Experimental data of WYKO repeatability test ......................... 135

4. Fiber-to-fiber strength $\left(\mathrm{J} / \mathrm{m}^{2}\right)$ of uncoated papers ...................... 135

5. Changes in roughness $(\mathrm{Ra}, \mathrm{Rq}, \mu \mathrm{m})$ and average step height due to pure water rewetting ........................................... 136

6. Changes in roughness and average step height due to rewetting of

2 -time printing ...................................................... 136

7. Changes in roughness, skewness, kurtosis, and average step height due to 2-point line rewetting ......................................... 138

8. Changes in roughness, skewness, kurtosis, and average step height due to $50 \mu \mathrm{m}$ droplet rewetting 142 


\section{LIST OF FIGURES}

Fig. 2.1. Successive decrease in the bonding between cellulosic fibrils caused by water absorption from $A$ to $D$; or increase in the

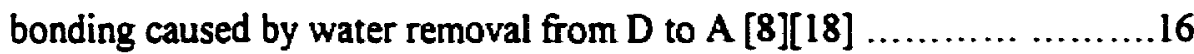

Fig. 2.2. Ways in which water may be held by fibers [35] ...................... 18

Fig. 2.3. Hygroexpansional behavior of paper under humidity conditions [40] ….23

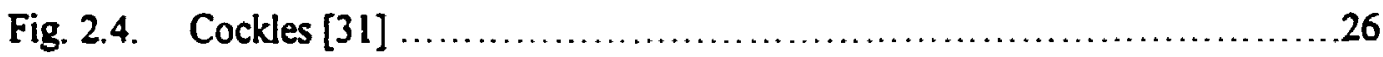

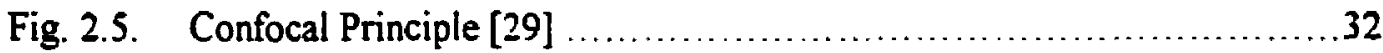

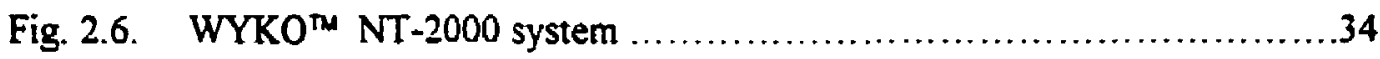

Fig. 2.7. Section of part of a Print-Surf head, showing themeasuring

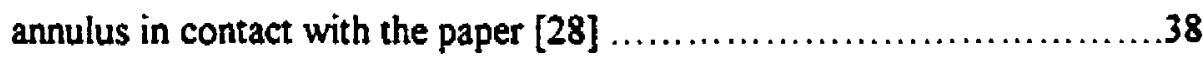

Fig. 3.1 Experimental setup (WYKO ${ }^{\text {TM }}$ NT-2000 system) ........................43

Fig. 3.2. The Surtronic $3+$ stylus profilometer ...................................49 49

Fig. 3.3. The pick-up of the Surtronic $3+$ stylus profilometer ....................... 50

Fig. 3.4. The relationship between roughness of coated paper

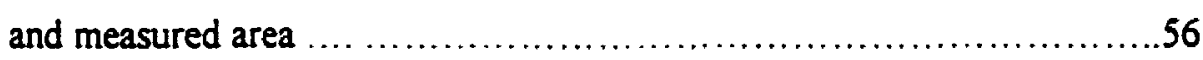

Fig. 3.5. The relationship between roughness of uncoated paper and measured area .56

Fig. 3.6. The relationship between standard deviation of roughness of coated paper and measured area .57

Fig. 3.7. The relationship between standard deviation of roughness of uncoated paper and measured area .................................57

Fig. 3.8. Typical WYKO image, MWC-WO …..................................58

Fig. 3.9. Typical WYKO image, TMP No.2 ..................................58

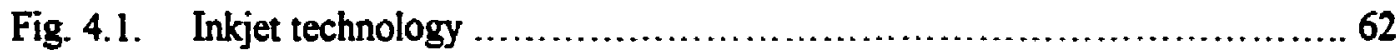

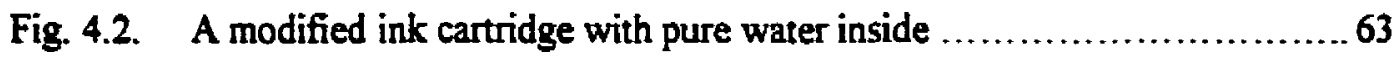

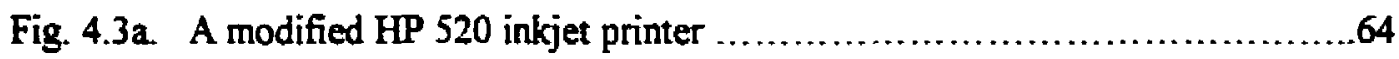

Fig. 4.3b. Paper specimen with glass microscope slide inserted in the sample slot ....65 


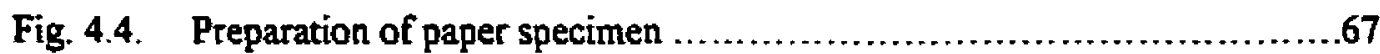

Fig. 4.5. Metal shim as reference taped at its two ends onto paper surface .........67 67

Fig. 4.6. The locations of 5 reference areas and the captured paper surface .........71

Fig. 4.7. Reference testing, left is reference, and right is paper surface ............ 72

Fig. 4.8. Captured areas of reference surface and paper surface ...................72

Fig. 4.9. Average step height between the surface of reference and the surface of paper specimen .............................................73

Fig. 4.10. Transparent tape raised by internal forces caused by fiber swelling as a

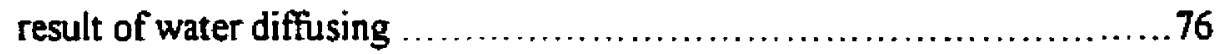

Fig. 4.11. WYKO image. The left is reference; the right is paper surface ............ 84

Fig. 4.12. WYKO image of two captured area .................................. 84

Fig. 5.1. Preparation of specimen for the measurement of bond strength ............87

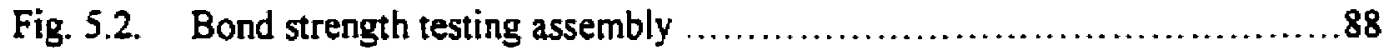

Fig. 5.3. Graph of the force required for specimen delamination ...................89

Fig. 6.1. Changes in roughness vs. types of paper (2-point line rewetting, wetting liquid: ink-dyed water ( $20 \%$ ink); captured area: $320 \times 320 \mu \mathrm{m})$

Fig. 6.2. Changes in roughness vs. types of paper ( $50 \mu \mathrm{m}$ droplet rewetting, wetting liquid: ink-dyed water ( $20 \%$ ink); captured area: $80 \times 80 \mu \mathrm{m})$.

Fig. 6.3. Skewness before rewetting vs. types of paper (For 2-point line rewetting, the captured area was $320 \times 320 \mu \mathrm{m}$; for $50 \mu \mathrm{m} \mathrm{D}$ droplet rewetting, the captured area was $80 \times 80 \mu \mathrm{m}$ )

Fig. 6.4. Change in skewness due to rewetting vs. types of paper. (For 2-point line rewetting, the captured area was $320 \times 320 \mu \mathrm{m}$; for $50 \mu \mathrm{m}$ droplet rewetting, the captured area was $80 \times 80 \mu \mathrm{m}$ ) 97

Fig. 6.5. Kurtosiss before rewetting vs. types of paper (For 2-point line rewetting, the captured area was $320 \times 320 \mu \mathrm{m}$; for $50 \mu \mathrm{m}$ droplet rewetting, the captured area was $80 \times 80 \mu \mathrm{m}$ ) 
Fig. 6.6. Change in kurtosis due to rewetting vs. types of paper (For 2-point line rewetting, the captured area was $320 \times 320 \mu \mathrm{m}$; for $50 \mu \mathrm{m}$ droplet rewetting, the captured area was $80 \times 80 \mu \mathrm{m}$ ) 99

Fig. 6.7. Changes in average step height vs. types of paper (For 2-point line rewetting, the captured paper surface was $320 \times 320 \mu \mathrm{m}$, and the captured reference was $320 \times 160 \mu \mathrm{m}$. For $50 \mu \mathrm{m}$ droplet rewetting, the captured area of both captured paper surface and reference was $80 \times 80 \mu \mathrm{m})$ 100

Fig. 6.8. Changes in average step height vs. water diffusion coefficient; (50 $\mu \mathrm{m}$ droplet rewetting) 107

Fig. 6.9. Cahnges in average step height vs. $\mathrm{Ra}$ (2-point line printing rewetting; uncoated paper: IS, newsprint, TMP No.l) 108

Fig. 6.10. Changes in average step height vs. $R a(50 \mu \mathrm{m}$ droplet rewetting; uncoated paper: IS, newsprint, TMP No.I) $\cdot 108$

Fig. 6.11. Changes in average step height vs. bond strength (2-point line printing rewetting)

Fig. 6.12. Changes in average step height vs. bond strength ( $50 \mu \mathrm{m}$ droplet rewetting) .110

Fig. 6.13. Changes in average step height vs. paper density (2-point line printing rewetting)

Fig. 6.14. Changes in average step height vs. paper density ( $50 \mu \mathrm{m}$ droplet rewetting)

Fig. 7.1. Correlation between model values and experimental results (2-point line rewetting)

Fig. 7.2. Correlation between model values and experimental results ( $50 \mu \mathrm{m} \mathrm{D}$ droplet rewetting) 


\section{LIST OF TABLES}

Table 2.1. Air flow roughness measurement methods and their characteristics .....36

Table 2.2. Average arithmetic roughness of coated and uncoated papers ...........39

Table 3.1. Magnifications, field of view, and in-plane resolutions of the WYKO ${ }^{\text {TM }}$ NT-2000 system. ....................................46

Table 3.2. Paper samples and their suppliers ................................48

Table 3.3. Roughness -- comparison among WYKO, mechanical profilometry, and PPS .52

Table 4.1. The changes in local surface height using different areas (transparent tape) as references .75

Table 4.2. The changes in local surface height using different areas (metal shim) as references

Table 4.3. WYKO repeatability test (Sample: TMP No.1. Experiments: 10. Scanning area: $603 \times 459 \mu \mathrm{m}$; captured paper area: $320 \times 320 \mu \mathrm{m}$ ) $\ldots .79$

Table 4.4. Samples and some relevant properties ........................ 80

Table 5.1. Fiber-to-fiber bond strength of uncoated paper ....................... 90

Table 6.1. T-test results of changes in roughness due to rewetting on different grades of paper 95

Table 6.2. Effect of using different wetting liquids on the changes of average step height as well as roughness 102

Table 6.3. Results of local distortions caused by the different amount of water applied to paper

Table 6.4. Size of dots of ink-dyed water (font 1 ) on different grades of paper and the amount of water absorbed by paper as well as the water diffusion coefficient. 


\section{CHAPTER ONE}

\section{INTRODUCTION}

The interactions between paper and water in rewetting processes such as coating and printing may lead to undesirable distortions in the paper structure since paper is susceptible to water. For instance, coating provides an excellent means of improving the surface of paper in terms of both optical and printability properties. However, when an aqueous suspension such as coating color is applied to base paper, fiber swelling and debonding may occur, particularly with papers containing mechanical pulps, influencing the coating coverage and consequent printability [1]. In offset lithographic printing, fountain water transfers to the non-image areas of the printing plate to keep these areas free from ink. Water then transfers from the plate via the blanket to the paper. Therefore, the water is absorbed into the paper and causes dimensional changes such as fiber rising and sheet roughening [2]. In flexographic printing and inkjet printing, water-based inks are applied to paper for the image areas, which may also result in distortions on paper.

Many studies have focussed on the phenomena of large-scale distortions in paper such as hygroexpansion, curl, waviness, and cockle [3-6]. However, there are few studies of small-scale distortions caused by localized rewetting. The obstacles are the diversity of these phenomena, the complexity of paper structure, and the 
limitation of experimental methodology.

It has been recognized that dimensional instability represents the susceptibility of paper to changes of dimensions with changes in water content. Basically, the root cause of these changes in dimensions lies in the change in dimensions of the fibers of which the paper is composed [3]. Furthermore, the following were considered to be three potential fundamental causes of fiber rising due to rewetting: swelling of fibers, dissolution or weakening of fiber-to-fiber bonds, and release of internal stresses in the paper structure [16]. Nevertheless, many aspects of this problem are still imperfectly understood. There is a need to better understand the rewetting process and the distortions it creates in paper. Such knowledge would be helpful to identify avenues for the improvement of paper quality and printability.

It was hypothesised that, during local rewetting, the more water applied to paper, the greater the change in local paper surface structure and that paper properties such as the initial roughness of the local surface to be wetted, fiber-to-fiber bond strength, and sheet density play important roles in local distortions when paper is locally wetted.

The objectives for this study were: first, to investigate the local distortions caused by local rewetting; and second, to develop a model for the prediction of local distortion on different grades of paper as a result of local rewetting. 
To realize above objectives, a non-contact optical scanning profilometer, WYKO ${ }^{\text {MM }}$ NT-2000 system, was evaluated and applied in this study. WYKO can scan a sample at ambient atmosphere, which makes it possible to investigate the paper specimens under dry and wet conditions. Compared with confocal laser scanning microscopy [29], WYKO has a wide range of scanning depth and high resolutions both in the in-plane and out-of-plane directions. The principle behind the WYKO is light interference. WYKO's Vision-32 ${ }^{\text {TM }}$ software provides a variety of analysis capabilities including 2D and 3D maps and many surface parameters such as arithmetic average roughness $\left(R_{a}\right)$, root mean square roughness $\left(R_{q}\right)$, skewness $\left(R_{s k}\right)$, kurtosis $\left(\mathbf{R}_{\mathbf{k u}}\right)$, and average step height.

In this thesis, Chapter Two introduces the background, which contains the characterization of paper, literature review, and the methodology related to this research. The evaluation of the WYKO ${ }^{\text {TM }}$ NT 2000 system is described in Chapter Three; WYKO was compared to two conventional methods: Parker Print Surf (PPS) method and mechanical stylus profilometry. Chapter Four introduces the development of experimental methodology as well as the modification of an inkjet printer, which was used for the local rewetting of samples. Subsequently, the experimental procedure for the investigation of local distortions caused by local rewetting is detailed. Since fiber-to-fiber bonding was considered to have fundamental influence on paper distortions such as fiber rising and sheet roughening bond strength was measured with relevant samples, as described in Chapter Five. The WYKO 
experimental results are presented in Chapter Six. Quantitative analysis, statistical tests are conducted, and the relationships between local distortions and paper properties are discussed. Based on the experimental results, an empirical model has been developed, which is described in Chapter Seven. The model takes into account both the amount of water applied to paper and paper properties in terms of arithmetic average roughness, bond strength, and sheet density. The conclusions and suggestions for the future work are made in Chapter Eight. 


\section{CHAPTER TWO}

\section{BACKGROUND}

\section{I CHARACTERIZATION OF PAPER}

\subsubsection{INTRODUCTION}

Paper is made of cellulosic fibers and therefore, the characteristics of individual fibers play an important role in papermaking and paper end-use. It was concluded that the root cause of fiber rising, sheet roughening, and related distortions caused by water lies in the swelling or change in dimensions of the fibers of which the paper is composed [3]. However, the phenomena of distortions related to water are dependent not only on the mechanisms of individual fiber swelling but also on the structure of the network and its properties. The microstructure of a self-bonding network of cellulosic fibers [7] and its properties such as roughness, fiber-to-fiber bonding, fiber orientation, formation (a measure of uniformity; see Section 2.1.5), and density have significant effects on paper distortions when paper contacts water. For instance, if the structure and composition of paper varies from one side of paper to the another, then the degree and direction of the dimensional instability will vary accordingly, resulting in a departure from the flat form; i.e, the well known phenomenon of curl. It is difficult to involve all the factors for the interpretation of 
paper distortions because paper is a complex material. In Section 2.1.2 to 2.1.5, paper is characterized in terms of porosity, surface roughness, fiber-to-fiber bond strength, and formation, all of which influence the rewetting and consequent distortions of paper. The characteristics of fiber swelling and relevant examples of distortions including fiber rising, sheet roughening, hygroexpansion, curl, and cockle are discussed in Section 2.2 .

\subsubsection{POROSITY}

Paper is a highly porous material compared to other materials such as plastics and metal. It is noted that ordinary papers are about $50 \%$ air by volume. Some of the air is present inside the fibers, but most of it resides in pores within the sheet structure. The ratio of pore volume to total volume of sheet is called the porosity of the sheet [31].

The property of paper porosity, although being fundamentally important, is rarely measured in paper except occasionally in laboratory studies. However, a related property, air permeability, is often used. Air permeability is defined as the ability of a paper to allow air to flow through it under a pressure difference across the sheet [31]. It is structure-related property depending on the number, size, shape, and distribution of the pores in a sheet. 
Porosity plays a fundamental role in water absorption in paper. For example, it was reported that a large decrease in water permeability was observed for decreasing porosity. The decrease was more pronounced for chemical pulp than mechanical pulp [52]. As well, an experiment indicated that porosity and sizing level strongly influenced the initial liquid-sheet contact angle and duration of wetting delay [32]. It was concluded that porous papers have greater dimensional stability with moisture change since there is more space or internal volume available for expansion and contraction of the fibers. Papers of lesser porosity, that is denser papers, do not have as much internal air volume for inward compensations of fiber changes [12].

Generally speaking, a paper made exclusively from long fibers will be more porous than a paper made from part long and part short or filler types of fibers under the same conditions. The short fibers fill in some of the voids created by the long fibers and therefore reduce the air volume. Calendering at the end of paper machine lowers paper porosity. Surface sizing and coating also decrease the porosity, particularly at the surface.

\subsubsection{SURFACE ROUGHNESS}

Surface roughness of paper or board affects the amount of coating necessary and the absorption of water or ink. For paper coating, a rough base paper requires 
more coating to cover the surface of the stock. Roughness is especially significant in printing paper. For example, in offset printing, water applied to the non-image area may result in fiber rising on rough paper [16]. As well, oil-based ink or water-based ink may not be uniformly distributed on the image area because of a rough surface.

Roughness has three components in terms of the in-plane resolution [8]: macro roughness at $0.1 \mathrm{~mm}-1 \mathrm{~mm}$; micro roughness at $1 \mu \mathrm{m}-100 \mu \mathrm{m}$; and optical roughness at length $<\lfloor\mu \mathrm{m}$. Macro roughness is the result of formation. Micro roughness primarily relates to the shapes and positions of fibers and fines in the network structure. Macro and micro roughness influence paper gloss and paper uniformity. Optical roughness depends on the surface properties of individual pigment particles and pulp fibers.

Arithmetic average roughness $\left(R_{a}\right)$ and root mean square roughness $\left(R_{q}\right)$ are the most two common measures of surface roughness. The $R_{a}$ for $2 D$ is:

$$
R_{a}=\frac{1}{L} \int_{x=0}^{L}\left|Z_{x}-Z_{0}\right| d x
$$

The $R_{q}$ for $2 D$ is: 


$$
R_{q}=\sqrt{\frac{1}{L} \int_{x=0}^{L}\left(Z_{x}-Z_{0}\right)^{2} d x}
$$

where, $L$ is the length of the measured mean line,

$Z_{x}$ is the local surface height,

$Z_{0}$ is the mean height.

For the 3D $R_{a}$ :

$$
R_{a}=\frac{1}{L W} \int_{y=0}^{W} \int_{x=0}^{L}\left|Z_{x y}-Z_{0}\right| d x d y
$$

For the $3 D R_{q}$ :

$$
R_{q}=\sqrt{\frac{1}{L W} \int_{y=0}^{W} \int_{x=0}^{L}\left(Z_{x y}-Z_{0}\right)^{2} d x d y}
$$

where, $L$ is the length of the measure window,

$W$ is the width of the measure window,

$Z_{x y}$ is the local surface height, 
$Z_{0}$ is the mean surface height.

In practice, the roughness of a paper surface is usually measured indirectly by means of an air flow method such as Parker Print Surf (PPS) or Bendtsen, which is discussed in Section 2.3.2. Because PPS roughness can be converted into mean gap between the PPS head and paper surface, sometimes it is equivalent to the profilometric roughness [27,28]. In this study, an optical profilometer, WYKO ${ }^{\text {IM }}$ NT2000 system, was employed for the measure of roughness of different grades of paper, and the experimental results were compared with the roughness measured with the PPS air flow method and mechanical stylus profilometry as well.

In addition to arithmetic average roughness and root mean square roughness, skewness and kurtosis are sometimes used to characterize the paper surface. The skewness $\left(\mathbf{R}_{\text {sk }}\right)$ and kurtosis $\left(\mathbf{R}_{\mathrm{ku}}\right)$ associated with a profile, are given by the following equations:

$$
\begin{aligned}
& R_{s k}=\frac{1}{R_{q}{ }^{3}} \frac{1}{N} \sum_{i=1}^{N}\left(Z_{i}-Z_{0}\right)^{3} \\
& R_{k u}=\frac{1}{R_{q}{ }^{4}} \frac{1}{N} \sum_{i=1}^{N}\left(Z_{i}-Z_{0}\right)^{4}
\end{aligned}
$$


where, $R_{q}$ is root mean square roughness

$Z_{i}$ is the local surface height,

$Z_{0}$ is the height of the mean line or mean surface.

Skewness represents a measure of asymmetry of the distribution density of profile deviation. The sign of the skewness will tell whether the farther points are proportionately above (positive skewness) or below (negative skewness) the mean surface level. Thus, the predominance of bumps or peaks on a surface will have a positive skewness, and the predominance of holes or valleys in a surface will have a negative skewness.

Kurtosis is a measure of the peakedness of the profile about the mean ine. It provides information about the "spikiness" of a surface. The kurtosis is high when a high proportion of the profile heights fall within a narrow range of heights. Thus, kurtosis is also a measure of the randomness of profile heights. Kurtosis values can range from 0 to 8, with a perfectly Gaussian or random surface having a kurtosis of 3. The farther the value is from 3 , the less random (the more repetitive) the surface is. Profiles with fewer high and low extreme points have a kurtosis value less than 3; those with an appreciable number of high and low extremes have a kurtosis value greater than 3. 


\subsubsection{FIBER-TO-FIBER BONDING AND BOND STRENGTH}

The bonding between fibers is conventionally considered to be primarily due to hydrogen bonds, which is a special type of chemical bonding. Van der Waals forces may also contribute. In principle, the hydrogen bonds between fibers are not different from the hydrogen bonds between fibrils in the fiber wall and the hydrogen bonds between glucose units in the cellulosic molecule. While the fibril bonds give fibers their structural rigidity, fiber-to-fiber bonds form a network of fibers and hence paper [8].

In papermaking, fiber-to-fiber bonds form gradually as solids content increases when water is removed from the wet paper web. On the other hand, water applied to paper may lead to fiber-to-fiber debonding, which may result in dimensional distortions in paper. For instance, a study [33] showed that fiber-to-fiber bonding affects hygroexpansivity of paper (see Section 2.2.2.2); increasing the bonded area increases the hygroexpansivity of paper. Fiber-to-fiber bonding is one of the critical factors which determine the weak regions in the sheet [13]. The mechanical properties of paper depend on the bonding degree of the fiber network. If there were too few bonds between fibers, the network would have no cohesion.

The relative bonded area, RBA, is usually used to characterize the degree of bonding in paper. By definition, RBA is the bonded area of fibers divided by their 
total surface area. One method to determine RBA is measuring the light scattering coefficient of paper. This method is based on the assumption that portions of a fiber not bonded to another fiber scatter light, while the bonded portions, which have no interface with air, do not. The relationship between relative bonded area and the scattering coefficients can be described as $[14,15]$ :

$$
R B A=\left(S_{0}-S\right) i S_{0}
$$

Where, $S$ is the scattering coefficient of the sheet,

$S_{0}$ is the scattering conefficient of the fibers of the sheet in a completely unbonded state.

In this study, bond strength, which is defined as the average bond-breaking energy per unit area of sample $[53,54]$, was used to characterize the degree of fiberto-fiber bonding. The technique used to measure bond strength was developed by Skowronski and Bichard [53]. It is based on the controlled delamination of the sample using a specially designed free-rotating wheel to maintain the same geometry throughout the whole measurement under slow, quasistatic conditions.

\subsubsection{FORMATION}


Paper consists of fibers, fiber fragments, fillers, and chemical additives. The uniformity of paper depends highly on the spatial arrangement of its fibers, fines and other components. On a microstructural level, paper is porous and inhomogeneous. The uneven structure of paper can be observed with naked eye under light. The visually apparent nonuniformity relates to the basis weight variation.

Formation is a measure of nonuniformity in a sheet. A more precise definition of formation is the variability of basis weight of paper or grammage distribution $[7,8]$. The coefficient of variation of grammage (CV), which is calculated over square zones of a finite size, is widely used to characterize the formation. Usually, the $\mathrm{CV}$ of grammage of a sheet decreases with an increase in the size of the inspection zones, and the rate of decrease is governed by the fiber geometry as well as the formation [9]. For an ideal random network of fibers, the coefficient of variation of grammage is dependent on fiber length, fiber coarseness, and average grammage. Therefore, the shorter and less coarse fibers, such as hardwoods, generally have better forming characteristics than softwood fibers and thus produce papers with superior formation characteristics $[10,11]$.

For practical purposes, a useful definition of formation is the small-scale grammage variation in the plane of the paper sheet. The definition provides a simple measurement and unambiguous connection to the structure of paper. Other terms of formation are mass distribution, or the distribution of mass density [8]. 
It was reported that sheet formation influences the print quality. The results showed that the coefficient of variation of grammage clearly affected print quality. Poor formation affects the absorption of water or ink through a complex network of interactions and produces nonuniform printing [30]. Since formation is a property which characterizes the nonuniformity of sheet it should have significant influence on local distortions on paper caused by local rewetting.

\subsection{EFFECT OF WATER ON PAPER}

\subsubsection{WATER-FIBER INTERACTION}

\subsubsection{FIBER SWELLING}

Swelling may be defined as the increase in the dimension of a material accompanying absorption of a solution or its vapor. Cellulosic fibers swell under the influence of water. Therefore, distortions or deformations occur in paper when water is applied to it. Experiments have shown that fiber swelling results in irreversible changes in paper thickness and surface roughness [17]. It is well accepted that fiber swelling is the root cause of fiber rising or sheet roughening $[3,6,16]$.

Attempts have been made to explain the mechanism of fiber swelling. 
Scallan [18] proposed a model for the structure of the fiber cell wall. It was composed of sheets of elementary fibrils separated by lamellar spaces of the same order of thickness as the elementary fibril observed under the microscope. According to this model, fibers swell upon water absorption because water molecules penetrate between hydrogen-bonded fibrils in the fiber wall as Figure 2.1 shows. The amount of bonded water increases, and the degree of internal bonding of the fiber wall decreases.

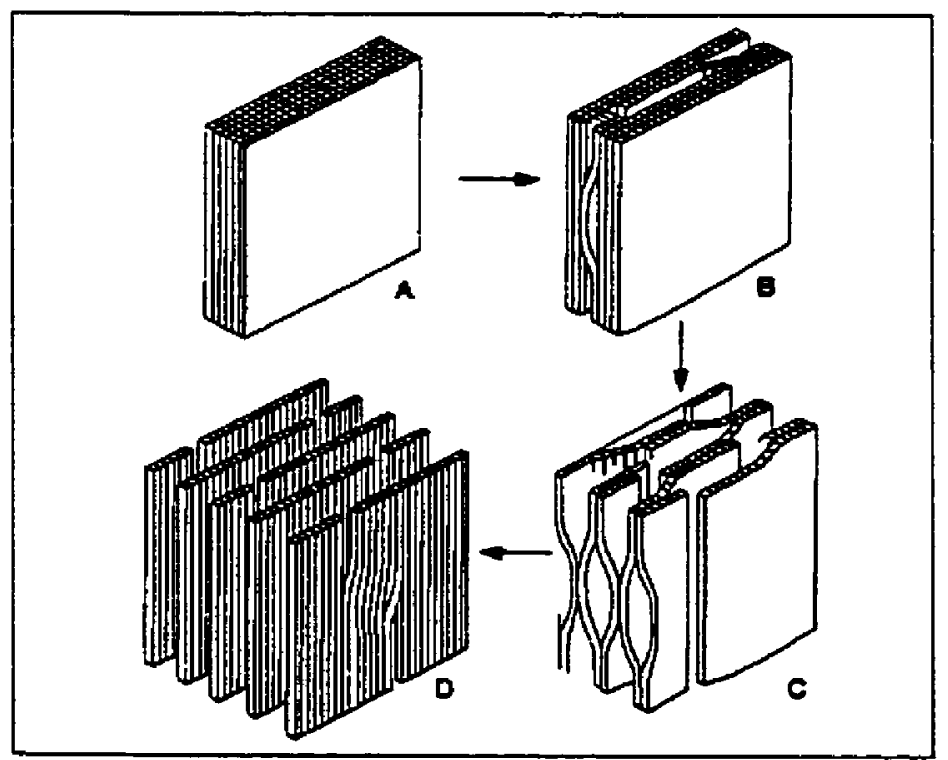

Figure 2.1. Successive decrease in the bonding between cellulosic fibrils caused by water absorption from $A$ to $D$; or increase in the bonding caused by water removal from $D$ to $A[8,18]$

Weise and Paulapuro [34] investigated fiber swelling in situ by means of 
confocal microscopy. In their study, swelling was assumed to be caused by two phenomena: the displacement of morphological features in the fiber cell and submorphlogical swelling caused by bonded water. According to the results for wetimaged fiber cross-sections, the more often fibers were dried and rewetted, the greater the reduction in swelling. It was concluded that fiber drying causes a loss in reswelling due to submorphorlogical changes in the fiber wall.

Beating or refining causes changes in fiber in terms of shortening, external fibrillation, and internal fibrillation [19]. The beating of chemical pulps increases swelling by increasing the delamination of the fiber wall. As a result of beating, the fibers contain much greater quantities of water. The increased amount of water in the cell wall plasticizes the fiber with the result that fiber becomes more flexible and able to conform when laid into a fibrous web by forming processes. However, beating may lead to more distortions in fibers and the web made from these fibers during rewetting since it brings about more swelling in fibers.

\subsubsection{WATER ABSORPTION AND WETTING}

Cellulose and hemicelluloses are substances whose structures are largely due to intra- and inter-molecular hydrogen bonding. This results in attraction to water and so wood fibers are hydrophilic and absorb water readily. 
Stone and Scallan [35] described the water absorbed by pulp fibers with a simple diagram as Figure 2.2 shows. Three fibers are shown lying across one another to form a system in which water can be held in various ways. The walls of the fibers consist of lignin and carbohydrate that can take up water and increase the volume of fibers. This volume is denoted $V_{C}$. The fiber walls enclose lumens, which will hold a certain volume of water $V_{L}$ depending on the morphology of the fibers and the state of lumen collapse. The external surfaces of fibers, particularly after beating, contain a "pile" of microfibrils that can entrap a volume of water $V_{F}$, and finally the interstices between a number of fibers form a capillary system that will hold a volume of water $V_{l}$.

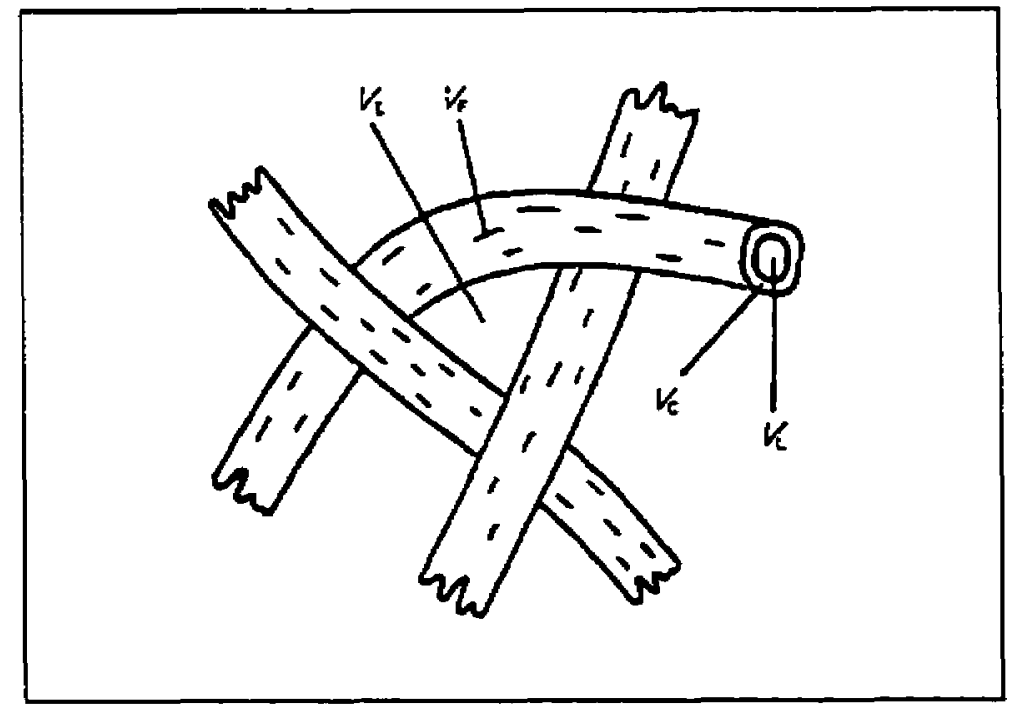

Figure 2.2. Ways in which water may be held by fibers [35] 
Berthod and Desbrieres [20] classified water taken by fibers into two parts. One is free water including inter-fiber free water in the pores between fibers and intra-fiber free water in the lumen of fibers. Another part is bonded water, which is divided into freezing bonded water in the pores of fiber wall and nonfreezing bonded water, chemically bonded to the hydroxylic and carboxylic acid groups in fibers.

Wetting is important in the processes of coating and printing when coating color, fountain solution, and ink need to be transferred to the paper. There are a considerable number of studies that have focused on the mechanism of paper wetting or rewetting [36-39]. However, the wetting process of paper has not been fully understood because of the complexity of paper structure and the many factors that may influence wetting. Nevertheless, some fundamental principle have been revealed.

The interaction of a liquid with a solid surface is governed by three interfacial tension forces: $\gamma_{S v}, \gamma_{\Omega}$, and $\gamma_{L F}$, which are related at equilibrium by Young's equation:

$$
\gamma_{t r} \cos \theta=\gamma_{s}-\gamma_{s t}
$$

where $\theta$ is the contact angle and $S, L$, and $V$ stand for solid, liquid, and vapor, respectively.

Wetting occurs when the contact angle of a liquid front on a solid surface is 
below $90^{\circ}$. According to Young's equation, in this case, the surface energy of the solid is lower when covered with the liquid instead of having direct contact with the gas or vapor phase. The penetration of water or other fluids into paper takes place by capillary flow into effective capillaries. The basic equation for capillary flow developed by Lucas and modified by Washburn is often used to analyse the penetration of liquid into paper. The Lucas-Washburn equation is

$$
\frac{d h}{d t}=r \gamma_{L F} \frac{\cos \theta}{4 \pi h}
$$

where, $t$ is time,

$h$ is the penetration depth of water into the capillary,

$r$ is the capillary radius,

$\gamma_{L V}$ is the liquid surface tension,

$\eta$ is the viscosity of the wetting liquid,

$\theta$ is the contact angle between the liquid and the capillary wall.

Coating and sizing provide paper with resistance to penetration of aqueous solutions. Coating fills the cavities and covers the base paper surface, which decreases the water absorption. Two basic methods of sizing are available: surface sizing and internal sizing. Surface sizing typically utilizes starch particles to fill in the surface voids in the sheet, reducing the pore radius and therefore the rate of liquid 
penetration. Internal sizing utilizes rosin or other chemicals to reduce the rate of water penetration by affecting the contact angle. Rosin is a complex mixture of resinous substances occurring in the natural oleo-resin of softwood.

In coating and offset or lithographic printing processes, the water uptake by paper occurs in a few milliseconds, and the properties of paper such as porosity, roughness, local formation, have significant effects on the paper wetting. For example, in offset printing, it is important to have the water uptake of the paper as small as possible since absorbed water influences the tension in the paper and may result in distortions in the fiber network [21].

\subsubsection{WATER-INDUCED DISTORTIONS}

\subsubsection{INTRODUCTION}

Compared to local small-scale distortions, the phenomena of large-scale distortions are well understood. In the following sections, the large-scale distortions of paper due to moisture changes are reviewed in terms of hygroexpansion, curl, cockle, and sheet roughening as well. This review provides a basis for the understanding of local distortions caused by localized rewerting since the fundamental factors affecting the large-scale and local small-scale distortions could 
be the same or relevant.

\subsubsection{HYGROEXPANSION}

The hygroexpansivity of paper comes from the swelling or contraction of the fibers of which the paper is composed when their moisture content changes. Paper expands when the dimensional changes of fibers transfer to the dimensions of the macroscopic network. Numerous works on hygroexpansity of paper can be found in the literature, and excellent reviews of these studies were given by Gary [3] and Uesaka [40.

Gary [3] concluded that the swelling of the fiber is markedly anisotropic. The main bulk of the fiber wall, the S2 layer, consists of microfibrils which are oriented essentially in a direction parallel to the axis of the fiber. Most of the dimensional change takes place perpendicular to the fiber. Over the range of $\mathrm{RH}$ from $0 \%-100 \%$ at room temperature, the cellulosic fibrils expand approximately $l \%$ in the longitudinal direction of the fibrils and approximately $20 \%$ in the transverse direction. As a result, the dimensional changes of paper are controlled by the transverse expansion or shrinkage of fibers, which is transmitted to the whole network through fiber-to-fiber bonds, while the longitudinal stiffness of fibers resists such transverse changes. 
Nanri and Uesaka [24] investigated the dimensional instability of mechanical pulps including stone groundwood (SGM), thermo-mechanical pulp (TMP), and chemi-thermo-mechanical pulp (CTMP) in terms of drying shrinkage and hygroexpansivity, and experimental results were compared with chemical pulps as reference. Their conclusions were as follows: Mechanical pulps have lower drying shrinkage than chemical pulps, and the lower shrinkage is due to the lower waterretention value (lower swelling ability) of the mechanical pulps; the effect of drying restraint on hygroexpansivity is lower for the mechanical pulps because of their lower drying shrinkage.

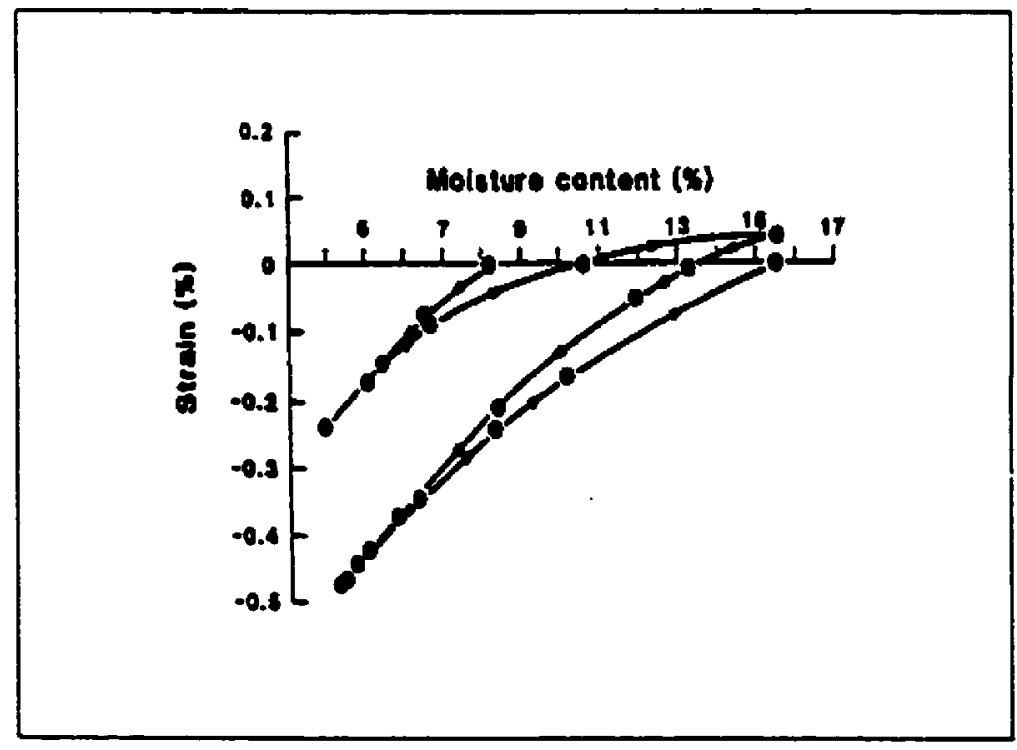

Figure 2.3. Hygroexpansional behavior of paper under cyclic humidity conditions [40] 
An important aspect of hygroexpansivity is hysteresis, the phenomenon of "history-dependence". When paper is exposed to cyclic humidity changes, it expands and shrinks according to its moisture change, but the process is not reversible, and in particular after the first exposure to high humidity it shows a large irreversible shrinkage as Figure 2.3 indicates. Generally speaking, the longer the history of rewetting and drying cycles, the lower the reswelling of fibers [34]. Thus, hysteresis is associated with the drying and rewetting of fibers and of the paper made from these fibers, and is also significant in other dimensional distortions such as curl, waviness, and cockle.

\subsubsection{CURL}

Curl is caused by the uneven expansion or contraction of the two sides of a sheet when it absorbs or loses water $[3,8,19]$. Curling tendency is strongly influenced by sheet two-sidedness. It is also aggravated by the existence of uneven stresses and strains between the top and bottom of a sheet. Generally, there are two types of curl, inherent curl which shows up immediately after the paper is first sheeted, and moisture curl which is due to the tendency of paper to come to an equilibrium moisture content with the relative humidity of its surrounding atmosphere.

There are several known causes of inherent curl in paper. For example, the 
wire side and felt side of a sheet exhibit different degrees of fiber orientation, with wire side fibers tending to be more aligned in the machine direction than felt side fibers. This leads to built-in stresses in the wire side during drying since it has greater tendency to shrink than the felt side. As soon as the paper is sheeted, it curls with the edge bending toward the wire side and the axis of curl parallel to the machine direction. This is a most common type of inherent curl.

Moisture curl occurs when paper is required to come to equilibrium with new atmospheric moisture conditions and the two sides of the paper react differently, producing a differential expansion or contraction. It can arise from two-sidedness, uneven finishing, or uneven absorption of wetting liquid by the two sides of the sheet. Paper coated on one side is an extreme example of paper whose sides show very different moisture absorption; the uncoated side expands while the coated side remains relatively stable when exposed to a higher relative humidity. If the two sides of a sheet have different stresses established in them during drying, they will expand to different degrees in a humid environment, producing a moisture curl.

Curl problems are still troublesome to the papermaker and end user. For example, in developing photosensitive papers; in pasting or applying adhesive to one side of papers and in the printing of the paper, especially with the offset process. More work needs to be done to fully understand curling. 


\subsubsection{COCKLE}

Because of localized nonuniformity of paper, the rate of drying differs from one point to another and the "dried-in" strains will be built into these areas at different stages of drying. The general effect of this variability must be a differential amount of shrinkage from one area to another in the sheet. This must then result in localized outof-plane distortions in the paper termed cockles, and the behavior of the paper is termed cockling $[3,8,31]$. Figure 2.4 is an example of cockle.

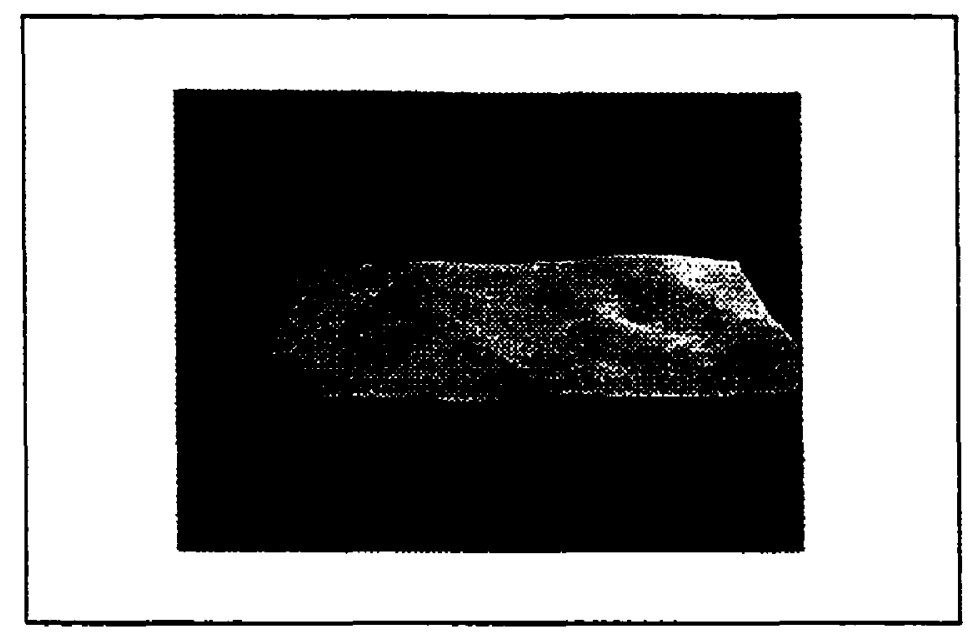

Figure 2.4. Cockles [3I]

Cockle is mostly evident in light to medium weight paper grades. The high bending stiffness of heavier papers prevents cockling. Cockling usually occurs when the moisture content of paper decreases on the paper machine, in converting, or in end use. Common places where cockling appears are the paper machine dryer section and 
electrophotographic printers and copiers. Cockling may also be the result of printing when the moisture content of paper increases. Rewetting and subsequent drying of paper always causes cockling.

Structural unevenness is a necessary requirement for cockling. The most important factors are local grammage, fiber orientation, and two-sidedness of paper. High hygroexpansivity or high hygrocontraction is another necessary requirement for cockling. Bending stiffness determines the ampiitude and size of cockles.

The cockles coincide with the initially less moist areas, with initially more moist areas remaining flat. The explanation appears to lie in the fact that the initial drier areas contract earlier in the first stages of drying and thereby tend to shrink earlier, producing tension at their boundaries that hampers further shrinkage. Other parts of the sheet shrink, as a whole, toward the center upon further drying. The areas already dried cannot shrink any further. Thus, with further contraction of the rest of the sheet, these areas become warped out of the plane of the sheet and form cockles.

\subsubsection{FIBER RISING AND SHEET ROUGHENING}

Fiber rising, a problem that can occur in all processes in which paper is exposed to water, may lead to irreversible changes in the surface structure of paper. In 
printing processes such as inkjet and offset printing, when paper comes into contact with water-based ink or fountain solution, fiber rising may occur. Fiber rising reduces the gloss in the printed image area due to the roughnening of the paper surface.

Hoc [16] developed a laboratory method to determine the fiber rising tendency of papers. The experimental apparatus of this method consisted of three units: moistening, drying, and image anaiysis. The sample was first moistened with water. Then it was dried with hot air and drawn over a roller of small curvature. The fibers lifted from the paper surface were recorded by a video camera linked to a computer. Different grades of paper containing mechanical pulp were investigated using this method, and the measurements agreed well with commercial printing results.

Hoc's method is creative and practical. However, during measurement, the sample needs to be bent through an angle of $90^{\circ}$ over the roller. This may lead to distortion in the paper structure.

Beland et al. [22] developed an image analysis method to quantify the raised fibers on the paper surface. In their experiment, a paper specimen was fixed onto a glass slide using double-sided tape, and then placed on the sample table. Two optical fiber lamps were used to produce low-angle, double-beam illumination to highlight the fibers rising from the paper surface. As a result, the fiber segments appeared 
brighter than their surroundings so that they could be recorded by a video camera, and the number of raised fibers was calculated by analyzing the video image. By means of this method, lightweight coated papers (LWC) and filled supercalendered papers were investigated. The raised fiber index was measured for each sample both before and after water application. The change in gloss was proportional to the change in the raised fiber index.

The use of this technique can avoid any disruption of the paper structure, which may occur with the previous method developed by Beland et al., since the paper surface is flat when analyzed. In addition, the experimental procedure is not complicated. This method seems applicable. However, it was introduced in 1993, and so far, no more information about it can be found in the literature.

Surface roughness refers to the uneven surface of paper or board, which is one of the important paper properties introduced in Section 2.1.3. The increase in roughness affects the optical properties such as gloss, the absorption of ink, and the amount of coating necessary.

Forseth and Helle [23] investigated the effect of wetting on the roughness of different wood-containing base papers: uncalendered and precalendered; hydrophobized and nonhydrophobized. A laboratory blade coater was used to wet the samples. The authors reported that, after wetting, no change in roughness was 
observed on the uncalendered base papers, but there was a significant change for the precalendered base papers. As well, it was reported that the water-induced roughening of the precalendered base paper could be reduced by increasing its hydrophobicity. This research revealed some fundamental factors of water-induced roughening. However, in this study, the roughness measurement was carried out by using a Parker Printer Surface Tester and a mechanical stylus profilometer. Both of these devices may damage the paper surface during measurement.

Using an environmental scanning electron microscope ESEM, Forsberg and Lepoutre [25] investigated a commercial light weight coated (LWC) paper. Images were taken before and after exposure to water. The images clearly showed the roughening caused by the rising of single fibers under the coating, in the presence of water.

Aspler and Beland [6] analyzed the reasons for fiber rising. It was concluded that fiber rising is caused by bond breakage, stress relaxation, and fiber swelling in the presence of water. In addition, the following factors increase fiber rising: more water applied to paper and greater water absorbency; more heat applied to paper, longer contact time between water and paper, more mechanical pulp content. Mechanical fiber is considered to be one of the most important factors in fiber rising and sheet roughening. If more chemical pulp is used, there is less fiber rising. The 
bonds of chemical fibers are stronger, and the strong structure can resist the changes in fiber network.

\subsection{MEASUREMENT OF ROUGHNESS}

\subsubsection{OPTICAL SURFACE PROFILOMETRY}

\subsubsection{CONFOCAL LASER SCANNING MICROSCOPY}

The first patent describing confocal microscopy was registered by Minsky in 1957. While trying to work out the wiring diagram of solidly packed cells in the tissue of central nervous system, he faced a major probiem that he had no way to visualize a three dimensional region of the sample, and it is necessary for him to have a method to control light scattering. He found that the way to avoid all scattered light was to never allow any unnecessary light to enter in the first place [41].

Confocal microscopy uses pinholes to generate a point source and to limit the light entering the detector to only that reflected from the focal plane. Thus, light originating from outside the focal plane is not reflected. The ability to obtain distinct optical sections through the object is the main advantage of confocal microscopy over conventional microscopy. Figure 2.5 illustrates the confocal principle. A laser 
provides an intense and stable light source. At the light source, a pinhole of fixed size is used to obtain point source illumination. The light passing through the objective and reflected from the object is directed at the detector by a beam splitter. A variablesize pinhole in front of the detector allows only reflected light from the focal spot on the object to reach the detector. A point-to-point image can be obtained by scanning the beam over a specimen [29].

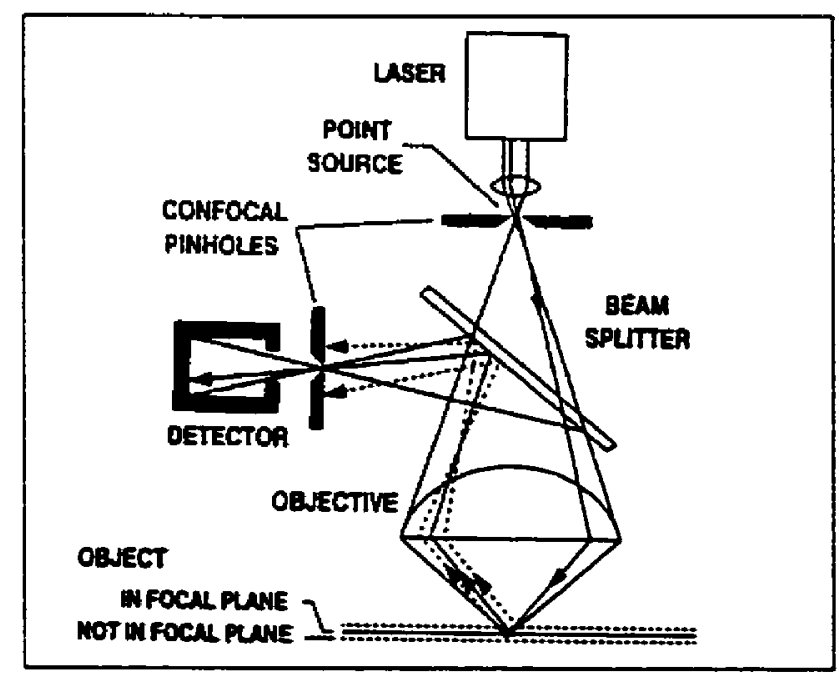

Figure 2.5. Confocal principle [29]

Usually, cofocal microscopy is used in biology and medicine, as well as material sciences. Applications in pulp and paper are also becoming numerous, ranging from investigations of fiber properties to paper surface characteristics $[42,43]$ paper roughness as well as topographical maps can be obtained by means of cofocal 
laser scanning microscopy [29].

The limitation of cofocal laser scanning microscopy is its relatively low resolution. The resolution of cofocal laser scanning microscopy depends on the wavelength of the light and the objective used. In practice, many other factors, such as pinhole size, image format, and depth of the focal plane within the object, are involved. For example, the scanning depth is limited, which is dependent on the opening of the detector pinhole. The pinhole is an important component for determining both the in-plane and out-of-plane resolutions. The more open the pinhole, the thicker the focal plane. When the pinhole is fully open, the image resembles that obtained with a conventional light microscope.

\subsubsection{WYKO OPTICAL SURFACE PROFILOMETER}

The WYKO NT two technologies to measure a wide range of surface heights $[44,45]$. With this system, phase-shifting interferometry (PSI) mode is used to measure smooth surfaces, while vertical-scanning interferometry (VSI) is employed to measure rough surface and step changes in surface height. 
The WYKO ${ }^{\text {tw }}$ NT-2000 system consists two parts: one is the microscope unit and another is the computer (see Figure 2.6). The microscope unit includes an interferometric microscope and its related hardware. The interferometric microscope is the apparatus that is used to scan samples. Included with the unit are a multiple magnification detector and an auto-filter assembly. The computer unit consists of a PC-compatible computer supplied with WYKO Version32 ${ }^{\text {TM }}$ software package running under Microsoft Windows.

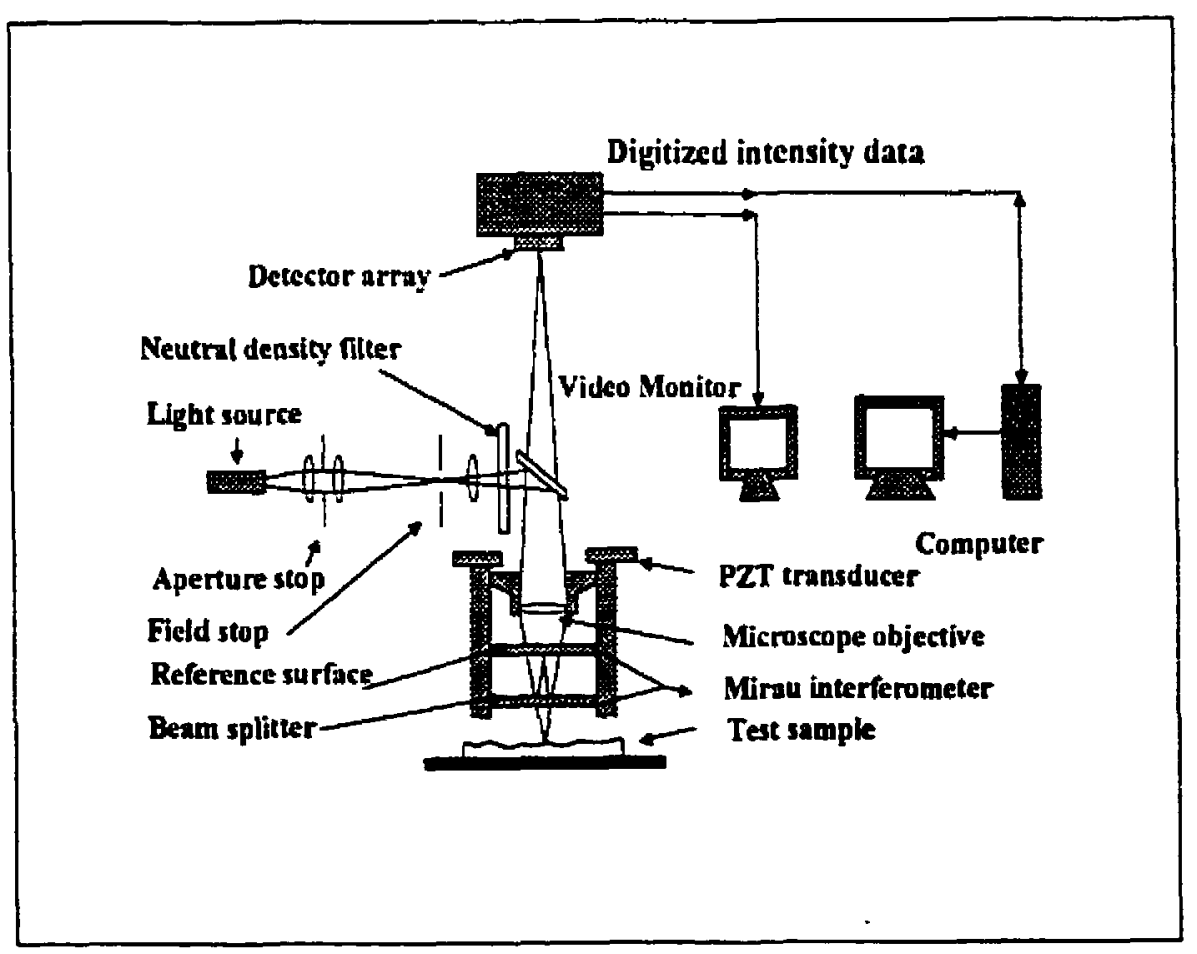

Figure 2.6. WYKO ${ }^{\mathrm{TM}}$ NT-2000 system 
The principle behind WYKO optical surface profilometery is light interference. In Vertical Scanning Interferometry (VSI) mode, during measurement, as Figure 2.6 shows, a white-light beam passes through the microscope objective to the sample. A beam splitter reflects half of the incident beam to the reference surface. The beams reflected from the sample and the reference surface recombine at the beam slitter to form interference fringes. The reference arm containing the interferometric objective moves downward vertically to scan the sample surface so that each point on the surface produces an interference signal. A linearized piezoelectric transducer precisely controls the motion. At evenly spaced internals during the scanning, "frames" of interference data imaged by the video camera are captured and processed. Using a series of advanced computer algorithms, the system precisely locates the peak of the interference signal for each point on the surface and processes them.

WYKO can samples at ambient atmosphere; compared with confocal laser scanning microscopy it has the advantages of high resolutions, both in-plane and outof-plane. Its software provides versatile surface statistical parameters and topgraphic information including 2D and 3D maps for the analysis of paper surface and structure. WYKO was evaluated and used in this study for the measure of roughness and the investigation of local distortions caused by local rewetting. 


\subsubsection{TRADITIONAL METHODS}

\subsubsection{AIR FLOW METHODS}

In practice, paper roughness is usually measured with an air flow method. The common instruments of this type are the Bekk, Bendtsen, Sheffield, and Parker Print Surf (PPS). These instruments either measure the time required for a given volume of air to flow between the paper surface and an optically flat surface pressed against it, or measure the flow rate of the same air. A rougher surface will have greater air flowing through the voids and depressions of the paper.

Bekk smoothness $(\mathrm{sec} / 10 \mathrm{ml})$ measures the air flow between a polished glass and a paper surface under defined conditions of surface geometry, air pressure differences and applied pressure $(98 \mathrm{kPa})$ between the surface. In contrast with the other three methods, Bekk reading increases with the increasing smoothness. Bendtsen roughness $(\mathrm{mL} / \mathrm{min})$ measures the air flow between a narrow $(150 \mu \mathrm{m})$ annular land of diameter $32 \mathrm{~mm}$ and a contacting paper surface compressed under 98 $\mathrm{kPa}$, using a specific pressure difference between the air within the land and the outer atmosphere. Sheffield smoothness uses two concentric annuli between which the air pressure is higher than that of the atmosphere in the outer and inner zones. Table 2.1 shows the characteristics of the different air flow roughness measurement methods. 
Table 2.1. Air flow roughness measurement methods and their characteristics.

\begin{tabular}{|c|c|c|c|c|c|}
\hline Instrument & $\begin{array}{c}\text { Pressure on } \\
\text { paper surface } \\
\text { F/ contact area }\end{array}$ & $\begin{array}{c}\text { External radius } \\
\text { of } \\
\text { contact } \mathrm{R} \mathrm{mm}\end{array}$ & $\mathrm{b} \mathrm{mm}$ & $\begin{array}{c}\text { Air pressure } \\
\text { difference } \\
\Delta \mathrm{P} \mathrm{kPa}\end{array}$ & Unit \\
\hline Bekk & $98 \mathrm{kPa}$ & 18.7 & 12.8 & 49.3 & $\mathrm{sec} / 10 \mathrm{ml}$ \\
\hline Bendtsen & $98 \mathrm{kPa}$ & 16.15 & 0.15 & 1.47 & $\mathrm{~m} / \mathrm{min}$ \\
\hline Sheffield & $100 \mathrm{kPa}$ & $17.2 / 23.5$ & 0.38 & 8 & $\mathrm{~m} / / \mathrm{min}$ \\
\hline PPS & $0.5,1$, or $2 \mathrm{MPa}$ & 15.951 & 0.051 & 6.17 & $\mu \mathrm{m}$ \\
\hline
\end{tabular}

Note: $b$ is the width of the anmilar land, refer to Figure 2.7

The Parker Print Surf (PPS) method is a refinement of Bendtsen. The annular land is further narrowed to $51 \mu \mathrm{m}$ as shown in Figure 2.7 and annular zones about 1.5 $\mathrm{mm}$ wide on each side of the land. The wide inner and outer annuli prevent air leakage from the annular passageways. Selected pressures $(0.5,1.0$, or $2.0 \mathrm{MPa})$ are applied to the paper, simulating printing pressures.

The air flow $Q$ is normally converted to an equivalent gap $G$ in microns between the surface of the narrow annular land and a flat, parallel surface $[27,28]$ :

$$
G=\left(\frac{12 \mu b Q}{L \Delta P}\right)^{1 / 3}
$$


where $\mu$ is the viscosity of air,

$b$ is the radial width of the annulus,

$Q$ is the volume of the air flowing in unit time,

$L$ is the circumference of the annulus,

$\Delta P$ is the pressure drop across metering land.

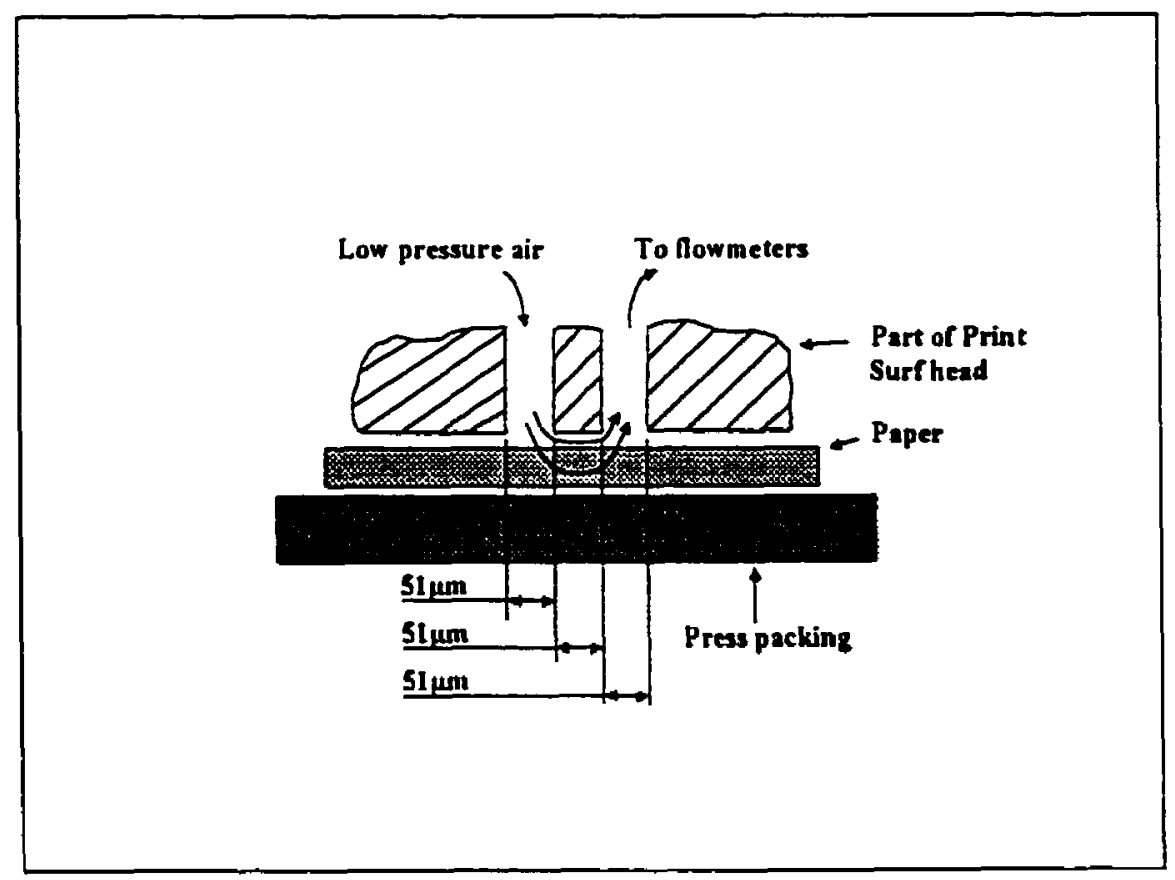

Figure 2.7. Section of part of a Print-Surf head, showing the measuring anmilus in contact with the paper [28]

\subsubsection{MECHANICAL STYLUS PROFILOMETRY}

Mechanical stylus profilometry is a common technique used to characterize 
the surface roughness of materials such as metals. The technique uses a fine, coneshaped diamond stylus applied on the surface with a given load. Measurement of the vertical movement of the diamond stylus occurs while it travels along the surface of a sample. A few publications have discussed the technique to characterize paper and board surfaces.

Kapoor et al. [55] used stylus profilometry to evaluate the printing smoothness of coated papers. The paper roughness profiles and roughness data in terms of root mean square roughness were obtained in their study. Based on the experimental data, the printed papers were ranked and it was found that the ranking results obtained using this method agreed with the conventional test results.

With a stylus profilometer capable of measuring variations less than $\mathrm{l} \mu \mathrm{m}$ in height, MacGregor and Conners [56] conducted measurements of both coated and uncoated papers. Table 2.2 shows the average arithmetic roughness results of the samples.

Table 2.2. Average arithmetic roughness of coated and uncoated papers

\begin{tabular}{|l|ccc|}
\hline & Base paper & Coated paper & Coated paper, printed \\
\hline Wire side, $\mu \mathrm{m}$ & 3.9 & 0.83 & 1.57 \\
Felt side, $\mu \mathrm{m}$ & N/A & 0.16 & 1.94 \\
\hline
\end{tabular}


In order to evaluate a confocal optical scanning profilometer, Wagberg et al. [56] used a mechanical stylus profilometer to measure the roughness of seven printing papers. It was reported that there was good agreement between these two methods, but the confocal optical scanning method yielded a slightly higher roughness than the mechanical stylus profilometry.

It was noted that the mechanical stylus leaves a mark on paper. Murakami et al. [57] admitted that scratches inevitably appear on paper. Wagberg et al. [58] also reported the problem. They observed that the tracks in the coated samples were all visible to the naked eye, but no tracks were detectable in uncoated samples. Enomae et al. [59] utilized SEM to examine the traces left by the stylus when performing stylus profilometry. It was concluded that the stylus marking depends on stylus conditions (i.e., radius of the diamond tip and load of the stylus) as well on the surface hardness of paper. If a track is left, the measurement may be flawed. However, since the stylus travels with a load on both ridges and valleys of the paper surface the measurement may represent the real profile. As well, by careful choice of stylus radius and load conditions for the particular surface, one can ensure there is no permanent deformation. 


\section{CHAPTER THREE}

\section{EVALUATION OF WYKO ${ }^{\text {TM }}$ NT-2000 SYSTEM}

\subsection{INTRODUCTION}

The WYKO ${ }^{\text {TM }}$ NT-2000 system, which is in the laboratories of Photonics Research Ontario (PRO) located in the Physics department of the University of Toronto, is the key facility for this study. Compared with other surface profilometers, WYKO is a non-contact instrument with large scanning depth and high resolution, which can produce highly accurate 2D and 3D maps of a surface under ambient atmosphere.

The WYKO ${ }^{\text {TM }}$ NT-2000 system has been used to characterize the surface textures of many materials such as aluminum, steel, plastics, ceramics, semiconductors, and optical fibers. However, in the literature, there is no information about the application of WYKO surface profilometry to the study of paper surface. Based on its satisfactory performance in other research fields, it was assumed that it would be a useful apparatus for the characterization of paper surface. Considering that samples can be scanned without any damage under natural conditions, WYKO surface profilometry provides an excellent opportunity for the investigation of local distortions that are caused by localized rewetting. Nevertheless, before WYKO was 
applied to the study of the relationship between local distortion and rewetting of paper, it was evaluated by comparing WYKO experimental results of roughness with the data obtained by using traditional methods including Parker-Print Surf (PPS) air flow method and mechanical stylus profilometry.

\subsection{WYKO SURFACE SCANNING PROFILOMETRY}

\subsubsection{EXPERIMENTAL SETUP}

The WYKO ${ }^{\text {TM }}$ NT-2000 system consists of the following hardware components: a Mirau-type interferometeric microscope, a vibration isolation table, a video monitor, and a PC-compatible computer supplied with WYKO's Vision32 ${ }^{\text {Th }}$ software.

The main unit of the WYKO ${ }^{\text {TM }}$ NT-2000 system is the interferometric microscope and its related hardware, as shown in Figure 3.1. The interferometric microscope is the unit that allows one to focus on the sample. The related hardware includes an illumination system, a field of view assembly, a magnification head, and a sample stage. The illuminator contains a white light illumination source. Using WYKO's Vision $32^{\text {TM }}$ software, one can vary the intensity of this light source. The field of view assembly allows one to select various lens tube positions for use in 
aligning the system or focusing on a sample. The interferometric microscope is equipped with one turret, which houses three objectives. The sample stage is located beneath the magnification objective. During the experiments for the investigation of local distortions caused by rewetting, a micrometer stage with a special clamp screwed on was used, which makes it possible to relocate the sample to its original position after being taken off.

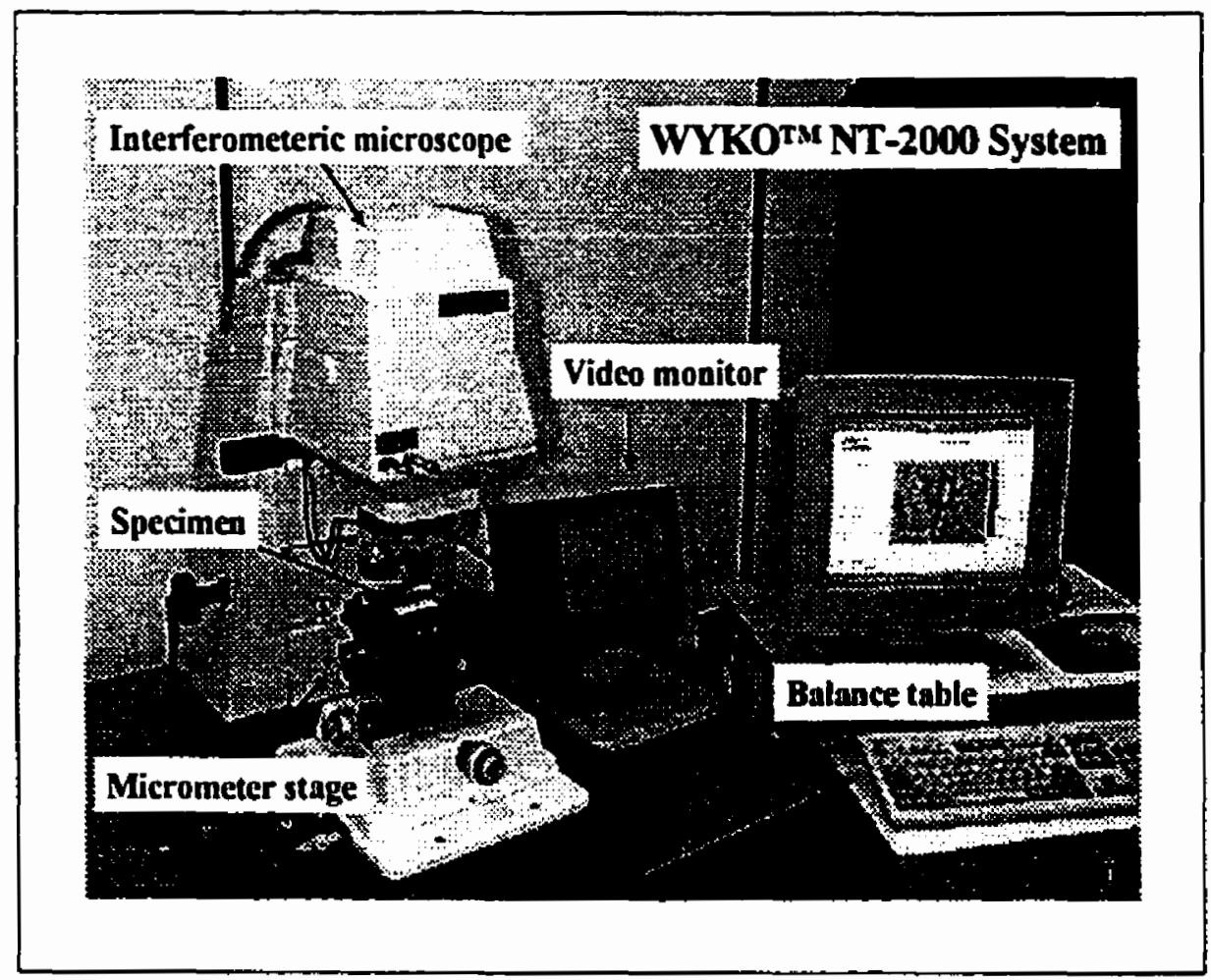

Figure 3.1. Experimental sethp (WYKO ${ }^{\mathrm{Tu}}$ NT-2000 system) 
The WYKO interferometeric microscope unit is sensitive to vibrations that can cause the fringe pattern to shake or wave. These vibrations will reduce the accuracy and repeatability of the measurements. Therefore, the microscope unit is placed on an air isolation table specially designed to damp out the vibrational frequencies.

The video monitor provides a real-time image of the sample as seen through the magnification objective. During alignment and focusing, the reflected images of the sample can be viewed in the video monitor screen.

The WYKO ${ }^{\text {TM }}$ NT-2000 computer system consists of a PC-compatible computer supplied with WYKO's Vision32 ${ }^{\text {TM }}$ software package nunning under Microsoft Windows. WYKO's Vision $32^{\text {TM }}$ software works with WYKO ${ }^{\text {TM }}$ NT-2000 system to provide analysis capabilities, and contains pre-set configuration files for the operations.

To measure a sample, first of all, one should selected a suitable magnification and relevant operating parameters including scanning depth, scanning speed, and light intensity. Then, focus on the sample by adjusting the distance between its surface and the objective. Finally, click the icon of "image acquire" to scan the sample, and a digital image of a local region on the sample surface will be obtained once the scanning is completed. This image can be analyzed using the Vision $32^{\text {tM }}$ software, 
and statistical surface parameters such as arithmetic average roughness, root mean square roughness, and skewness are available.

\subsubsection{PERFORMANCE OF WYKO}

The performance of one system depends to some extent on the measurement technique. To obtain optimum system performance, the operators should always use a well-calibrated system and consistent measurement techniques; as well, make sure that environmental noise is minimized by setting up the system as decribed in the operator's guide. The performance of the WYKO ${ }^{\text {TM }}$ NT-2000 system can be described in terms of range, resolution, and accuracy.

Range refers to the greatest vertical distance a profilometer can accurately measure. For WYKO interferometric profilometer, the range of depth that VSI (vertical-scanning interferometry) mode can profile is limited only by the range of the PZT (piezoelectric transducer) used to perform the translation through focus; the system used here could scan up to $500 \mu \mathrm{m}$.

Resolution refers to the smallest distance that the WYKO profilometer can accurately measure. It can be specified for either in-plane or out-of-plane resolutions. If the application deals with surface height and roughness, out-of-plane resolution 
may be critical. If the application deals with surface height and out-of-plane measurements of surface features, both in-plane and out-of-plane resolutions may be critical. In-plane resolution is a function of the magnification of the objective and the array size of the detector. For WYKO ${ }^{\text {TM }}$ NT-2000 system, various magnifications can be chosen while the array size of the detector is a constant value of $736 \mathrm{X} 480$. Table 3.1 details the magnifications, field of view, and the in-plane resolutions. The vertical resolution, for VSI mode, is less than I $\mathrm{nm}$.

Table 3.1. Magnifications, field of view, and in-plane resolutions of the WYKO ${ }^{r M} N T-2000$ system.

\begin{tabular}{|c|c|c|}
\hline Magnification & $\begin{array}{c}\text { Field of view } \\
\mu \mathrm{m}\end{array}$ & $\begin{array}{c}\text { In-plane resolution } \\
\mu \mathrm{m}\end{array}$ \\
\hline 5 & $1200 \times 900$ & $1.63 \times 1.88$ \\
10 & $610 \times 460$ & $0.83 \times 0.96$ \\
20 & $300 \times 230$ & $0.41 \times 0.48$ \\
25 & $240 \times 180$ & $0.33 \times 0.38$ \\
40 & $150 \times 110$ & $0.20 \times 0.23$ \\
50 & $120 \times 90$ & $0.16 \times 0.19$ \\
100 & $60 \times 40$ & $0.08 \times 0.08$ \\
\hline
\end{tabular}

Accuracy reters to how closely a measured value matches the true value. It is determined relative to a known, traceable standard. The accuracy of the system can be 
checked by measuring a step height standard and comparing the result to the true value. The WYKO ${ }^{\mathrm{m}}$ NT-2000 system is calibrated weekly by the Photonics Research Ontario (PRO) technicians.

\subsubsection{MEASUREMENT OF ROUGHNESS}

Seven samples including coated and uncoated papers were measured. The samples were MWC-WO, LWC-WO, LWC-RG, SC, Newsprint, TMP No.1, and TMP No.2. Table 3.2 includes these samples as well as two sized copy papers, which was used for the investigation of local distortion caused by local rewetting.

The specimens of papers were fixed onto glass slides of $76 \times 26 \mathrm{~mm}$ using double-sized tape to ensure the surface of paper remained flat. For each sample, six measurements were conducted at different locations on the same specimen. The scanning depth for every measurement was $100 \mu \mathrm{m}$; the magnification was 10 . The corresponding scanning field (field of view) was $603 \times 459 \mu \mathrm{m}$. The in-plane resolutions were $0.83 \times 0.96 \mu \mathrm{m}$, and the out-of plane resolution was less than $1 \mu \mathrm{m}$. The image analysis software WYKO vision $32^{\mathrm{TM}}$ was used to quantify the images in terms of arithmetic average roughness $\left(R_{a}\right)$. The experimental results are presented in Section 3.4. 
Table 3.2. Paper samples and their suppliers

\begin{tabular}{|l|l|c|}
\hline \multicolumn{1}{|c|}{ Sample } & \multicolumn{1}{|c|}{ Description } & Suppliers \\
\hline MWC-WO & Medium weight coated offset paper & UPM Kymmene \\
LWC-WO & Light weight coated offset paper & UPM Kymmene \\
LWC-RG & Light weight coated rotogravure paper & UPM Kymmene \\
SC & Supercalendered paper & UPM Kymmene \\
News & Newsprint & UPM Kymmene \\
TMP No.1 & Thermomechanical pulp No.1 & Abitibi Consolidated \\
TMP No.2 & Thermomechanical pulp No.2 & Abitibi Consolidated \\
SS & Surface sized copy paper & Domtar \\
IS & Internally sized copy paper & Domtar \\
\hline
\end{tabular}

\subsection{MECHANICAL STYLUS PROFILOMETRY}

\subsubsection{SURTRONIC $3^{+}$PROFLOMETER}

The Surtronic 3+ stylus profilometer is a portable, self-contained instrument for the measurement of surface texture. It characterizes the surface by traversing the sample with a stylus tip that responds to the height variations along a profile. The parameter evaluations and other functions of the instrument are microprocessor 
based. Parameters available for surface texture evaluation are average arithmetical roughness $\left(R_{a}\right)$ and root means square roughness $\left(R_{q}\right)$. The measurement results are displayed on a LCD screen and can be output to a computer for further evaluation. The Surtronic 3+ includes: display-traverse unit and standard pick-up as Figure 3.2 shows.

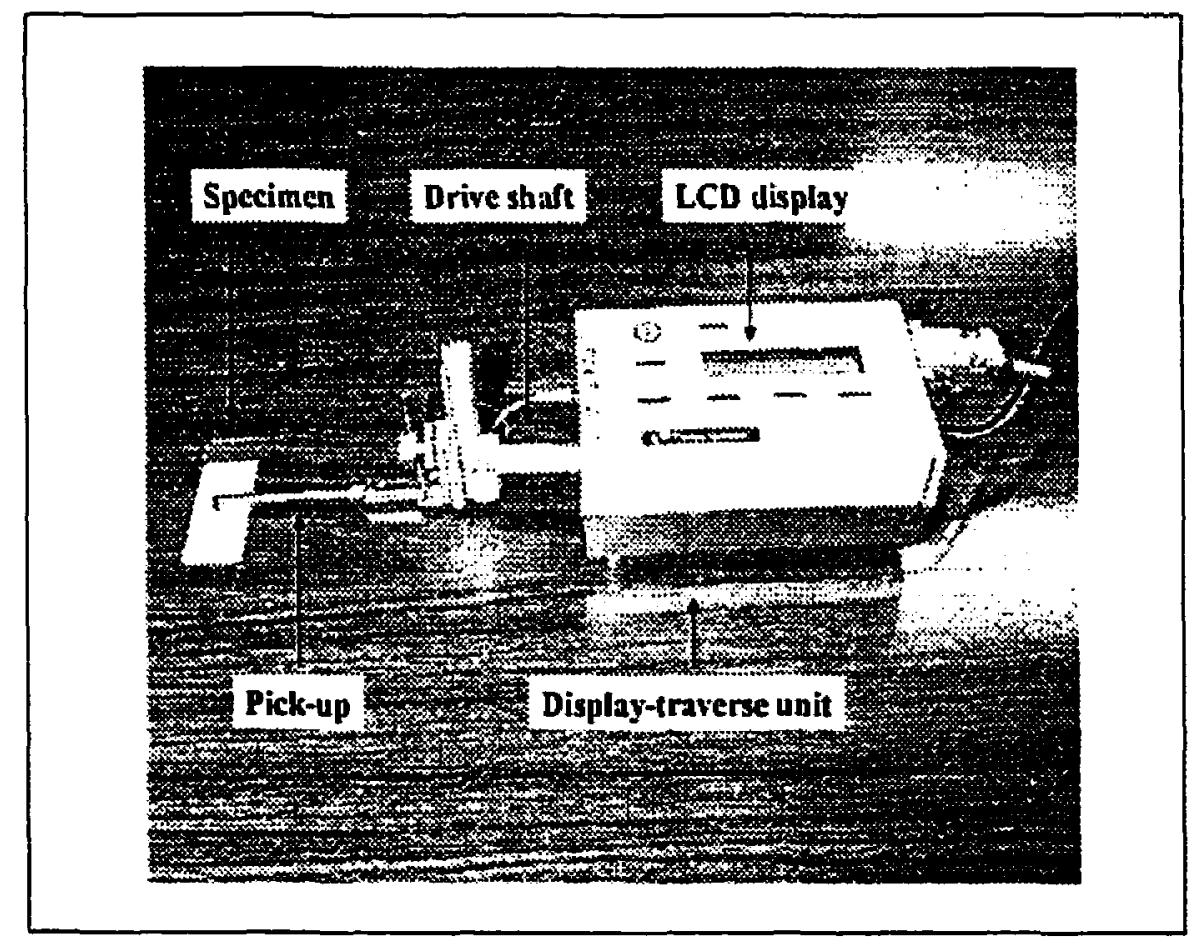

Figure 3.2. The Surtronic 3- stylus profilometer

The top panel of the display-transverse unit carries a membrane type control panel and a liquid crystal display (LCD). The unit houses the electronics for the 
controlling the measurement sequence, computing the measurement data and outputing the results to the display, or to a computer for further analysis. The unit also contains a drive motor, which traverses the pick-up across the surface to be measured. The measuring stroke always starts from the extreme outward position. At the end of the measurement the pick-up returns to this position ready for the next measurement.

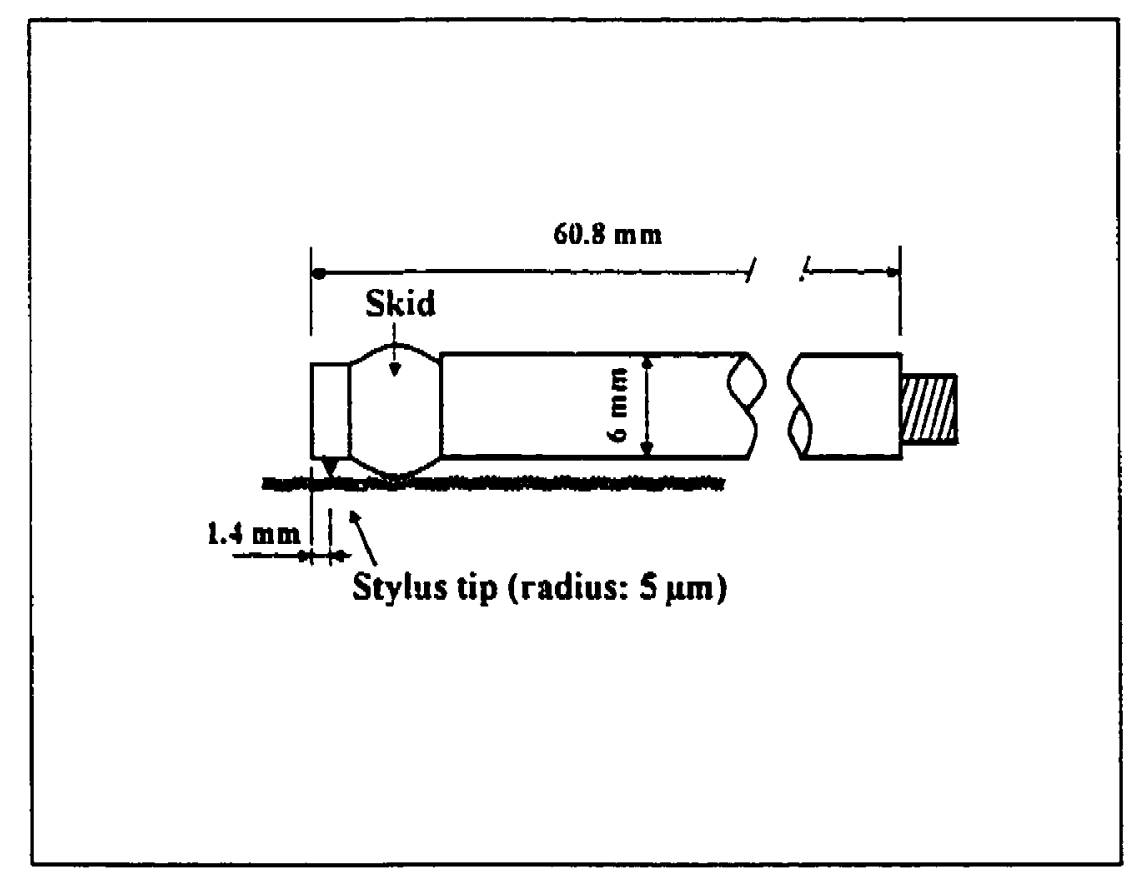

Figure 3.3. The pick-up of the Surtronic 3+stylus profilometer

The pick-up is fastened to the drive shaft and supported on the surface to be measured by a skid (see Figure 3.3), which is a curved support projecting from the underside of the pick-up in the vicinity of the stylus. As the pick-up traverses across the surface, movements of the stylus relative to the skid are detected and converted 
into a proportional electronic signal. The radius of curvature of the skid is much greater than the roughness spacing. This enables it to ride across the surface almost unaffected by the roughness, and provide a datum representing the general form of the surface. The radius of the stylus tip was $5 \mu \mathrm{m}$.

\subsubsection{MEASUREMENT OF ROUGHNESS}

The same samples described in Section 3.2.3 were measured using the Surtronic $3+$ stylus profilometer. Specimens were prepared in the same way as those used for the WYKO. For each sample, one specimen was used, and twenty measurements were conducted on this specimen, 10 times on $C D$ (cross-machine direction) and 10 times on MD (machine direction). For all the measurements, the evaluation length $(\mathrm{Ln})$, i.e., the length over which the values of surface analysis parameters are assessed, was chosen as $8 \mathrm{~mm}$. The in-plane resolution was $0.5 \mu \mathrm{m}$, and the out-of-plane resolution was $10 \mathrm{~nm}$. With the Surtronic $3+$ stylus analysis software, surface parameters were also expressed in terms of arithmetic average roughness $\left(R_{\mathbf{a}}\right)$. The experimental results are tabled in Section 3.4, compared with the roughness results obtained by means of WYKO.

\subsection{EXPERIMENTAL RESULTS AND COMPARISON}


Table 3.3 shows the experimental results of roughness measured using the WYKO surface scanning profilometer and the Surtronic $3+$ stylus plofolometer, as well as the roughness data obtained using conventional Parker-Surf Print air flow method. PPS roughness data were provided by the sample suppliers. The WYKO vision $32^{\text {TM }}$ software provides option of tilt-removal as well as smoothing filter of median pass to eliminate the noise. All of the WYKO images in this study were corrected for mean tilt and smoothed with a median pass filter. Tilt-removal makes the slanted samples flat. The spatial smoothing algorithm uses an array of height data within a window. The program adjusts the height values of center point according to

Table 3.3. Roughness - comparison among WYKO, mechanical profilometry, and PPS

\begin{tabular}{|l|ccc|}
\hline Sample & WYKO, Ra, $\mu \mathrm{m}$ & Surtronic 3+, Ra, $\mu \mathrm{m}$ & PPS, $\mu \mathrm{m}$ \\
\hline TMP No.1 & $4.12 \pm 0.36$ & $3.39 \pm 0.34$ & 3.80 \\
TMP No.2 & $3.17 \pm 0.39$ & $2.81 \pm 0.23$ & 3.14 \\
Newsprint & $3.55 \pm 0.21$ & $3.02 \pm 0.24$ & 3.94 \\
SC & $1.17 \pm 0.11$ & $1.27 \pm 0.10$ & 1.09 \\
LWC-WO & $1.14 \pm 0.16$ & $1.08 \pm 0.09$ & 1.33 \\
LWC-RG & $0.79 \pm 0.12$ & $1.02 \pm 0.12$ & 0.69 \\
MWC-WO & $0.68 \pm 0.05$ & $0.72 \pm 0.07$ & 0.80 \\
\hline
\end{tabular}


the height values of the neighboring data points in the window (array), moves the window to the next location, and repeats the calculation. This is done for the entire data set. For median smoothing filter, the height values in the window are sorted in ascending order, and the median of this sort is stored as the new center height. For the Surtronic $3+$ stylus profilometer, the measuring length was $8 \mathrm{~mm}$; and the inplane resolution was $0.5 \mu \mathrm{m}$.

In this study, for WYKO, $\mathrm{R}_{\mathrm{a}}$ is the arithmetic average of the absolute values of the measured height deviations taken within the scanned evaluation area and measured from the mean surface. The digital approximation for the $3 D R_{a}$ is:

$$
R_{a}=\frac{1}{M N} \sum_{i}^{M} \sum_{j}^{V}\left|Z_{i j}\right|
$$

where $M$ is the number of data points in $\mathrm{X}$ direction of the array,

$N$ is the number of data points in $\mathrm{Y}$ direction of the array, $Z_{i j}$ is the local surface height based on the mean surface.

The roughness data obtained using the Surtronic $3+$ stylus profilometer is $2 \mathrm{D}$ $\mathbf{R}_{\mathfrak{a}}$, which is digitally formulated as: 


$$
R_{a}=\frac{1}{N} \sum_{i}^{N}\left|Z_{i}\right|
$$

where, $N$ is the number of data points within the measure line,

$Z_{i}$ is the local surface height based on the mean line.

As Table 3.3 presents, for both coated and uncoated paper, the agreement between the WYKO $R_{a}$ and the PPS roughness is fairly good. There is also a good agreement between WYKO and the Surtronic 3+ stylus profilometer even though the $\mathbf{R}_{\mathrm{a}}$ measured with mechanical stylus is lower for uncoated samples, except for supercalendered paper (SC). This was probably due to the effect of the skid used as the supporter for the stylus. Especially, for the detection of the details of the deep valley the mechanical stylus presents a limitation since the diameter of the stylus tip is $5 \mu \mathrm{m}$. As well, it is impossible to measure the true top of the paper surface since the load on the stylus will cause an imprint during a measurement [58-60]. On the other hand, since WYKO is an optical surface profilometer there is no any damage to a specimen during measurement; and a beam of light can scan into the valley of the specimen, which represents the true details of the sample surface.

The roughness of paper surface is probably dependent on the measured length or area. The WYKO Version 32+ software allows one to capture a window in an 
image for the analysis of the relevant statistic surface parameters. Figure $\mathbf{3 . 4}$ and $\mathbf{3 . 5}$ present the change in arithmetic average roughness $\left(R_{a}\right)$ over the captured area. For all samples, with an increase of captured area there was a slight increasing tendency for $R_{a}$. On the other hand, the standard deviation of $R_{a}$ decreased with the increase of captured area, which is shown in Figure 3.6 and 3.7. It is evident that samples with more coating or high degree of calendering showed relatively smaller change in roughness or standard deviation of roughness than other samples. Therefore, of the three coated papers, medium weight coated paper (MWC-WO) showed the least change in $\mathrm{R}_{\mathrm{a}}$ and the deviation of $\mathrm{R}_{\mathrm{a}}$. Similarly, supercalendered paper (SC) had a relatively stable in roughness as indicated in Figure $\mathbf{3 . 4}$ and 3.6.

The WYKO ${ }^{\text {TM }}$ NT-2000 system also provides topographic images, which allow one to obtain visual information about the paper surface. Figure 3.8 to 3.9 are typical WYKO images of coated paper and uncoated paper used in this study. In these images, the red color represents the relatively high positions on the paper surface while the blue color stands for the relatively low positions. In Figure 3.8, the coverage of the surface is complete and no fibers of the substrate can be seen on the surface of this MWC-WO sample. And for TMP No. 2 sample as shown in Figure 3.9, the individual fibers are visible and the structure of fibrous networking is obvious. 


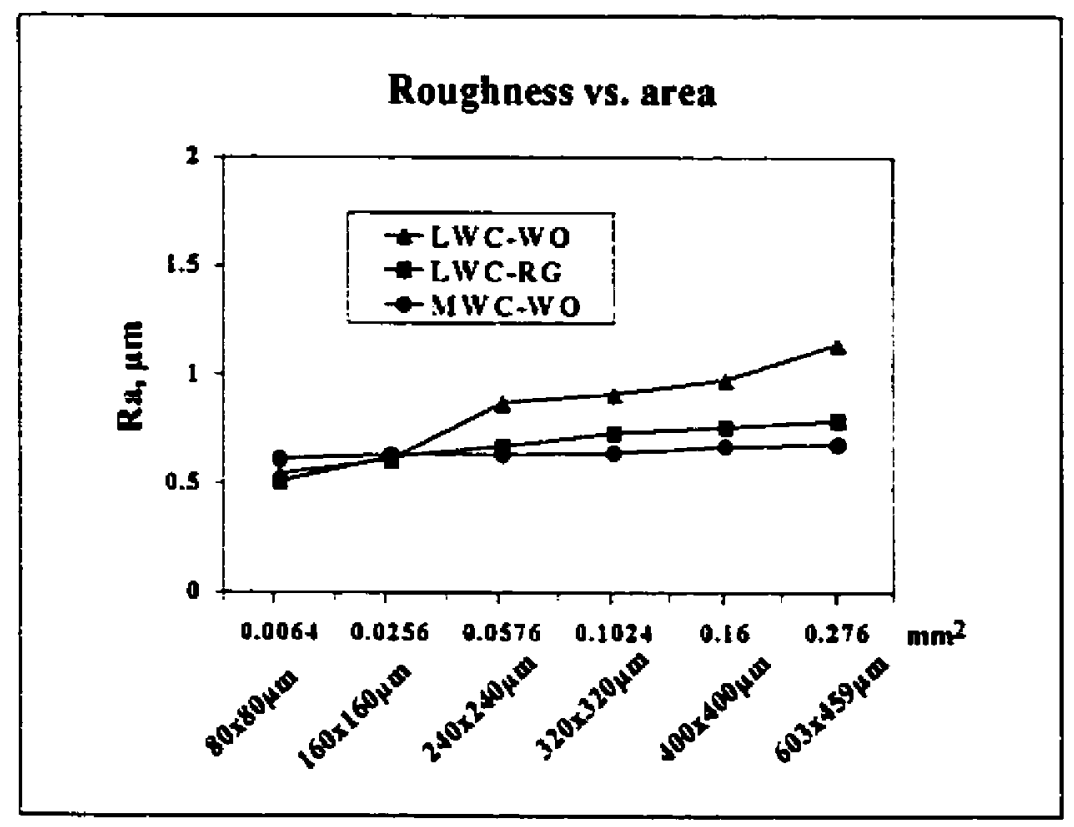

Figure 3.4. The relationship berween roughness of coated paper and measured area

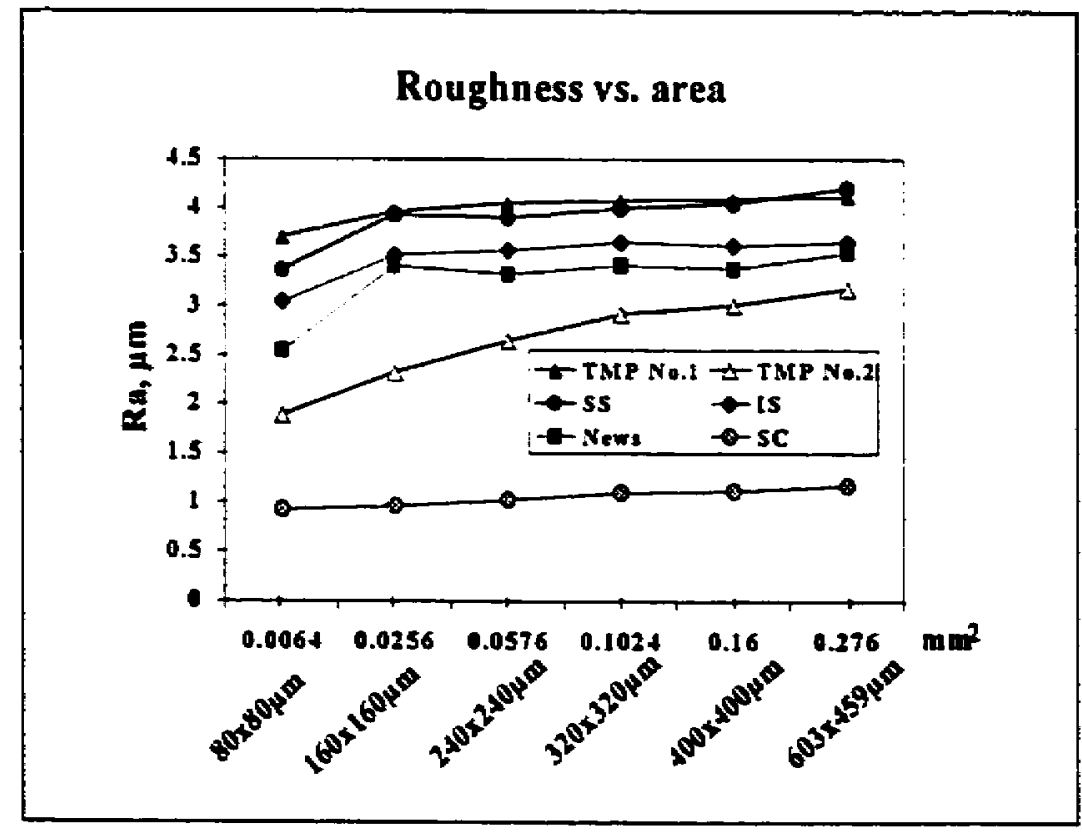

Figure 3.5. The relationship between roughness of uncoated paper and measured area 


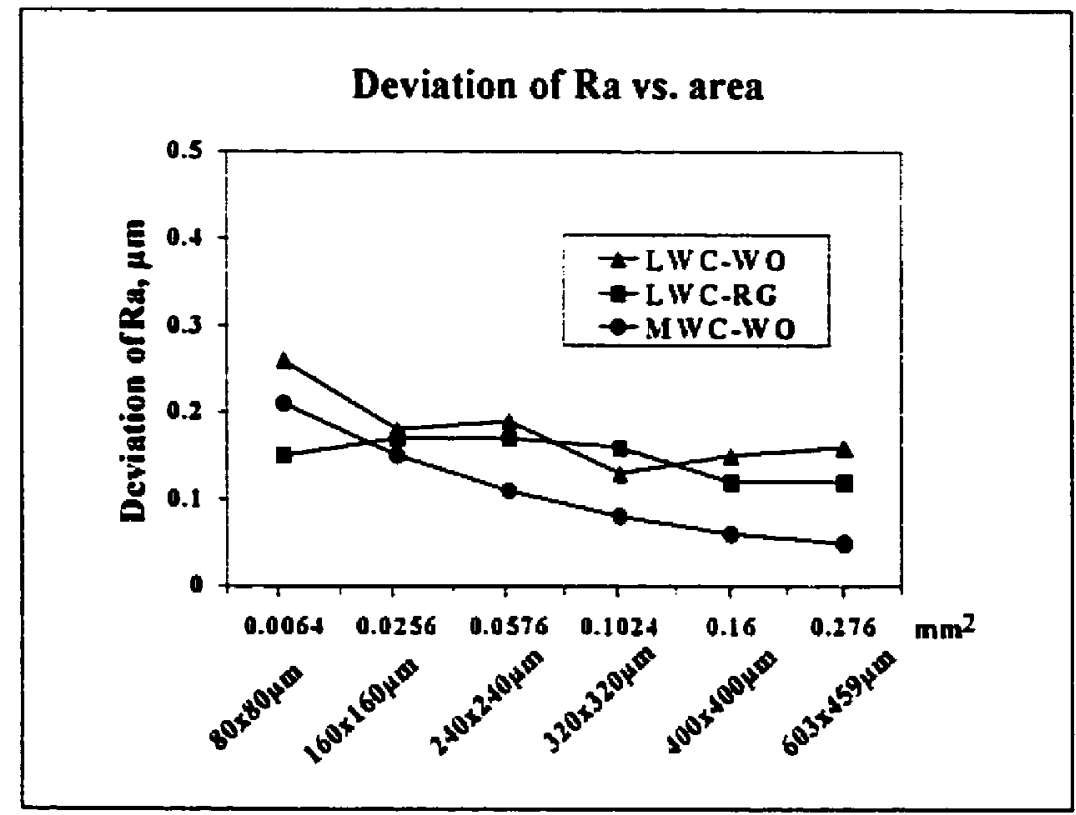

Figure 3.6. The relationship between standard deviation of roughness of coated paper and measured area

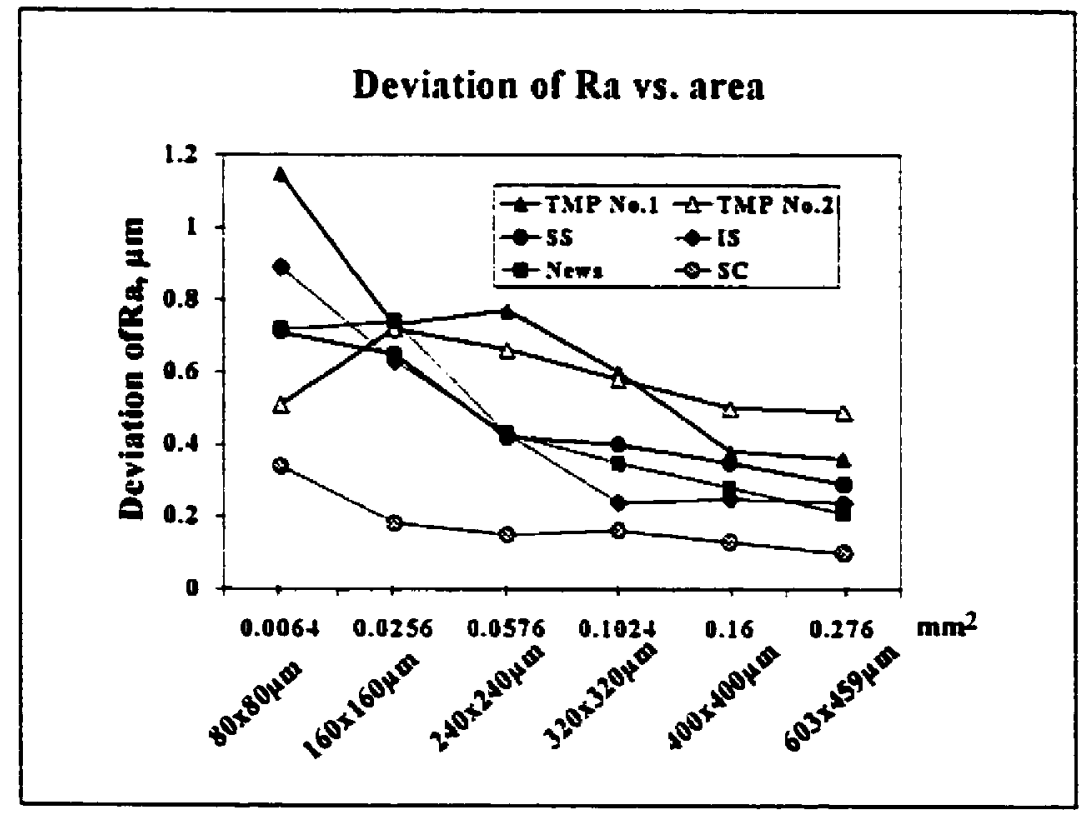

Figure 3.7. The relationship between standard deviation of roughness of uncoated paper and measured area 


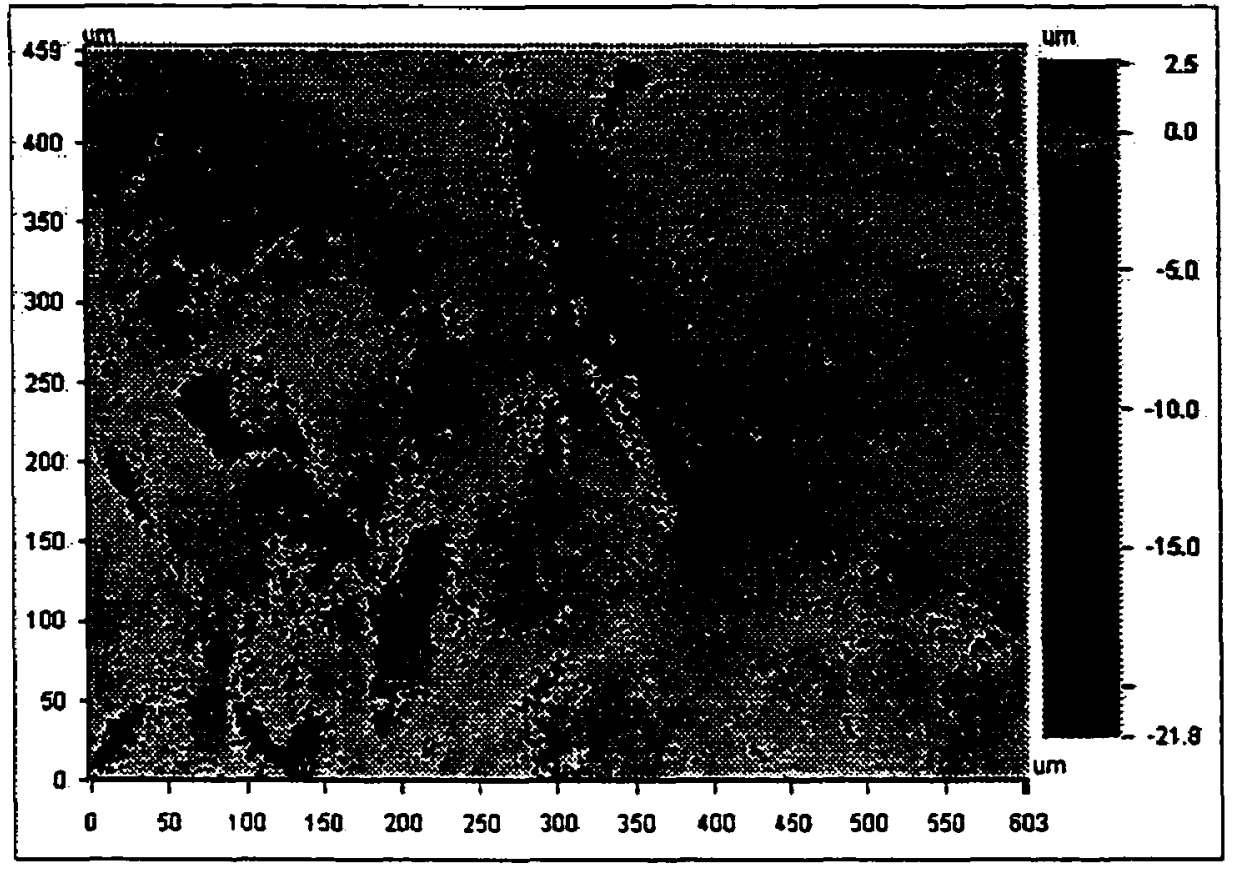

Figure 3.8. Typical WYKO image, MWC-WO

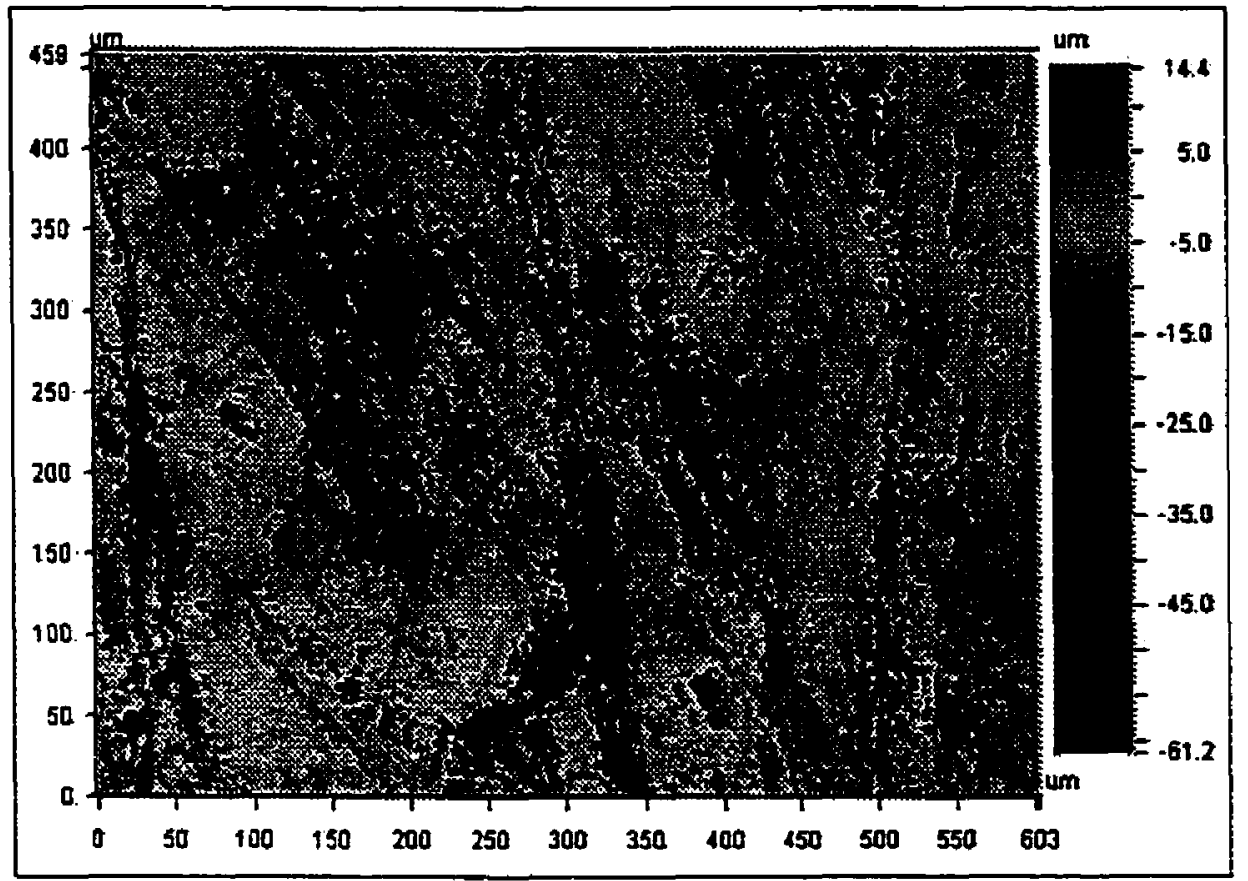

Figure 3.9. Typical WYKO image, IMP No.2 


\subsection{SUMMARY}

Through the measurement of roughness on different grades of paper, WYKO ${ }^{\text {TM }}$ NT-2000 system was assessed. The WYKO experimental results were compared with the data obtained using Parker-Surf Print (PPS) method and mechanical stylus profilometery. The good agreements among WYKO, PPS, and mechanical stylus indicate the WYKO ${ }^{m}$ NT-2000 system is a useful apparatus for the study of paper surface characteristics. In particular, with the advantages of no damage to specimens during measurement, high resolution in both in-plane and out-of-plane directions, and the versatile analysis functions, it was concluded that WYKO optical scanning profilometry is a potential method for the study of local distortions on paper caused by local rewetting. 


\section{CHAPTER FOUR}

\section{DEVELOPMENT OF METHODOLOGY}

\subsection{INTROUCTION}

In order to implement the study on local rewetting and distortion of paper, a local rewetting technology needed to be developed. In this study, an inkjet printer was successfully modified for this purpose. With the modified printer, ink-dyed water or pure water can be used as the wetting liquid, and micro-scale droplets can be produced for local rewetting. The modification of the printer is discussed in this chapter.

The evaluation described in the previous chapter demonstrated that the WYKO $^{\text {tM }}$ NT-2000 system can be used for the investigation of paper surfaces. However, certain obstacles arose during the application of the WYKO to the measurement of the changes in surface height when paper was locally rewetted. The area around the wetted location could not be used as a reference surface to compare the local surface heights before and after rewetting. Because water might diffuse from the wetted area into the surrounding paper, and the neighboring dry area might not really be dry, a smooth surface needed to be introduced into the scanning field as a reference. This reference surface must be stable and not affected by the water 
diffusion. This chapter introduces the development of the WYKO methodology and the testing of the reference surfaces. Finally, based on the method established here, the investigation of local distortions due to local rewetting on different grades of paper was carried out.

\subsection{LOCAL REWETTING TECHNOLOGY}

\subsubsection{PREVIOUS WORK}

A common technology available for micro-scale rewetting is the piezoelectric droplet generator [46-49]. Oliver [49,50] applied this technology to the study of local rewetting on paper. Piezoelectric droplet generators are usually used for inkjet printing.

Inkjet printing uses small drops of ink to form an image on a medium. Two types of inkjet technology exist: continuous flow and drop-on-demand. Continuous flow inkjet printing uses electrostatic acceleration and deflection to select ink drops from a constant flow of ink to form an image. Drop-on-demand inkjet printing comes in two forms, as described in Figure 4.1: piezoelectric and thermal. Piezoelectric uses a mechanical means to eject ink. Thermal inkjet technology uses heat energy to vaporize a thin layer of ink to form a bubble that expels a small drop of ink through 
an orifice or nozzle. As the ink leaves the nozzle head, it creates a vacuum that pulls in fresh ink. The physical components needed to implement thermal inkjet technology are embodied in the inkjet print cartridge. Hewlett-Packard (HP) uses both piezoelectric and thermal inkjet printing technology [51].

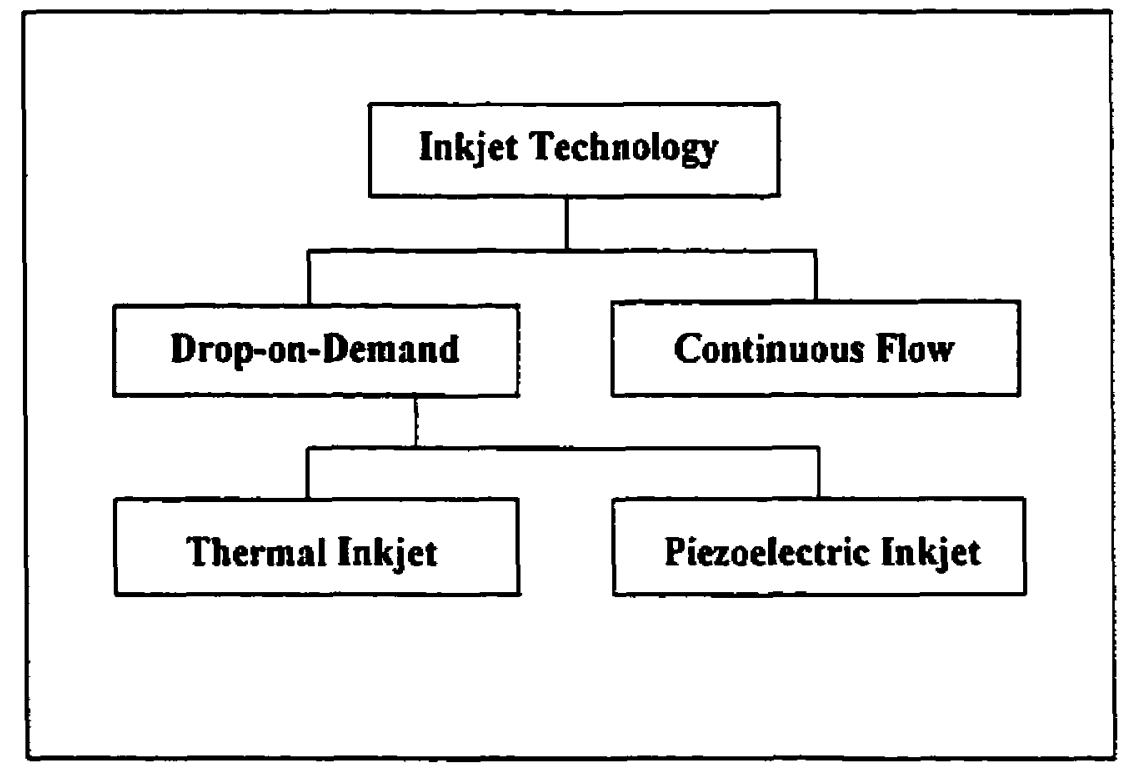

Figure 4.1. Inkjet technology

\subsubsection{MODIFICATION OF AN INKJET PRINTER}

In this research, a HP 520 inkjet printer was modified for the paper sample rewetting. Two HP 51625A cartridges, which match the inkjet HP 520 printer, were 
also modified. As a result, small droplets up to $50 \mu \mathrm{m}$ in diameter can be generated using the modified inkjet printer and cartridges.

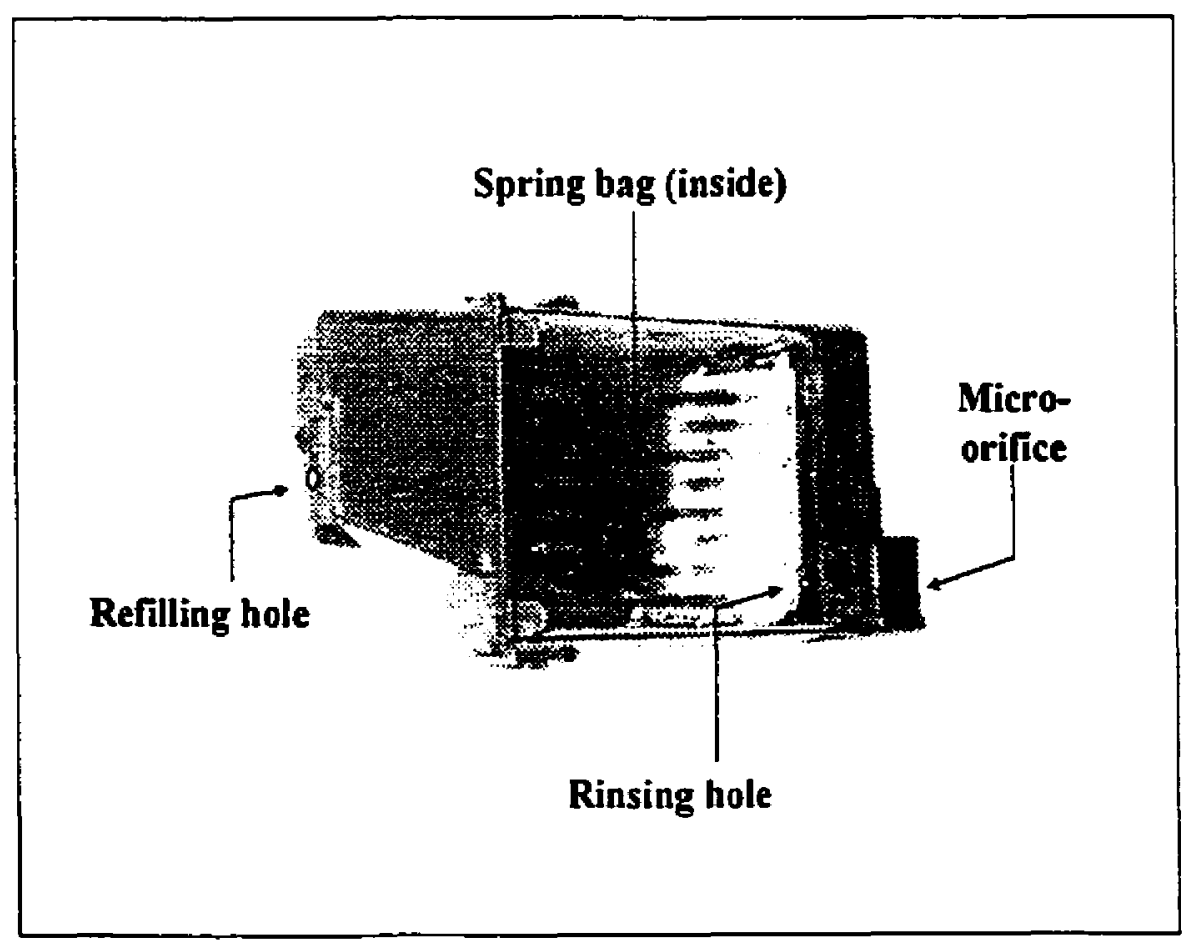

Figure 4.2. A modified ink cartridge with pure water inside

The HP 51625A ink cartridge was a sealed plastic box with an internal spring bag, which develops slight vacuum to retain the ink. The wetting liquids used in this research were ink-dyed water and distilled pure water. Therefore, ink in the cartridge was replaced with ink-dyed water or distillated pure water. Figure 4.2 shows the cartridge, which was refilled with pure water. There is a small hole sealed with a thin 
film located at the top of the HP 51625A ink cartridge. It is designed for ink refilling. In this study, this hole was opened and used for the refilling of ink-dyed or pure water. In addition, a small hole was drilled in one side of the box, which made it easy to withdraw the ink inside and rinse the ink residue with a syringe. After rinsing, the cartridge was refilled with ink-dyed water or pure water. Finally, it was sealed again with the refilling hole and the drilled hole glued with a piece of transparent tape.

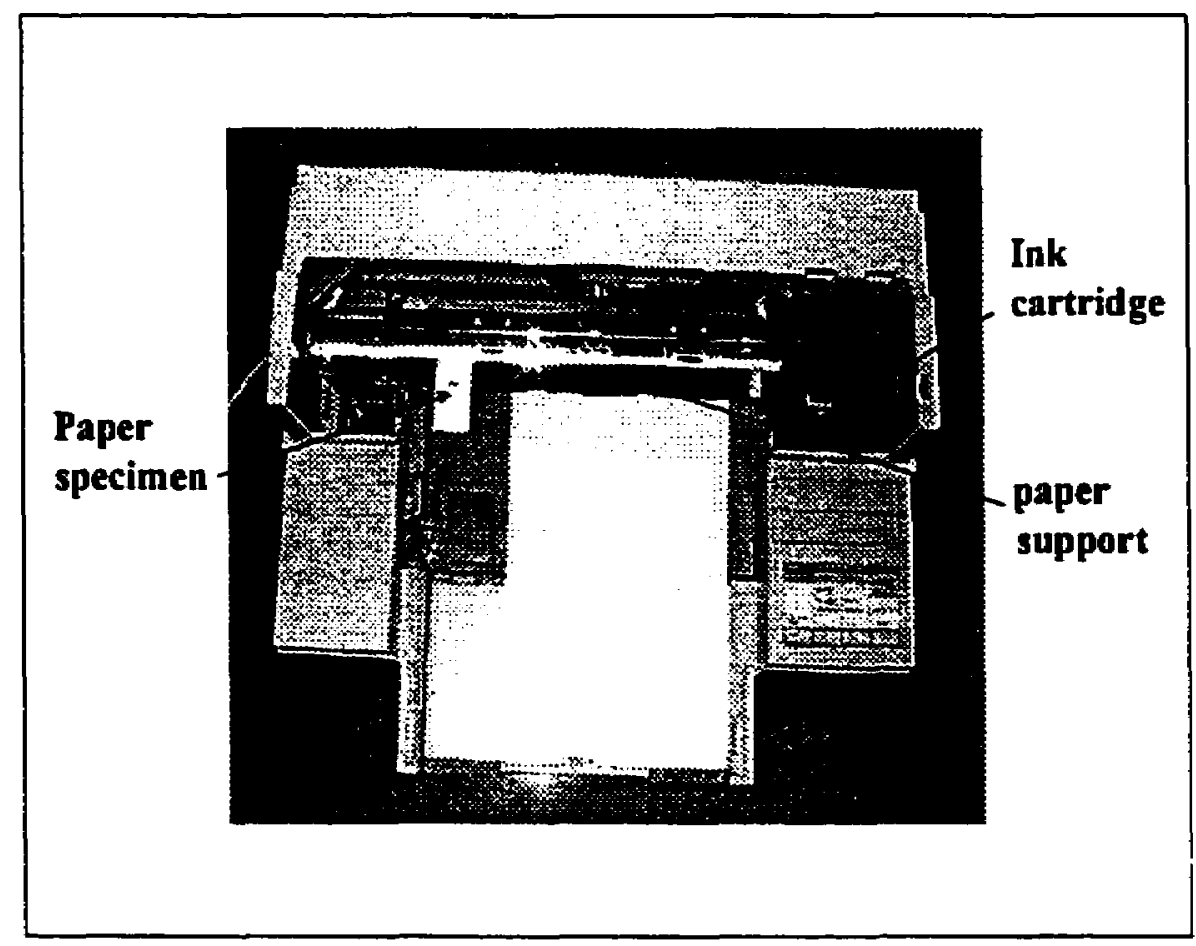

Figure 4.3a. A modified HP 520 inkjet printer 


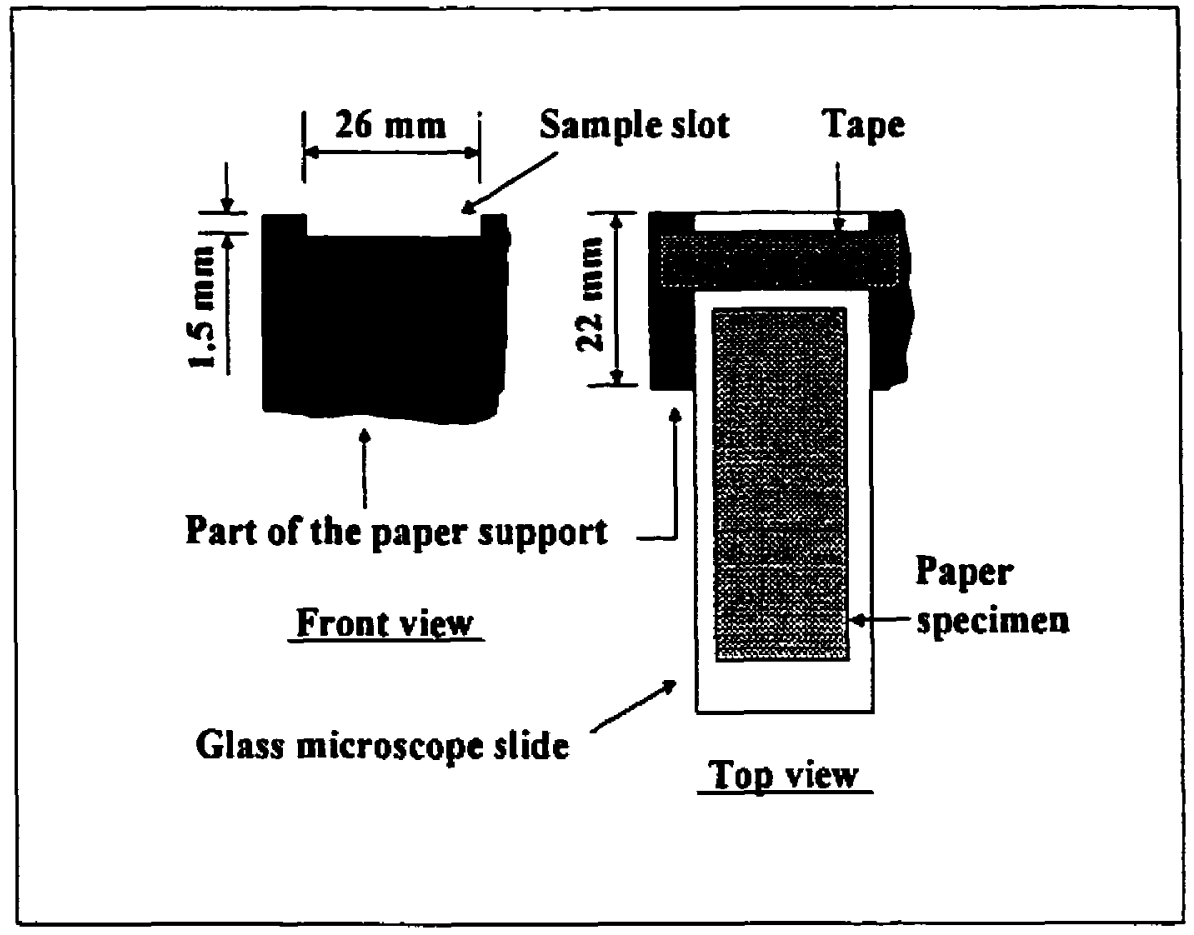

Figure 4.36. Paper specimen with glass microscope slide inserted in the sample slot.

The modified HP 520 inkjet printer is showed in Figure 4.3. The cover of the printer was removed. A slot was machined on the left-top side of the paper support, and a glass microscope slide, on which paper specimen taped, was inserted into the slot and taped in place.

\subsection{DENTIFICATION OF REFERENCE SURFACE}

\subsubsection{PREPARATION OF SPECIMENS}


Within the scanning fieid, for example, in a $603 \times 459 \mu \mathrm{m}$ window, one part of the paper surface was wetted but the surrounding paper remained normally dry. It was assumed that, for the measurement of average step height, the dry area that was not wetted could be used as a stable reference of the wetted area. Before rewetting, there was an average step height between the area to be wetted and the reference surface; after rewetting, there was aiso an average step height between the wetted area and the reference. Therefore, the change in local wetted surface due to rewetting can be judged by comparing the two values of the average step height after and before rewetting. However, it became apparent that the reference area could be affected by the rewetting since the wetting liquid could diffuse far into the surrounding network of cellulosic fibers of the paper.

Because of this, a fixed smooth surface was introduced as a reference for the paper surface. The WYKO Vision $32^{\mathrm{n}}$ software provides the value of average step height between two captured parts in an image, but unfortunately does not provide a sign. It is not possible to assess which part is lower if the surface heights of the two captured areas are close. Therefore, the selected reference surface must be absolutely higher or lower than the paper surface to be examined but not too higher or lower than it since the range of scanning depth of WYKO is $500 \mu \mathrm{m}$. The first option tried was to put a piece of transparent tape $60 \mu \mathrm{m}$ in thickness within the scanning field. But the testing described below indicated that it was not an ideal reference because the wetting liquid diffused into the fibers underneath the tape, litting and debonding 


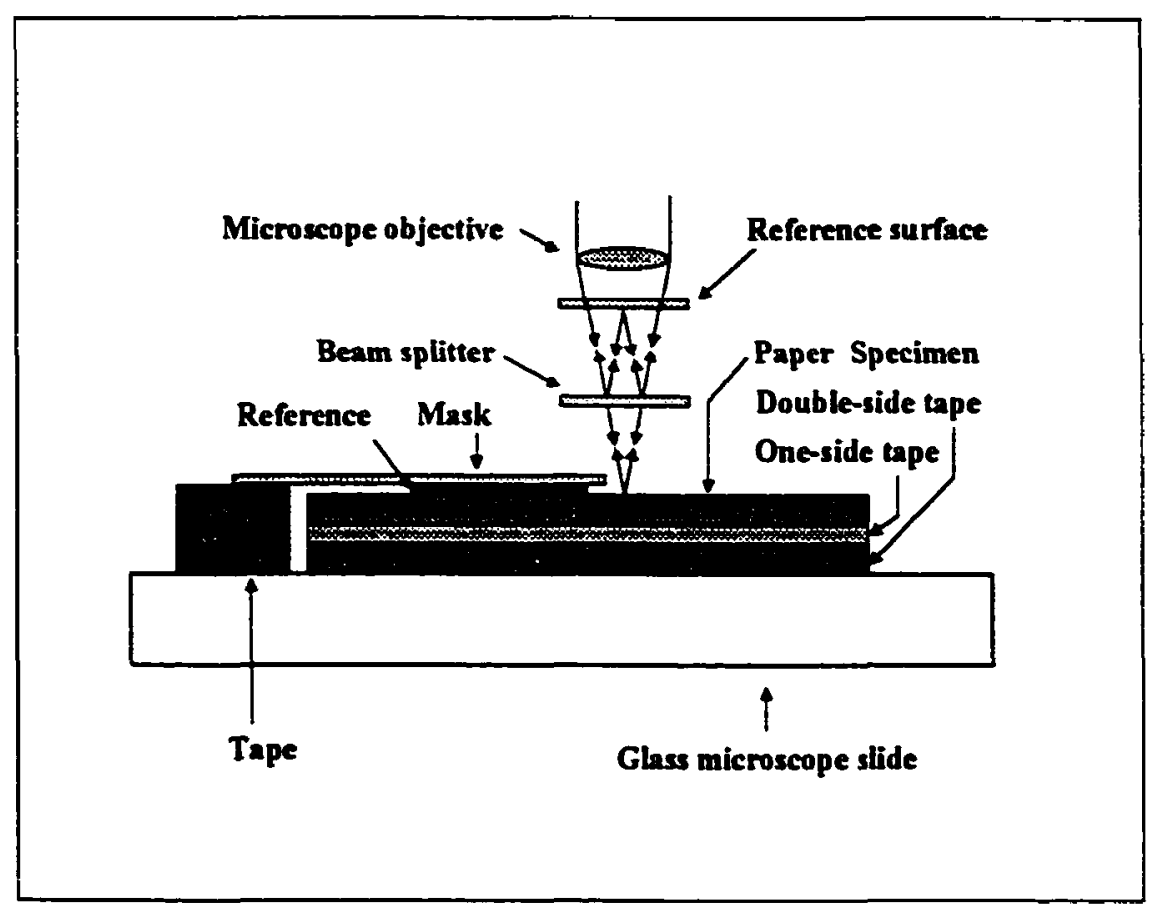

Figure 4.4. Preparation of paper specimen

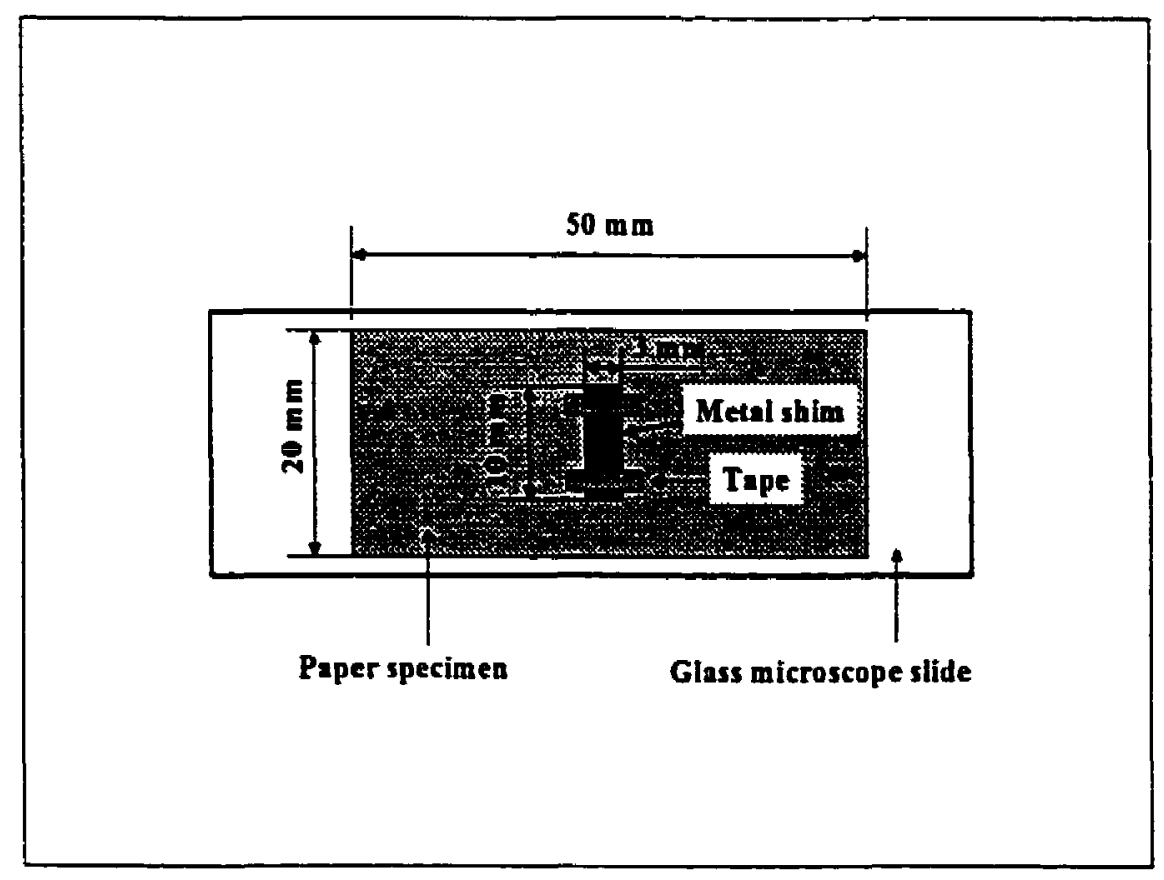

Figure 4.5. Metal shim as reference taped at its two ends onto paper surface 
it. Subsequently, a metal shim was tried since it has a smooth surface and is both rigid and thin. Both transparent tape and metal were evaluated using paper sample TMP No.2 as described below.

The TMP No.2 sample was stored in the constant humidity and temperature room ( $\mathrm{RH}=50 \pm 2 \%, \mathrm{~T}=23 \pm 1{ }^{\circ} \mathrm{C}$ ); and the specimen was also prepared in this room. Paper specimens were cut from sheets. The size of the specimens was $50 \times 20$ $\mathrm{mm}$. The specimens were fixed to glass slides ( $76 \times 25 \mathrm{~mm}$ ) using double-sided tape and single-sided tape to ensure that a flat surface was being studied (see Figure 4.4). A piece of transparent tape was taped directly onto the paper surface as a reference. When the metal shim was used as reference, it was taped at its two edges onto the surface of the paper specimen as Figure 4.5 shows. The size of the reference metal shim was $10 \times 3 \mathrm{~mm}$, and its thickness was $30 \mu \mathrm{m}$.

\subsubsection{TESTING PROCEDURE}

The experiment was carried out in the WYKO laboratory in the Photonics Research Ontario (PRO). The paper specimens were put in the petri dishes with covers and delivered from the constant humidity and temperature room to the WYKO laboratory. The experimental procedure of the testing of the transparent tape and the metal shim as reference surfaces is described as follows: 
a) Opening the valves connecting a nitrogen cylinder and the balance table of the WYKO ${ }^{\text {TM }}$ NT-2000 system. This was important since the balance table would eliminate the vibration.

b) Turning on the WYKO system including the profilometer, the video monitor, and the computer.

c) Leveling the micrometer stage. Putting the specimen on the micrometer stage using the clamp to fix the glass slide on which the specimen was taped.

d) Setting up operation parameters including magnification, scanning depth, and light intensity.

e) Adjusting the position of the specimen using a micrometer, choosing a location with both of the paper surface and the reference within the scanning field.

f) Taping a mask (transparent film) at one end (see Figure 4.4) to cover the reference surface with the edge of the mask extending $100 \mu \mathrm{m}$ beyond the edge of the reference.

g) Pulling off the mask with its one end still taped on the glass slide. Focusing on the scanning field and taking an image of it

h) Taking off the glass slide with the specimen but keeping the micrometer stage and the clamp stationary to ensure the specimen would be relocated at the original position after rewetting. 
i) Inserting and fixing the glass slide with the specimen into the slot of the modified HP 520 inkjet printer. Putting the mask in and using it to cover the reference surface, printing a 2-point line of $5 \mathrm{~mm}$ in length along the width direction of the glass microscope slide onto the scanning field. The wetting liquid in the inkjet cartridge was ink-dyed water with $20 \%$ ink by volume.

j) Taking off the glass slide with the wetted specimen from the printer and putting it back onto the micrometer stage of the WYKO profilometer about 10 minutes later after rewetting, using the clamp to fix it.

k) Taking off the mask. Focusing on the specimen and taking an image of it. Comparing this image to the image taken before rewetting to judge weather it was at the same location. Using the micrometer to adjust the position if there was any change in the location.

1) Using WYKO vision $32^{\text {th }}$ software to analyze both of the two images taken after and before rewetting.

WYKO Vision $32^{\mathrm{TM}}$ software allows one to capture two areas in an image and provides the value of average step height between these two areas. Different pairs of areas can be captured. If the entire reference area is stable, for every pair of captured reference areas and paper surface, the value of the changes in average step height due to rewetting should be the same. The value of average step height between two areas can be obtained before and after rewerting of the paper surface located at the right 
side. To evaluate the suitability of the reference, 5 pairs of captured areas were chosen. The size of each reference area was $720 \times 160 \mu \mathrm{m}$, but the reference was taken at different locations as Figure 4.6. shows. The right area (paper surface) of each pair was at the same location and was also $720 \times 160 \mu \mathrm{m}$. Figure 4.7 is a typical WYKO image for the evaluation testing of the reference surfaces. The size of the scanning field was $1200 \times 890 \mu \mathrm{m}$. The right part in the scanning window was paper surface (TMP No.2); the left part was the reference (transparent tape or metal shim). Figure 4.8 is an example of a pair of the captured reference and paper surface.

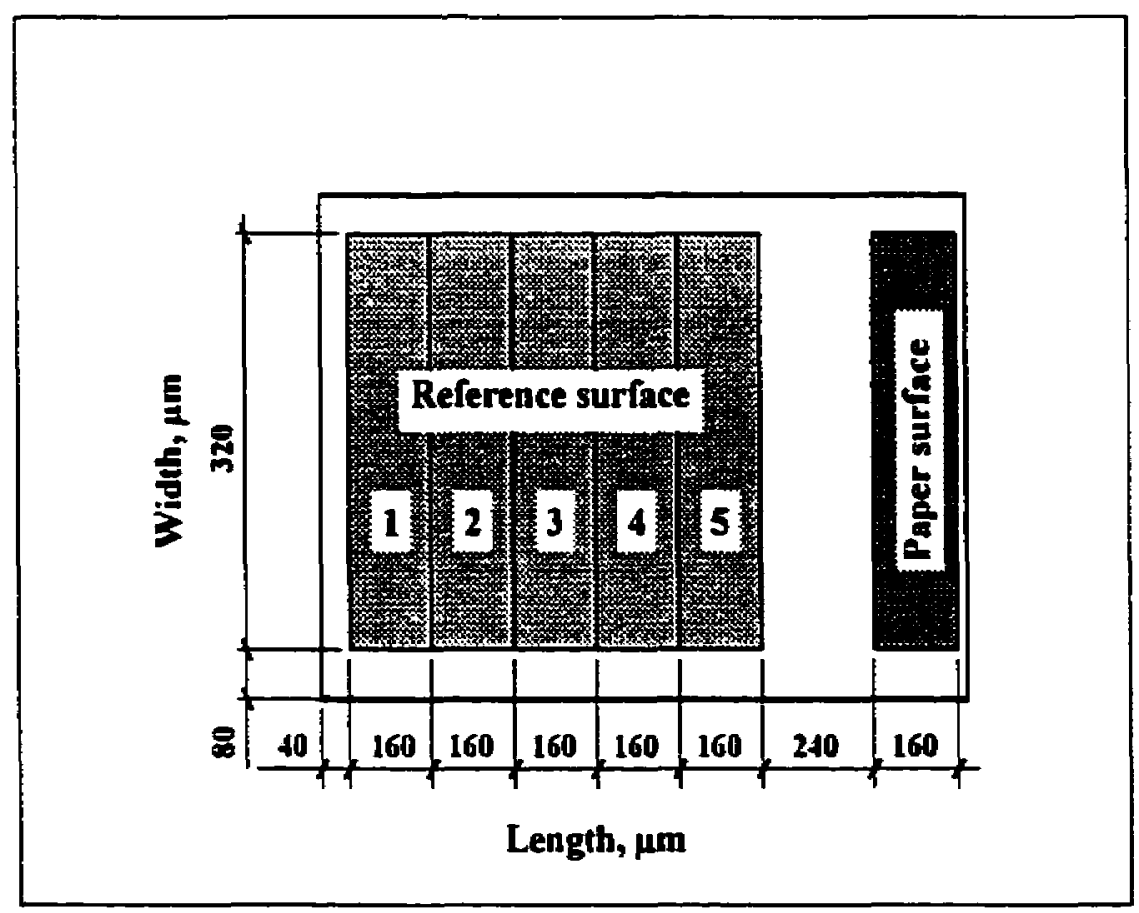

Figure 4.6. The locations of 5 reference areas and the captured paper surface 


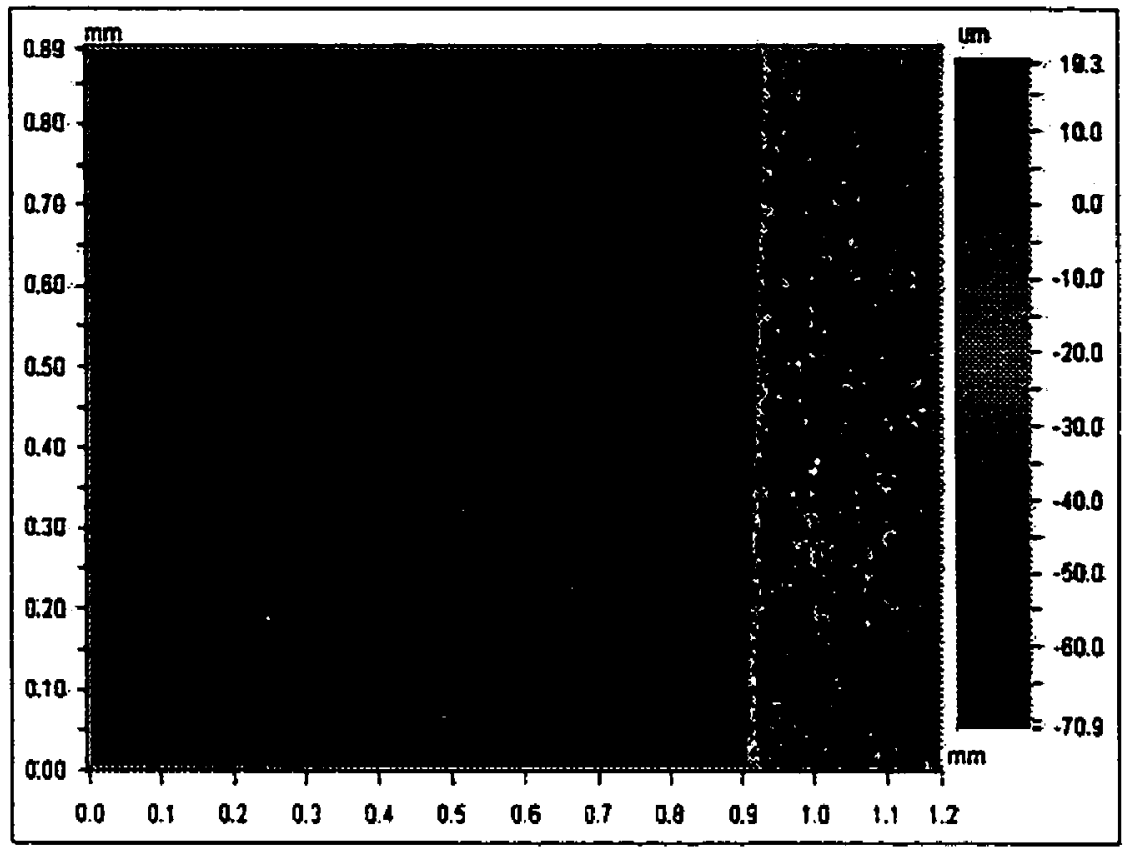

Figure 4.7. Reference testing image. Left is reference, and right is paper surface

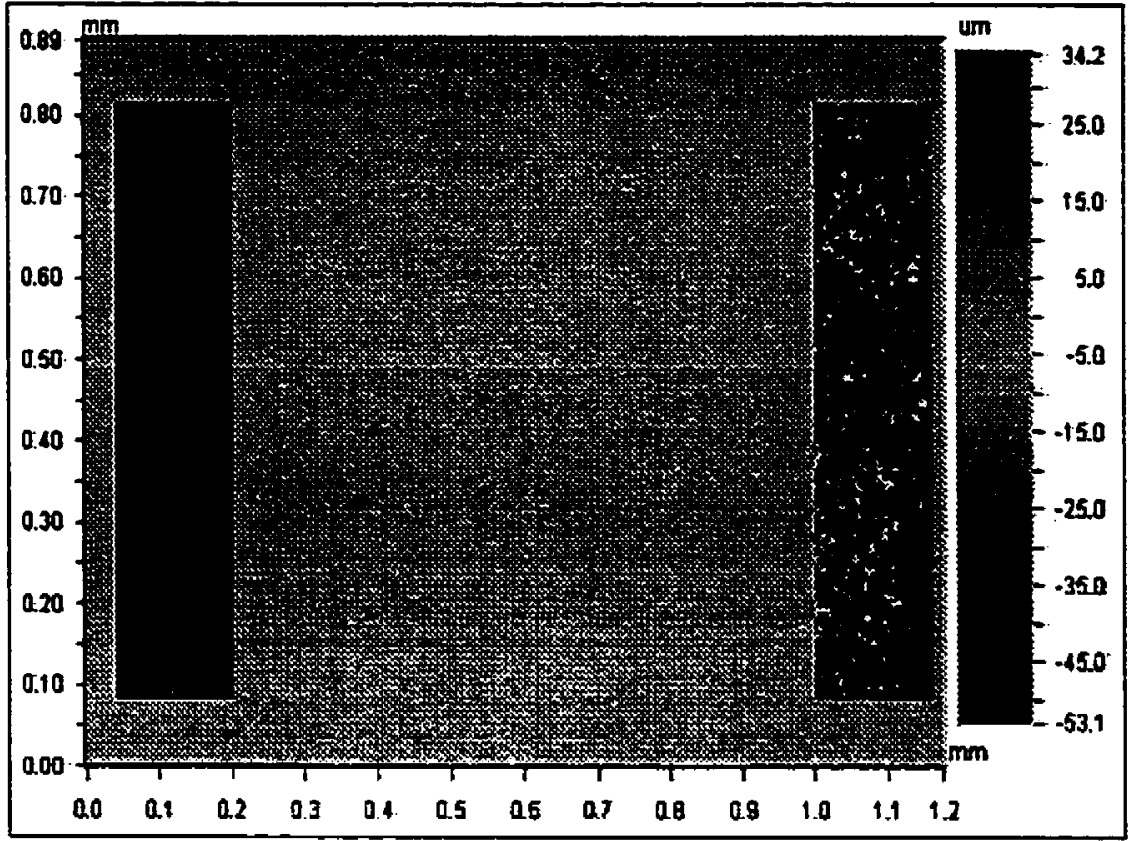

Figure 4.8. Caphured areas of reference and paper surface 


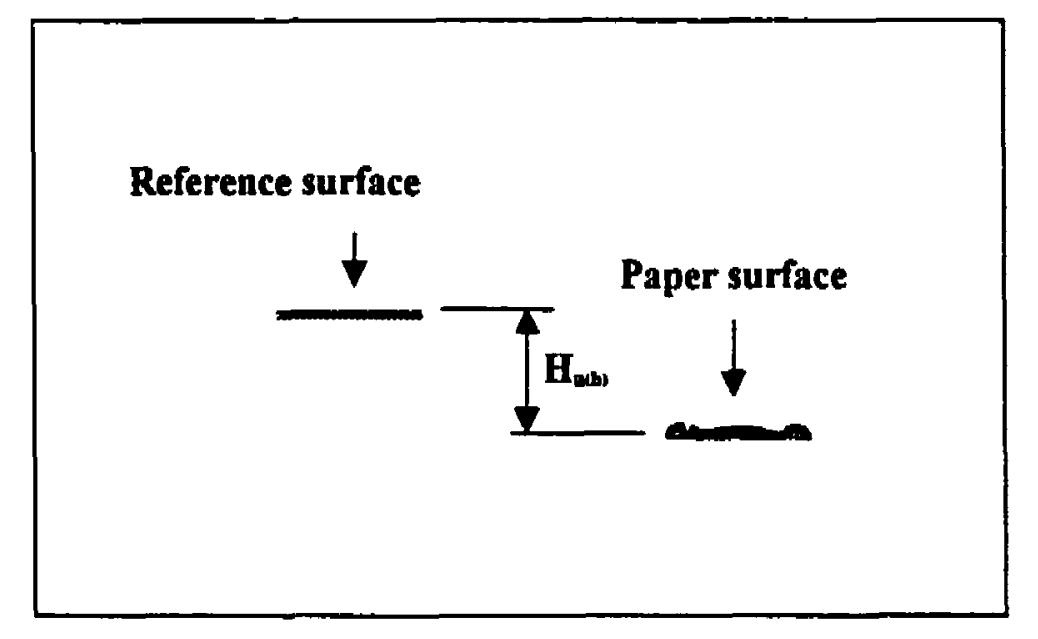

Figure 4.9. Average step height between the reference paper surface

As Figure 4.9 indicates, the surface height of the reference is defined as 0. Therefore, the sign of average step height before wetting is negative since the paper surface is below the reference surface. Thus, the change in average step height $\Delta H$ due to rewetting, i.e., the change in a selected local paper surface height, must be a positive value if the local paper surface is raised by rewetting, as Equation 4.1 describes:

$$
\Delta H=H_{a}-H_{b}
$$

Where $H_{a}$ is the average step height between the reference surface and the paper surface after rewetting, i.e. local paper surface height after rewetting 
(Note that $H_{a}$ was always negative since the reference surface remained above the wetted surface),

$H_{b}$ is the average step height before rewetting, i.e. local surface paper surface height before rewetting.

\subsubsection{EXPERIMENTAL RESULTS}

It was assumed that for every captured reference surfaces, the changes in average step heights, i.e. the changes in local paper surface heights due to rewetting, should be the same if there was no effect of water diffusing on the reference. Table 4.I lists the experimental results of the tests of using transparent tape as a reference. The experiment was repeated 6 times on different specimens. However, the measurement showed that the change after rewetting was not a constant for all captured reference areas. Rather, there was a tendency in all the experimentss, except No.1, that the further the captured reference area was away from the paper surface the smaller the change in average step height. This phenomenon can be explained as follows: Fibers underneath the transparent tape on the left of the scanning field swelled because of the absorption of water diffused from the wetted area on the right; it formed an in-plane internal force in the network of fibers. This force led in an outof-plane force since the dry area on the left stopped the in-plane swelling. 
Table 4.1. The changes in local surface height using different areas (transparent tape) as references

\begin{tabular}{|c|c|c|c|c|c|c|c|}
\hline \multirow{2}{*}{$\begin{array}{c}\text { Expt. } \\
\text { No. }\end{array}$} & \multicolumn{4}{|c|}{$\Delta H, \mu \mathrm{m}$ (Area No. I-5 as reference, respectively) } & Ave. & St.dev. \\
\cline { 2 - 6 } & Ref. 1 & Ref. 2 & Ref.3 & Ref. 4 & Ref. 5 & $\mu \mathrm{m}$ & $\mu \mathrm{m}$ \\
\hline 1 & 4.20 & 4.25 & 4.29 & 4.24 & 4.08 & 4.21 & 0.08 \\
\hline 2 & 2.61 & 2.69 & 3.22 & 3.97 & 4.15 & 3.33 & 0.71 \\
\hline 3 & 3.61 & 4.69 & 6.12 & 8.82 & 9.71 & 6.59 & 2.62 \\
\hline 4 & -12.33 & -7.73 & -3.10 & 1.65 & 3.91 & -3.50 & 6.63 \\
\hline 5 & -7.73 & -4.71 & -1.43 & 2.11 & 3.98 & -1.56 & 4.80 \\
\hline 6 & -6.13 & -3.50 & -1.30 & 1.12 & 2.79 & -1.40 & 3.56 \\
\hline
\end{tabular}

Note: Paper specimen: TMP No.2; wetting liquid: ink-dyed water (20\% ink); wetting method: 2 point line printing; captured reference: $720 \times 160 \mu \mathrm{m}$; captured paper surface: $720 \times 160 \mathrm{~mm}$

The out-of-plane force brought about the rising of the transparent tape, which was glued on the paper surface. For the paper underneath the transparent tape, the further away from the wetted paper area, the bigger the accumulation of the in-plane and outof-plane forces at this position since the fibers were drier and more rigid at the further location from the wetted area. As a result, the rising of the transparent tape at the further location was more. Figure 4.10 explains this possibility. The mechanism behind this phenomenon may be others. Nevertheless, the transparent tape was proved not a good reference surface. 


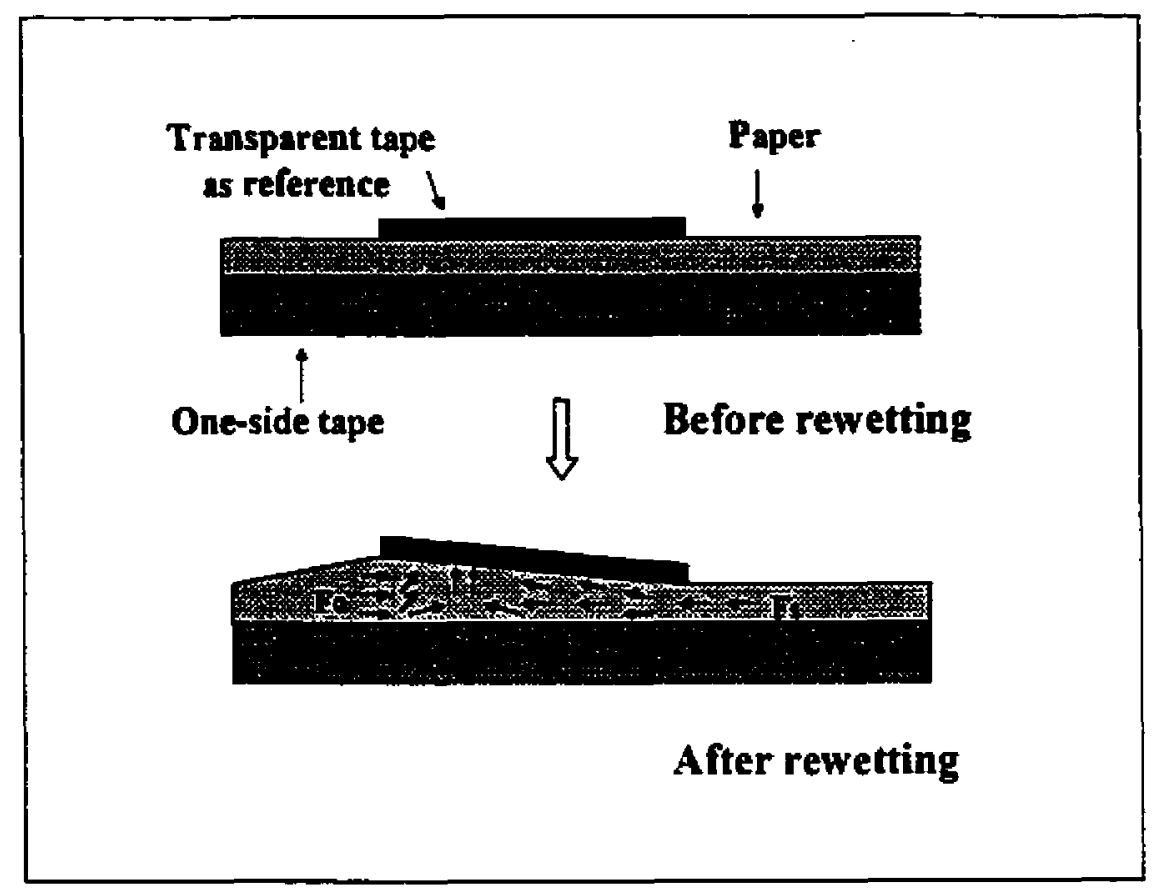

Figure 4.10. Transparent tape raised by intermal forces coused by fiber swelling as a result of water diffusing

After the failure of the transparent tape to sever as reference, a metal shim was tested. The chosen metal shim was a thin steel plate with a thickness of $30 \mu \mathrm{m}$; it was rigid and smooth. A $10 \times 3 \mathrm{~mm}$ piece of this metal shim was taped at its two edges onto the paper surface (see Figure 4.5). Six experiments were conducted on separate specimen. Testing showed the performance of metal shim was excellent (refer to Table 4.2). All the experiments indicated that for different locations of the reference surface the changes in average step height were the same. 
Table 4.2. The changes in local surface height using different areas (metal shim) as references

\begin{tabular}{|c|c|c|c|c|c|c|c|}
\hline \multirow{2}{*}{ Expt. } & \multicolumn{3}{|c|}{$\Delta H, \mu \mathrm{m}$ (Area No.1-5 as reference, respectively) } & Ave. & St.Dev. \\
\cline { 2 - 7 } No. & Ref. 1 & Ref. 2 & Ref.3 & Ref. 4 & Ref. 5 & $\mu \mathrm{m}$ & $\mu \mathrm{m}$ \\
\hline 1 & 4.20 & 4.25 & 4.29 & 4.24 & 4.33 & 4.26 & 0.05 \\
\hline 2 & 7.70 & 7.16 & 6.98 & 6.87 & 6.84 & 3.33 & 0.35 \\
\hline 3 & 2.73 & 2.61 & 2.45 & 2.69 & 2.76 & 2.65 & 0.12 \\
\hline 4 & 2.55 & 2.34 & 2.80 & 3.30 & 3.47 & 2.89 & 0.48 \\
\hline 5 & 9.72 & 9.73 & 9.36 & 9.28 & 9.11 & 9.44 & 0.28 \\
\hline $6\left(5 \mathrm{~min}^{*}\right)$ & 4.72 & 5.11 & 5.06 & 4.63 & 4.83 & 4.87 & 0.21 \\
\hline $6\left(10 \mathrm{~min}^{*}\right)$ & 4.59 & 5.02 & 4.94 & 4.50 & 4.69 & 4.75 & 0.22 \\
\hline $6\left(20 \mathrm{~min}^{*}\right)$ & 4.54 & 5.05 & 4.96 & 4.47 & 4.67 & 4.74 & 0.26 \\
\hline
\end{tabular}

Note: Paper specimen: TMP No.2; wetting liquid: ink-dyed water (20\% ink), wetting method: 2 point line printing; captured reference: $720 \times 160 \mu \mathrm{m}$; captured paper surface: $720 \times 160 \mu \mathrm{m}$; *: the time after rewetting.

The reason for its excellent performance probably lies in that it was not like the transparent tape glued to the paper, but was fixed onto the paper surface with its two ends taped (see Figure 4.5). Because of its rigidity the metal shim arced with the middle part higher than its two ends. Therefore, there was a space between its bottom surface and the paper underneath, which allowed the paper underneath the metal shim to dry immediately once the water of the wetted area of the paper on the right diffused 
into it. As a result, a metal shim fixed onto the paper surface in the way described can be a stable reference surface to the paper surface to be observed.

\subsection{REPEATABILITY TEST}

The investigation of distortion due to local rewetting required WYKO images of the same location on a paper specimen before and after rewetting. However, the paper specimen had to be removed from the WYKO stage for rewetting with the modified inkjet printer, which raised the problem of exact specimen relocation. A micrometer stage with a metal clamp was installed on the WYKO sample stage, to ensure that specimens could be put back to the original location after they were wetted with the printer.

A repeatability test was implemented with TMP No.1 specimen, which was prepared as described in Section 5.4.1, but without a mask. The specimen was fixed with the clamp. During testing, it was repeatedly taken off and put back into the clamp ten times, and the WYKO images were analyzed in terms of arithmetic average roughness $\left(R_{a}\right)$ root mean square roughness $\left(R_{q}\right)$, skewness $\left(R_{s k}\right)$, kurtosis $\left(R_{k w}\right)$, and average step height. The definition of average step height is described in Section 4.3.2 (refer to Figure 4.9). Table 4.3 shows the parameters for the captured $320 \times 320 \mu \mathrm{m}$ 
window on the paper surface. The standard deviations are acceptably small, which means the repeatability of the relocation was good.

Table 4.3. WYKO repeatability test. Sample: TMP No.1. Experiments: 10. Scanning area: $603 X+59 \mu \mathrm{m}$; captured paper area: $320 X 320 \mu \mathrm{m}$.

\begin{tabular}{|l|ccccc|}
\hline & $\mathrm{R}_{\mathrm{a}, \mu \mathrm{m}}$ & $\mathrm{R}_{\mathrm{q}}, \mu \mathrm{m}$ & $\mathrm{R}_{\mathrm{sk}}$ & $\mathrm{R}_{\mathrm{ku}}$ & Ave. St. Ht., $\mu \mathrm{m}$ \\
\hline \multirow{2}{*}{ Average } & 3.219 & 4.575 & 0.766 & 9.511 & -62.801 \\
\multirow{2}{*}{ Standard deviation } & 0.017 & 0.051 & 0.012 & 0.156 & 0.166 \\
\hline
\end{tabular}

\subsection{INVESTIGATION OF LOCAL DISTORTION}

\subsubsection{SAMPLES}

The different grades of paper used as samples in this study are listed in table 4.4 with some of their relevant properties (Table 3.1 includes the full name of these samples and their suppliers). All samples were stored in the constant humidity and temperature room $\left(\mathrm{RH}=50 \pm 2 \%, \mathrm{~T}=23 \pm \mathrm{I}^{\circ} \mathrm{C}\right)$, and the specimens were prepared also in this room. They were put in petri dishes with covers and delivered to the WYKO laboratory when testing needed to be conducted. 
Table 4.4. Samples and some relevant properties.

\begin{tabular}{|l|ccc|}
\hline Sample & Grammage, $\mathrm{g} / \mathrm{m}^{2}$ & Density, $\mathrm{kg} / \mathrm{m}^{3}$ & Roughness PPS, $\mu \mathrm{m}$ \\
\hline TMP No.1 & 35.0 & 350 & 3.80 \\
TMP No.2 & 35.0 & 370 & 3.14 \\
Newsprint & 48.4 & 645 & 3.94 \\
SC & 56.3 & 1208 & 1.09 \\
SS & 75.0 & 759 & N/A \\
IS & 82.0 & 688 & N/A \\
LWC-WO & 60.1 & 1167 & 1.33 \\
LWC-RG & 60.1 & 1219 & 0.69 \\
MWC-WO & 80.8 & 1244 & 0.80 \\
\hline
\end{tabular}

\subsubsection{WETTING METHODS}

A modified HP 520 inkjet printer was used for the rewetting of the paper samples. Two rewetting methods were used: One was a 2-point line, and another was $50 \mu \mathrm{m}$ diameter droplet (a dot of font $\mathrm{l}$ ). Microsoft Word was used for the setup and operation of printing rewetting. A 2-point line of $5 \mathrm{~mm}$ in length or an array of dots of font 1 was drawn at an appropriate location on an A4 size Microsoft Word page. The location of the line or dots corresponded to the position of the paper specimen taped on a glass slide, which was inserted in the slot in the paper support of the modified printer. For 2-point line rewetting, the line was printed along the width $(\mathrm{Y})$ direction 
of the specimen (refer to Figure 4.5), and part of the line was located at the paper surface within the WYKO scanning field. For $50 \mu \mathrm{m}$ diameter droplet rewetting, an array of dots with font 1 was employed since it is not easy to use the modified printer to print a single dot onto the paper surface that is exactly located within the scanning field. The size of the array was $20 \times 3$, which means that there were 20 dots in the width $(\mathrm{Y})$ direction, and 3 dots in length $(\mathrm{X})$ direction. The distances between two dots were about $300 \mu \mathrm{m}$ in both $\mathrm{X}$ and $\mathrm{Y}$ directions, which made it possible to ensure that at least one dot on the paper surface was within the WYKO scanning field.

Because it is impossible to determine the wetted location for dot rewetting with pure water, ink-dyed water was employed. The ink-dyed water was made by mixing distilled pure water together with ink, which was withdrawn from the HP $51625 \mathrm{~A}$ cartridge. First, ink-dyed water with ink of $10 \%$ by volume was tried, but the printed dots were not black enough to be identified optically. Therefore, ink-dyed water with $20 \%$ ink by volume was used, and the dots could be identified because of their slightly black color.

The amount of ink-dyed water applied to paper was measured by weighing the cartridge before and after printing. Since the volume of ink-dyed water used for printing a line or a dot is very small, 4 areas with size of $138 \times 35 \mathrm{~mm}$ and $10^{6}$ dots of font 1 were printed. The average values of ink-dyed water per dot were obtained by comparing the cartridge weights before and after printing. For 2-point line 
rewetting, the amount of ink-dyed water applied to paper was $10.22 \mathrm{~g} / \mathrm{m}^{2}$, and for font 1 dot rewetting, it was $0.0416 \mu \mathrm{g} / \mathrm{dot}$; therefore, the diameter of a spherical ink-dye water droplet coming from the cartridge to produce a dot of font 1 was about $50 \mu \mathrm{m}$.

Pure water was also used for the evaluation of the effect of pigment particles, which may exist in the ink, on the wetting liquid and the distortions caused by the wetting liquid. The wetting method was 2 point line printing rewetting. Testing showed that there was no significant difference between ink-dyed water and pure water when they are used as wetting liquids. This was expected since the content of the ink in the ink-dyed water was low. The experimental data is presented in Chapter Six.

\subsubsection{EXPERIMENTAL PROCEDURES}

The distortions caused by local rewetting were investigated in terms of the changes of average arithmetical roughness $(\mathrm{Ra})$, root mean square roughness $(\mathrm{Rq})$, skewness $\left(\mathbf{R}_{\mathbf{s k}}\right)$, kurtosis $\left(\mathbf{R}_{\mathbf{k u}}\right.$ ), and average step height. The preparation of the specimens is described in Section 4.3.1, and the metal shim was used as the reference. The experimental procedure was similar to the procedure employed for the testing of references (Section 4.3.2). The differences were: 
a) The total scanning field was $603 \times 459 \mu \mathrm{m}$. This means higher magnification and higher in-plane resolution (i.e., 2 times) were used than for reference testing.

b) The size of the metal reference surface was about one-third of the scanning area.

c) For 2-point line printing, the captured the area of the paper surface was $320 \times 320 \mu \mathrm{m}$ while the captured area of the reference surface was $360 \times$ $100 \mu \mathrm{m}$. For $50 \mu \mathrm{m}$ droplet rewetting, the captured areas of both paper and reference were $80 \times 80 \mu \mathrm{m}$.

d) For $50 \mu \mathrm{m}$ droplet rewetting, an optical fiber light was used to identify the wetted areas, which were slightly darker since the wetting liquid was inkdyed water.

The images after rewetting were taken 10 minutes later after the specimens were rewetted with the printer, which ensured that the specimens were completely dried again. The delay of 10 minutes was based on the experimental testing results using the metal shim as reference. Experiment No. 6, presented in Table 4.2, shows that the changes in average step height due to rewetting are constant 10 minutes after rewetting.

Figure 4.11 is a typical WYKO image for the investigation of distortions caused by 2-point line rewetting. Figure 4.12 is the image with two selected areas; the 


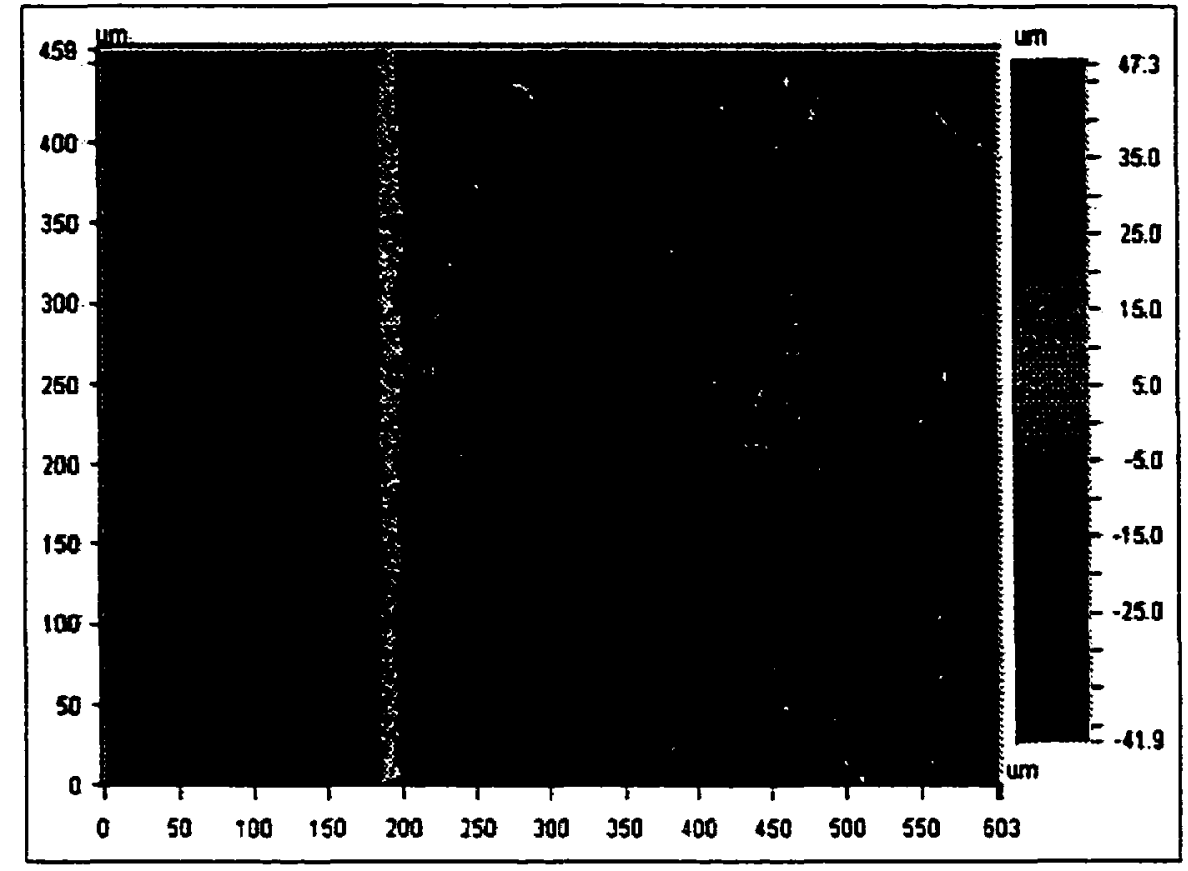

Figure 4.11. WYKO image. The left is reference; the right is paper surface

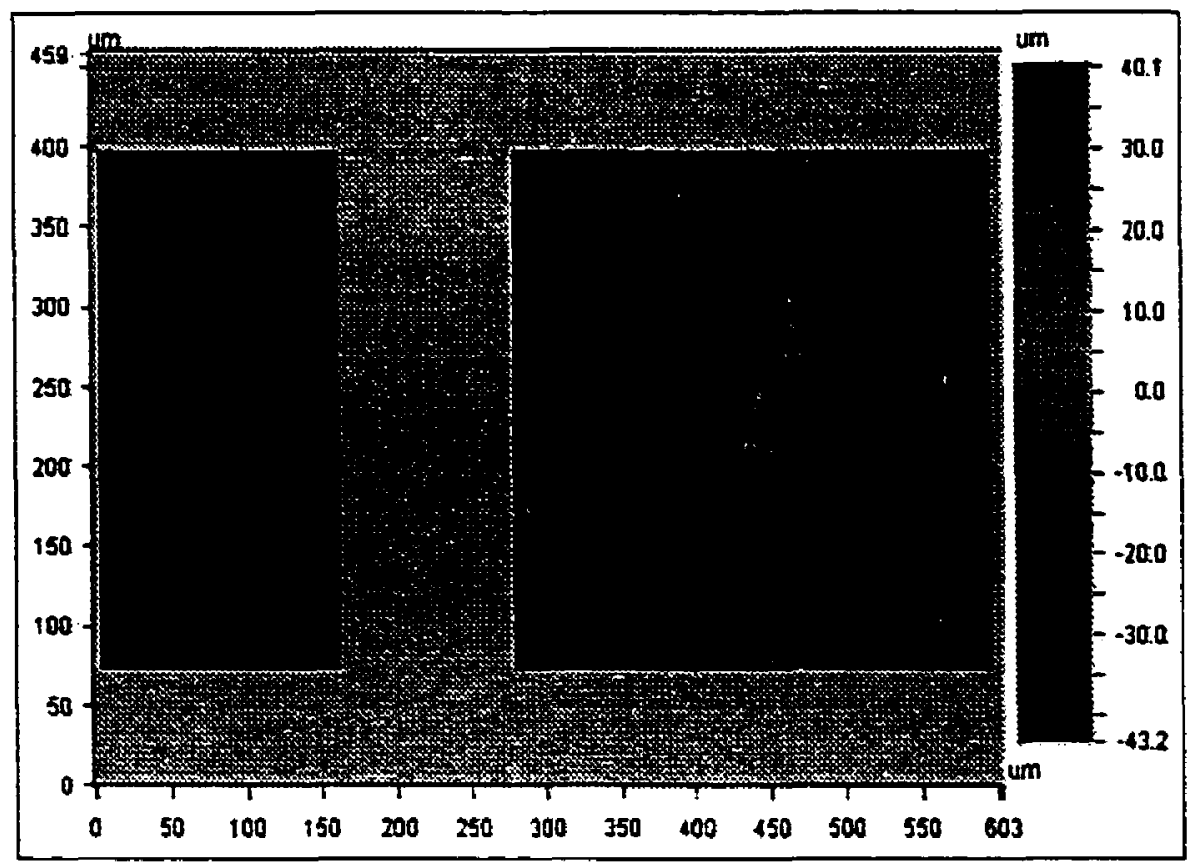

Figure 4.12. WYKO image of two captured areas 
right part is a window of the paper surface to be examined, and the left part is the metal surface as reference. 


\subsection{INTRODUCTION}

Fiber-to-fiber bonds play an important role in the behavior of the paper surface during the application of aqueous liquids in coating and printing. It was hypothesized that fiber-to-fiber bond strength is one of the important characteristics that affects the distortion of paper when it contacts water. In order to investigate the relationship between the changes in local surface height due to local rewetting and relevant paper properties, the fiber-to-fiber bond strength for various grades of uncoated paper was measured in this study. A method developed by Skowronski and Bichard [53] was used for the measurement.

\subsection{PREPARATION OF SPECIMENS}

Six uncoated samples including TMP No.1, TMP No.2, surface sized copy paper, internally sized copy paper, newsprint, and super calendered paper were tested in this study. Specimens were prepared by applying clear, colorless adhesive tape (transparent packaging tape, $50 \mathrm{~mm}$ wide, Manco Inc.) to both sides of a strip of 
paper. As Figure 5.1 shows, the dimension of the specimens was $120 \mathrm{~mm}$ long and initially $30 \mathrm{~mm}$ wide, trimmed to $25 \mathrm{~mm}$ wide. The length of the paper specimen was parallel to machine direction. On both sides of the specimen, the excess adhesive tape was folded back on itself at one end to provide a position to grip the specimen. In order to ensure uniform contact of the adhesive tape to the sample, the prepared specimen was pressed between two smooth glass plates for 10 minutes with a $1.5 \mathrm{~kg}$ weight on the upper glass plate.

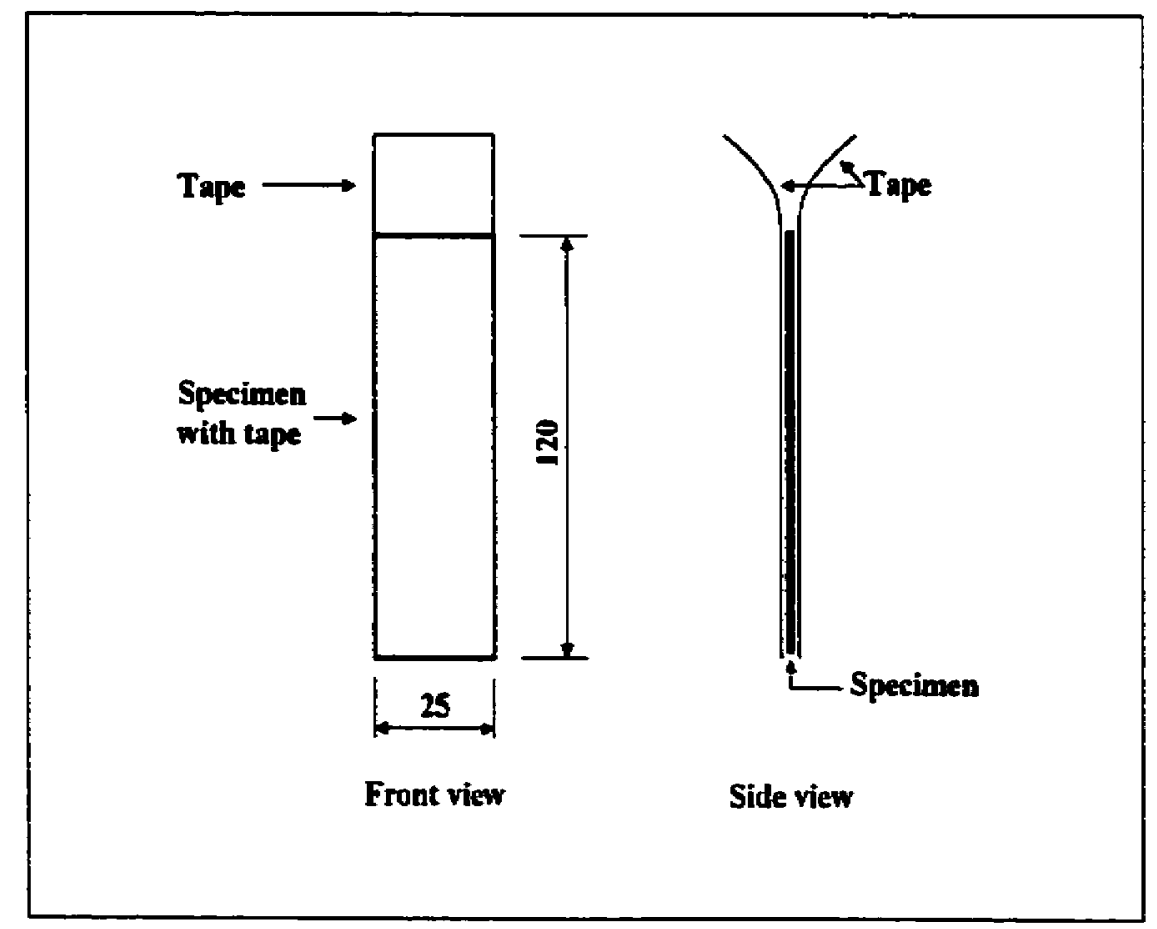

Figure 5.1. Preparation of specimen for the measurement of bond strength 


\subsection{EXPERIMENTAL SETUP}

The experimental setup is shown in Figure 5.2. The fiber-to-fiber bond strength experiments were performed on a Sintech testing machine with a $50 \mathrm{lb}$ load cell and a free-rotating wheel assembly. The wheel was $100 \mathrm{~mm}$ in diameter and $25 \mathrm{~mm}$ wide. The specimen was attached onto the wheel using masking tape and fastened with the upper transparent tape grip clamped in the upper jaws connected to the load cell. The wheel attachment was clamped to the lower jaws of the Sintech. The tests were conducted in the constant humidity and temperature room $(\mathrm{RH}=50 \pm$ $2 \%, T=23 \pm\left({ }^{\circ} \mathrm{C}\right)$.

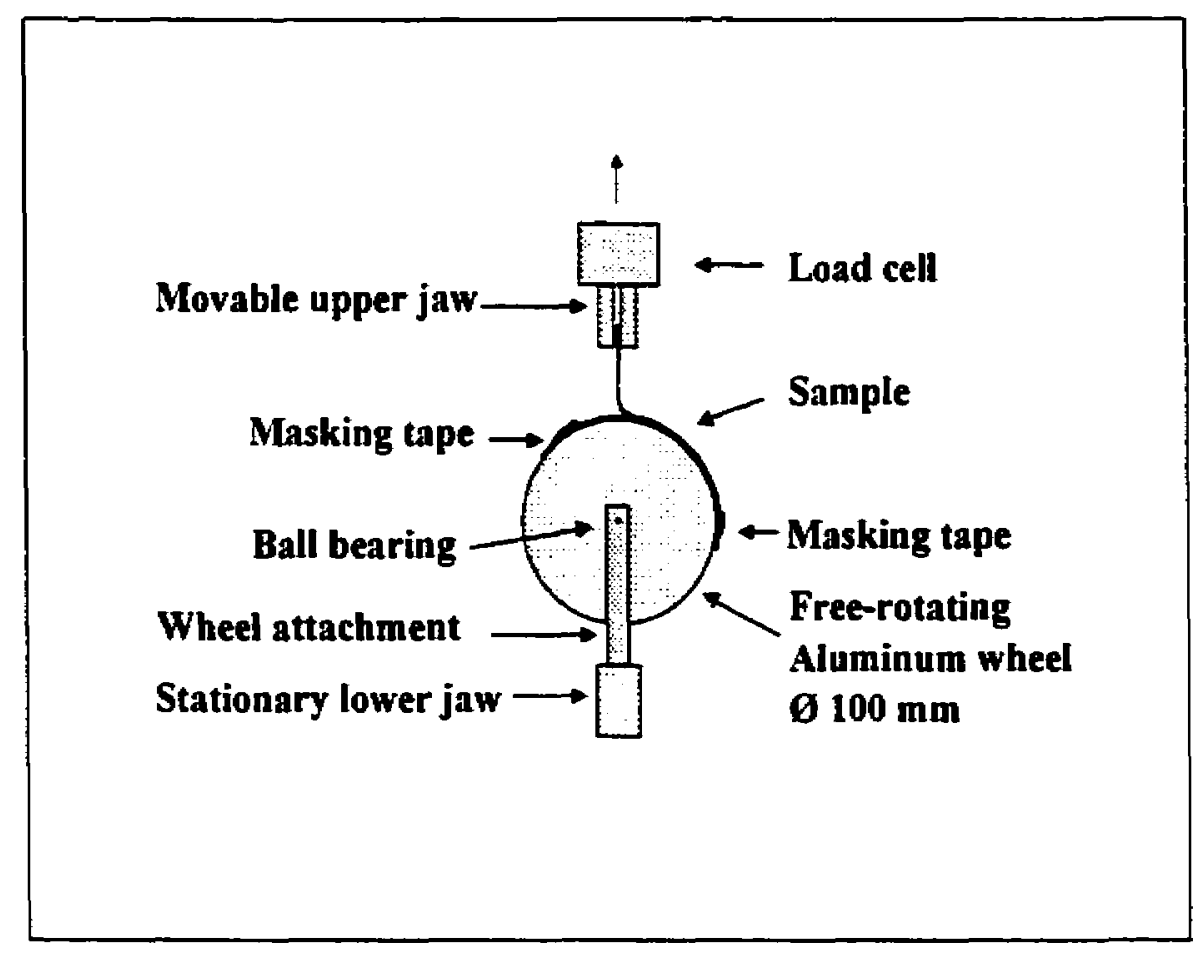

Figure 5.2. Bond strength testing assembly. 


\subsection{MEASUREMENT OF BOND-BREAKING ENERGY}

During the test, the specimen was delaminated by pulling the upper transparent tape at a constant rate of $20 \mathrm{~mm} / \mathrm{min}$. The force required to delaminate the

specimen (i.e bond-breaking force) was data logged and recorded as a function of delaminated specimen length as shown in Figure 5.3. The bond-breaking energy was determined from the area under the curve between $40 \mathrm{~mm}$ and $80 \mathrm{~mm}$ of delaminated length. For each sample, six measurements were conducted.

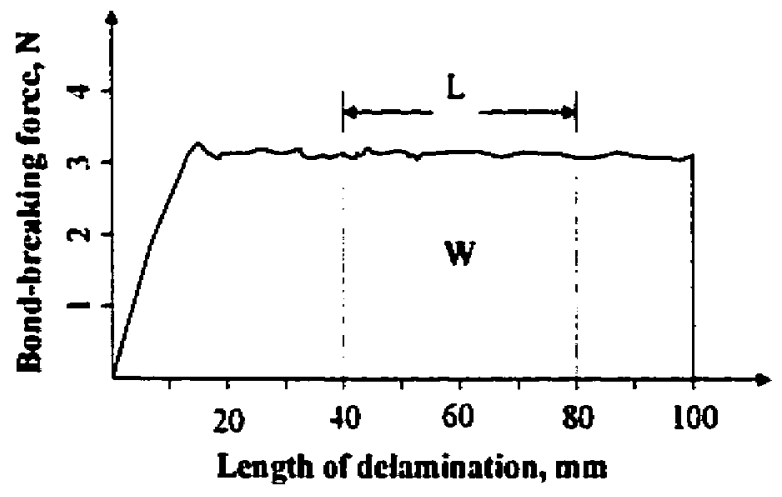

Figure 5.3. Graph of the force required for specimen delamination 


\subsection{BOND STRENGTH}

Bond strength is defined as the breaking energy per unit area of the delamination, and it is calculated from the following equation:

$$
W_{b}=W /(L b)
$$

where $W_{b}$ : bond strength, $\mathrm{J} / \mathrm{m}^{2}$

$W$ : bond-breaking energy, $\mathrm{J}$

$L$ : Length of delamination, $0.04 \mathrm{~m}$

b: Specimen width, $0.025 \mathrm{~m}$.

Table 5.1. Fiber-10-fiber bond strength of uncoated paper ( six measurements for each sample)

\begin{tabular}{|l|c|cccccc|}
\hline \multicolumn{2}{|c|}{ Sample } & SS & IS & Newsprint & TMP No.1 & TMP No.2 & SC \\
\hline$W_{\mathrm{b}}$ & Average & 71.85 & 54.73 & 49.25 & 48.95 & 48.64 & 44.98 \\
\cline { 2 - 8 } $\mathrm{J} / \mathrm{m}^{2}$ & Std. Dev. & 2.56 & 1.94 & 0.99 & 1.23 & 1.39 & 1.17 \\
\hline
\end{tabular}

Table 5.1 presents the experimental results. SS stands for surface sized copy paper, and IS for internally sized copy paper (see Table 3.2). As we can see in Table 5.1, the samples made of mechanical pulp (newsprint, TMP No.1, and TMP No. 2) had similar bond strengths. The two sized copy papers had relatively greater bond 
strength, especially the surface sized copy paper. The reason might be that the sizing agents such as starch and rosin, which have an affinity for fibers, were sorbed onto fibers, strengthening fiber-to-fiber and fiber-filler bonds. The high pressure of supercalendering resulted in the densification of the fibers themselves, leading to the reduction in space between fibers [27], and therefore the supercalendering paper (SC) showed the smallest value of bond strength among all the samples. 


\section{CHAPTER SIX}

\section{RESULTS AND DISCUSSION}

\subsection{INTRODUCTION}

The method described in Chapter Four was used to investigate local distortions caused by local rewetting. Local distortions were quantified in terms of the changes in arithmetic average roughness, root mean roughness, skewness, kurtosis, and average step height. In order to understand the intrinsic factors that affect the local distortions, the relationships between local distortions and the paper properties were analyzed. Local surface roughness, fiber-to-fiber bond strength, and sheet density were considered to be the main parameters that play important roles in the local distortions due to local rewetting.

\subsection{EFFECT OF WATER}

\subsubsection{CHANGES IN ROUGHNESS}

The changes in arithmetic average roughness ( $\mathrm{Ra}$ ) and root means square roughness $(\mathrm{Rq})$ due to rewetting are presented in Figure 6.1 and 6.2. Figure 6.1 is the results of 2-point line rewetting, and Figure 6.2 is for $50 \mu \mathrm{m}$ droplet rewetting. 


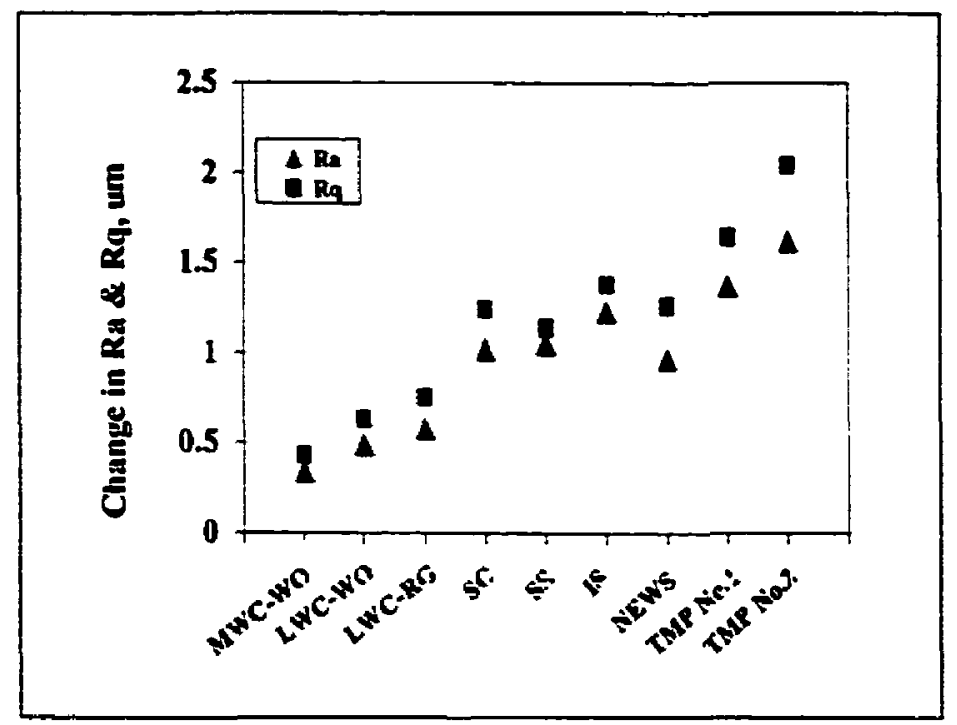

Figure 6.1. Changes in roughness vs. types of paper. (2-point line rewetting; wetting liquid: ink-dyed water (20\% ink); captured area: $320 \times 320 \mathrm{jm}$ ).

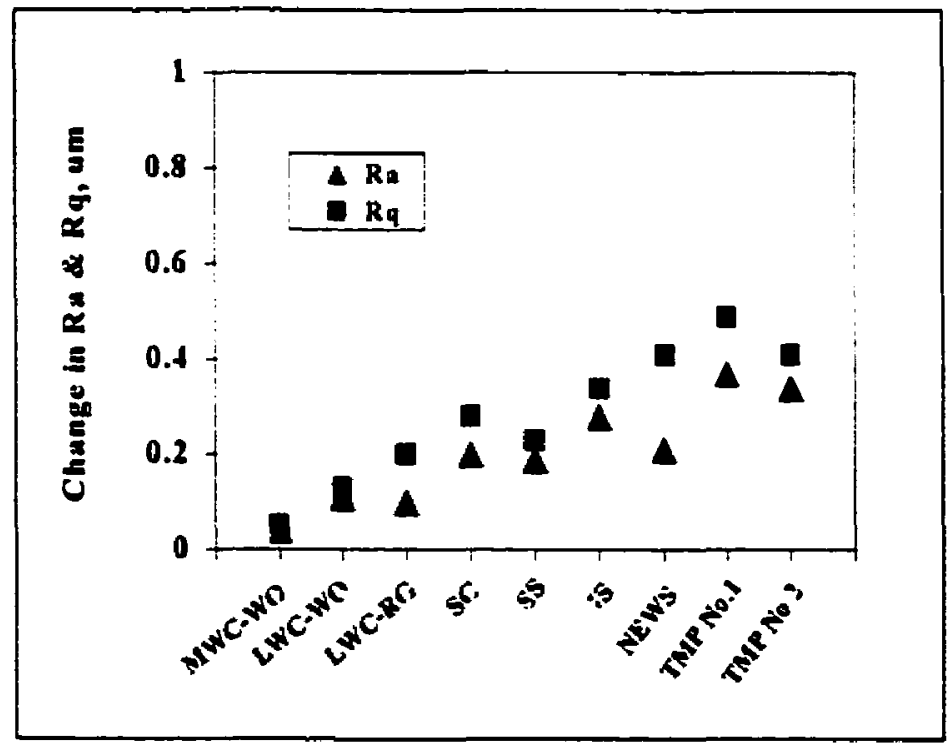

Figure 6.2. Changes in roughness vs. types of paper. $(50 \mathrm{~cm}$ droplet rewetting, wetting liquid: ink-dyed water (20\% ink); captured area: $80 \times 80 \mu \mathrm{m})$. 
It is evident that the increase in the roughness of coated paper was less than that of uncoated paper, which suggests that coating prevents water from penetrating into the substrate. The greater the coating weight, the lesser the expected sheet roughening, and the MWC-WO sample had the least change in roughness. Fibers of the uncoated paper absorbed more water during rewetting compared to the coated paper, leading to more roughening. However, since sizing reduces the paper porosity, and it reinforce the resistance of paper to water, both surface sized and internally sized papers showed smaller changes in roughness.

The definitions of arithmetic average roughness $\left(R_{a}\right)$ and root mean square $\left(R_{q}\right)$ are given in Section 2.1.3. If a surface has a profile that contains no large deviations from the mean surface or line, the values of $R_{a}$ and $R_{q}$ will be similar. However, if there are appreciable numbers of large bumps or holes, the largest values of the profile function will dominate the surface statistics and $R_{q}$ will be larger than $\mathbf{R}_{\mathrm{a}}$. The results presented in Figure 6.1 and 6.2 indicate that $\mathbf{R}_{\mathbf{q}}$ increased more than $\mathbf{R}_{\mathbf{a}}$ for all samples. Generally speaking, the difference between the increases in $R_{q}$ and $R_{a}$ of uncoated papers are larger than that of coated papers. As well, among the uncoated samples, the sized papers showed relatively smaller difference between the increases in $R_{q}$ and $R_{a}$. This means that the surface structure of uncoated and unsized 
papers became more irregular with more bumps or valleys, but coating and sizing reduced this tendency.

Table 6.1. T-test results of changes in roughness due to rewetting on different grades of paper

\begin{tabular}{|l|cc|cc|}
\hline & \multicolumn{2}{|c|}{2 point line rewetting } & \multicolumn{2}{c|}{$50 \mu \mathrm{m}$ D droplet rewetting } \\
\cline { 2 - 5 } & Change in Ra & Change in Rq & Change in Ra & Change in Rq \\
\hline MWC-WO & $* * *$ & $* * *$ & $*$ & $* *$ \\
LWC-WO & $* * *$ & $* * *$ & $* * *$ & $* * *$ \\
LWC-RG & $* * *$ & $* * *$ & 1 & 1 \\
NEWS & $* * *$ & $* * *$ & $* *$ & $* *$ \\
SC & $* * *$ & $* * *$ & $* *$ & $* *$ \\
SS & $* * *$ & $* * *$ & $* *$ & $* *$ \\
IS & $* * *$ & $* * *$ & $* *$ & $* *$ \\
TMP No.1 & $* * *$ & $* * *$ & $* * *$ & $* * *$ \\
TMP No.2 & $* * *$ & $* * *$ & $* * *$ & $* * *$ \\
\hline
\end{tabular}

Note: /: The change is not significant at $90 \%$ confidence level

$\because$ The change is significant at $90 \%$ confidence level

**: The change is significant at $95 \%$ confidence level

***: The change is significant at $99 \%$ confidence level

As expected, 2-point line rewetting brought about larger increase in roughness than $50 \mu \mathrm{m}$ droplet rewetting even though roughness was measured in an area which was completely wet in both cases. The reason may be that the wetted area for $50 \mu \mathrm{m}$ 
droplet rewetting was relatively small, and the surrounding dry area restrains the changes in structure within the wetted field. In addition, the amount of water applied and hence the wetting depth for the $50 \mu \mathrm{m}$ droplet rewetting was much less than that for the 2-point line rewetting (refer to Section 6.2.4).

Table 6.1 is the t-test results of the changes in roughness for both rewetting methods. For 2-point line rewetting, the increases in roughness including $\mathbf{R}_{\mathbf{a}}$ and $\mathbf{R}_{\mathbf{q}}$ were significant at $95 \%$ confidence level. For $50 \mu \mathrm{m}$ droplet rewetting, even though the changes were relatively small, there were also significant increases in roughness except the increases in $R_{a}$ of $M W C-W O$ and in $R_{a}$ as well as $R_{q}$ of LWC-RG.

\subsubsection{CHANGES IN SKEWNESS AND KURTOSIS}

Figure 6.3 shows the initial skewness $\left(\mathbf{R}_{\mathrm{sk}}\right)$ of a window of the paper surface before rewetting. As we can see, the skewness of the coated samples as well as supercalendered paper (SC) were negative while most of the uncoated samples had a positive skewness. This means that the coated papers had fewer bumps or peaks above the mean surface, and the supercalendered paper had relatively more valleys. Uncoated, uncalendered papers had larger $\mathbf{R}_{\text {sk }}$ indicating a "peaky" surface. After rewetting including both 2-point line and $50 \mu \mathrm{m}$ droplet methods, the skewness of all 


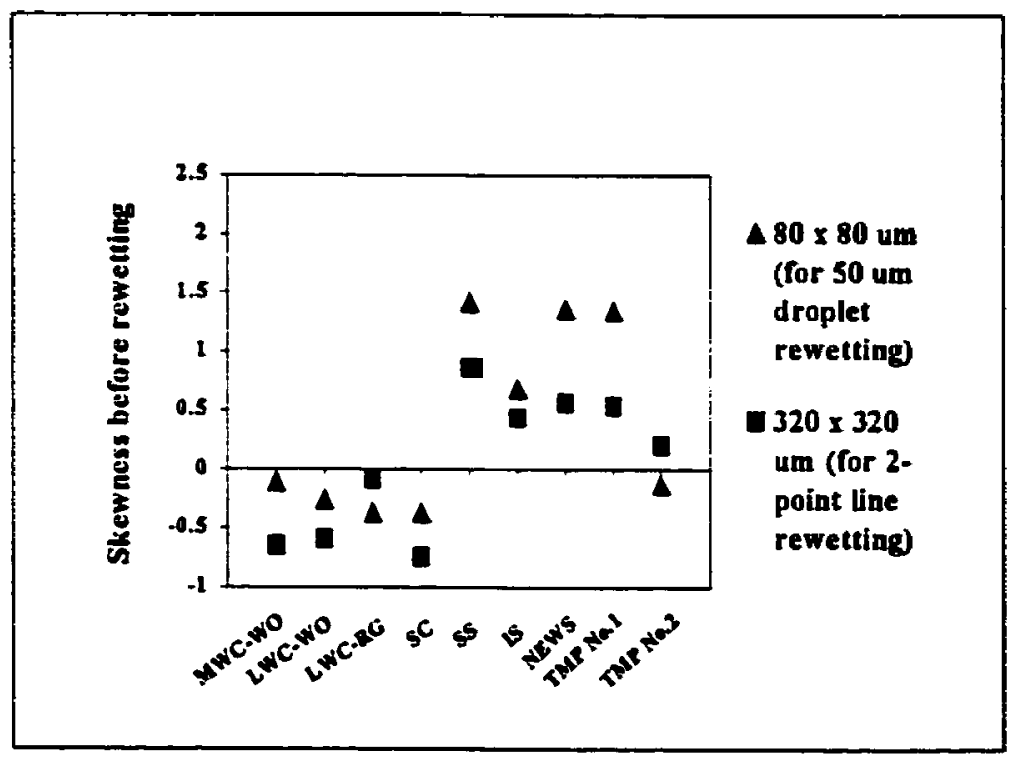

Figure 6.3. Skewness before rewetting vs. types of paper. (For 2-point line rewetting, the captured area was $320 \times 320 \mu \mathrm{m}$; for $50 \mu \mathrm{m}$ droplet rewetting, the captured area was $80 \times 80 \mu \mathrm{m})$.

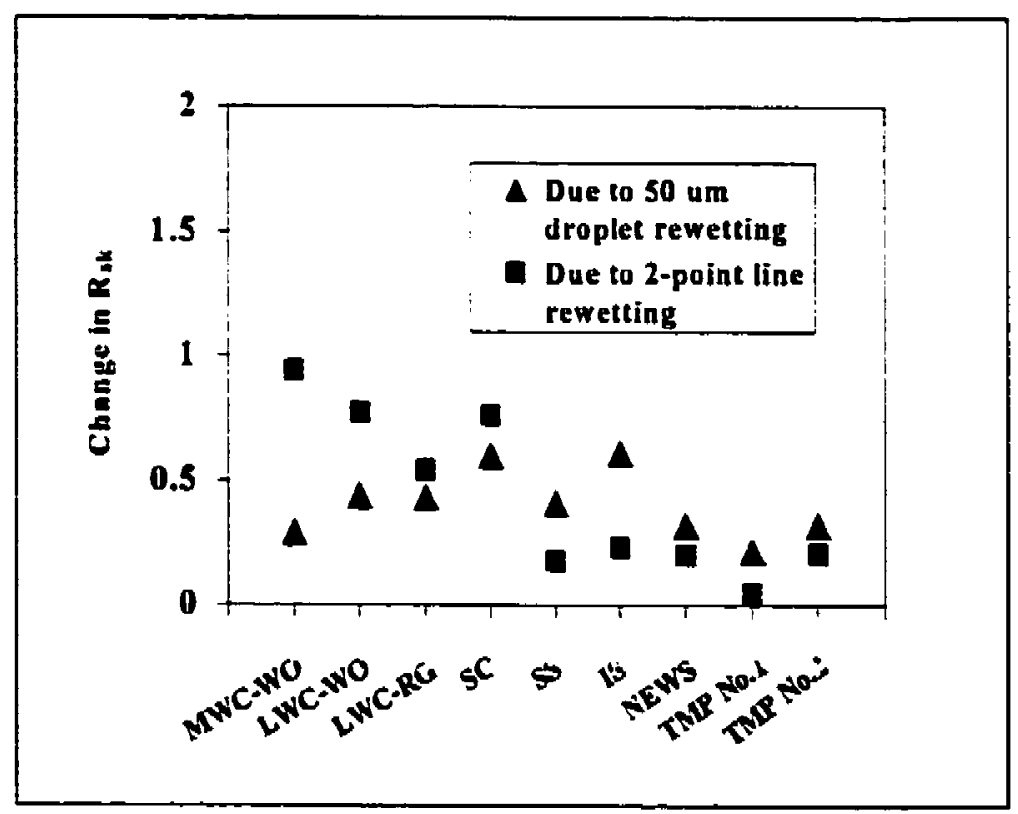

Figure 6.t. Change in skewness due to rewetting vs. types of paper. (For 2-point line rewetting, the captured area was $320 \times 320$ pm: for $50 \mu \mathrm{m}$ droplet rewetting, the captured area was $80 \times 80 \mu \mathrm{m}$ ). 
the samples increased as Figure 6.4 indicates. The increase of skewness in coated papers may due to the swelling of the fibers underneath the coating layer, leading to more bumps. For supercalendered paper, the collapsed fibers tended to recover their natural shape when wet [6], leading to an increase in the number of peaks and hence an increase in $\mathbf{R}_{\text {sk. }}$.

Kurtosis $\left(\mathbf{R}_{\mathbf{k u}}\right)$ is a measure of the randomness of profile heights (refer to Section 2.1.3). A perfectly Gaussian or random surface has a kurtosis of 3 , and a kurtosis value greater than 3 represents that a surface has an appreciable number of high and low extremes. Figure 6.5 shows the initial kurtosis of a window of the paper surface to be wetted. Basically, the kurtosis values of the coated papers were closer to 3 than those of the uncoated samples, which means the surface heights of the coated papers were more randomly distributed compared to the uncoated samples. After rewetting with both methods, as Figure 6.6 shows, the kurtosis decreased in all the samples except LWC-RG. This suggests that rewetting resulted in fiber swelling and consequently rising, leading to more peaks, but that they were relatively more randomly distributed in different surface heights compared to before rewetting, leading to a decrease in $\mathbf{R}_{\mathbf{k u}}$. The uncoated papers decreased more in kurtosis than the coated papers. 


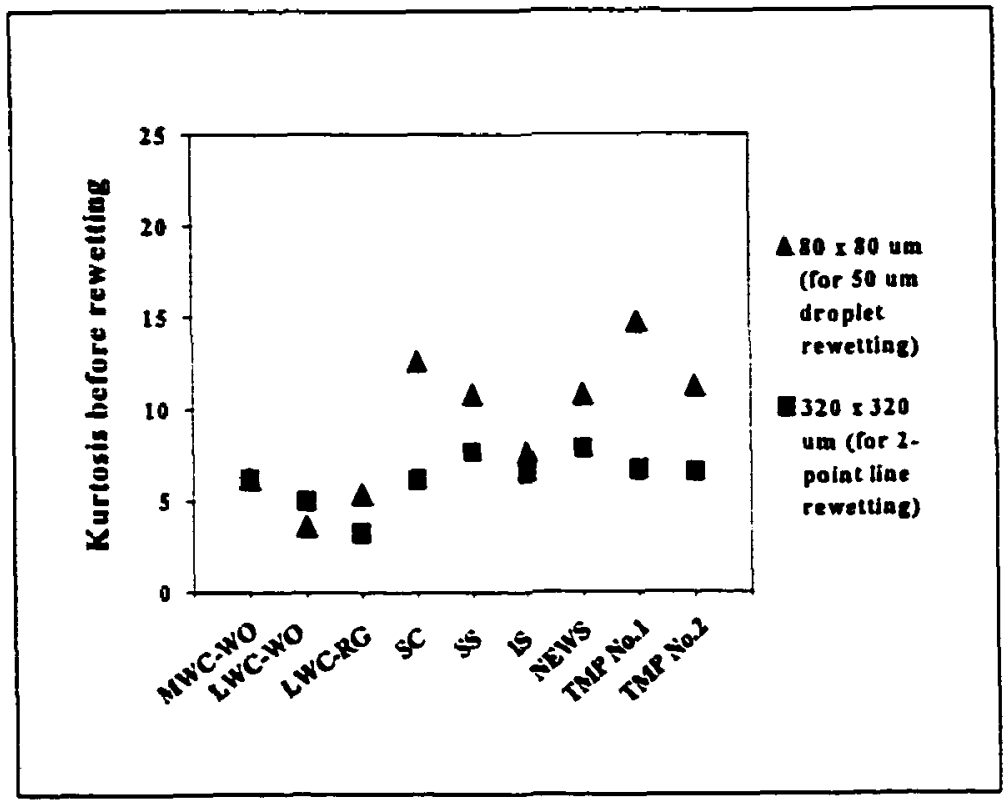

Figure 6.5. Kurtosis before rewetting vs. types of paper.

(For 2-point line rewetting, the captured area was $320 X 320 \mu \mathrm{m}$; for $50 \mathrm{~cm}$ droplet rewetting, the captured area was $80 \times 80 \mu \mathrm{m})$.

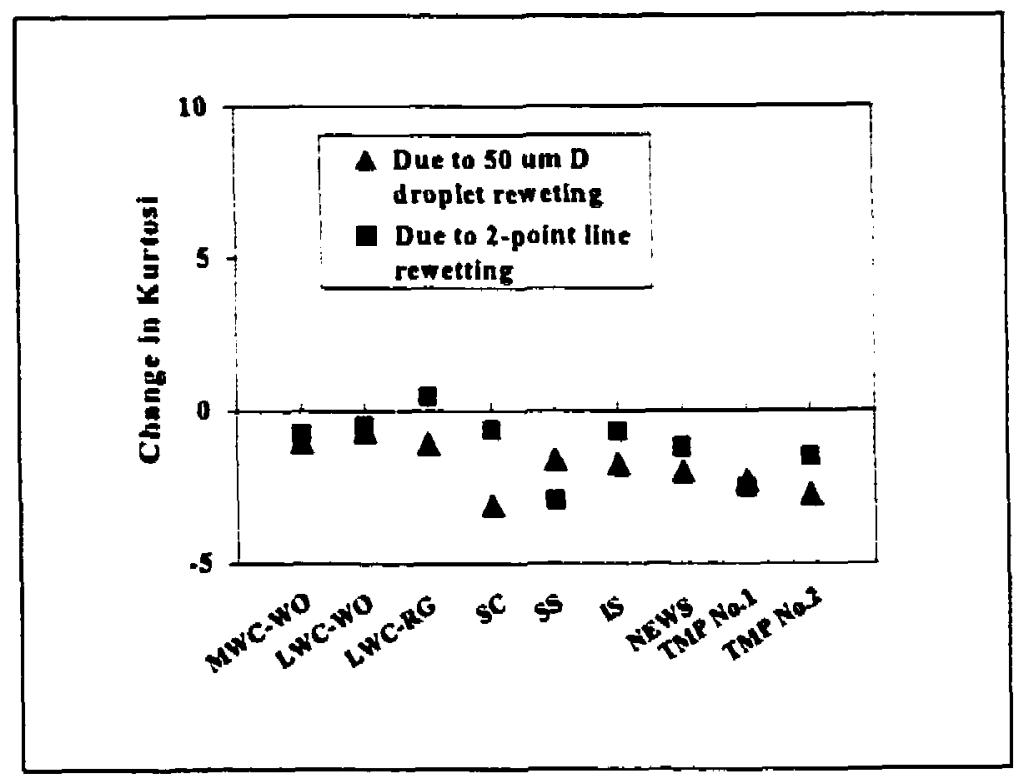

Figure 6.6. Change in kurtosis due to rewetting us. types of paper. (For 2-point line rewetting, the captured area was $320 X 320$ fm; for $50 \mu \mathrm{m} \mathrm{Ddroplet} \mathrm{rewetting,} \mathrm{the} \mathrm{captured} \mathrm{area} \mathrm{was} 80 \times 80 \mu \mathrm{m}$ ). 


\subsubsection{CHANGES IN AVERAGE STEP HEIGHT}

As described in Section 4.3.2, average step height was defined as the height difference between a selected region of the paper surface and a reference surface. During the measurement, since the reference was a stable surface, changes in average step height as a result of local rewetting represent the rising of the paper surface, i.e. local distortion in out-of-plane direction caused by local rewetting.

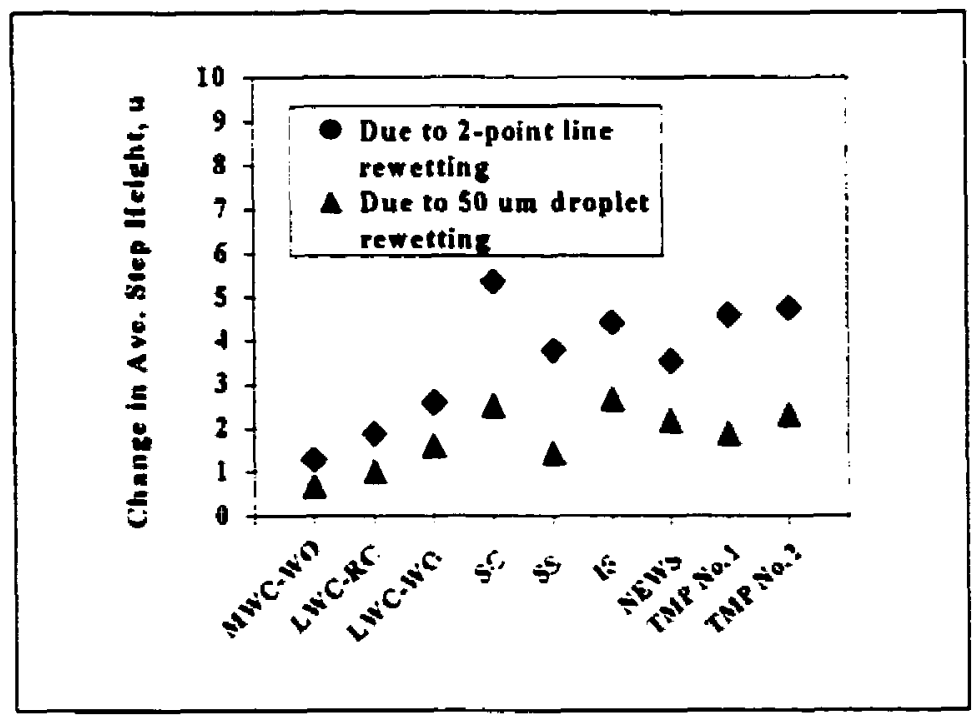

Figure 6.7. Changes in average step height vs. types of paper. (For 2-point line rewetting, the captured paper surface was320 X320 $\mathrm{\mu m}$, and the captured reference was $320 \times 160 \mathrm{~cm}$. For $50 \mathrm{~cm}$ droplet rewetting, the captured area of both captured paper surface and reference was $80 \times 80$ (m). 
Figure 6.7 indicates that the coated papers had smaller increases in average step height than uncoated paper, which means that the surface rising in coated paper was less than that in uncoated paper. As well, an increase in the amount of coating decreased the surface rising. Coating restricted the local surface rising during local rewetting.

The data also show that there was more surface rising if the paper was calendered. The supercalendered (SC) sample showed the greatest increase in average step height for both 2-point line and $50 \mu \mathrm{m}$ droplet rewetting. When water is applied to the calenderd smooth paper, the stress introduced by calendering in fiber network was released, and the wetted fibers tended to sweil to recover their natural shape. TMP No.2 was also lightly calendered paper, which is the reason that TMP No.2 changed more in average step height.

Sizing provides paper with a resistance to penetration of aqueous solutions. In this study, the sample of surface sized paper showed a relatively smaller increase in average step height for both rewetting methods as expected. The surface voids were filled by the surface sizing agents such as starch, which reduce the pore radius and therefore the rate of water penetration, leading to lesser surface rising. As well, sizing utilizes chemicals to reduce the rate of water penetration by affecting the contact angle. However, the internally sized paper sample did not show obvious evidence of resistance to water penetration. Testing proved that the internally sized paper had 
stronger wettability: $1.5 \mathrm{~mm}$ water droplets were put on both samples; the droplet on the internally sized paper disappeared immediately because of diffusion while the droplet on the surface sized paper was retained for about 10 minutes.

The change in roughness was not proportional to the change in average step height. For example, comparing Figure 6.7 to Figure 6.1 and 6.2 , the supercalendered paper has the largest values of the changes in average step height while it showed moderate changes in roughness. The changes in roughness would not represent the real situation of local distortion in out-of-plane direction. In other words, the changes in average step height expresses more accurately the local distortions in out-of-plane caused by local rewetting since the paper surface may remain flat with a small change in roughness while it is actually raised by rewetting.

Table 6.2. Effect of using different wetting liquids on the changes of average step height as well as roughness.

\begin{tabular}{|l|c|l|c|}
\hline Item & Chg. in Avg. step Ht., $\mu \mathrm{m}$ & Chg. of $\mathrm{R}_{\mathrm{a},} \mu \mathrm{m}$ & Chg. in $\mathrm{R}_{\mathbf{q},} \mu \mathrm{m}$ \\
\hline Ink-dyed water & $1.29 \pm 0.67$ & $0.34 \pm 0.18$ & $0.43 \pm 0.22$ \\
\hline Pure water & $1.36 \pm 0.37$ & $0.40 \pm 0.19$ & $0.31 \pm 0.51$ \\
\hline
\end{tabular}

Note: 2-point line rewetting: captured paper surface is $320 X 320 \mu \mathrm{m}$, and the captured reference was $320 \times 160 \mu \mathrm{m}$; sample: MWC-WO 
Ink-dyed water was used as wetting liquid for the investigation of the local distortions since the wetted area could not be distinguished when using pure water as wetting liquid (refer to Section 4.5.2). However, there are small pigment particles existing in the ink, which might affect the measurements. To investigate this possibility, pure water was used for 2-point line rewetting on the samples of MWCWO. Fortunately, at $95 \%$ confidence level, there was no significant difference between ink-dyed water and pure water. Table 6.2 is a table of experimental results. A similar test was conducted with TMP No. 2 using transparent tape as a reference (before the metal shim procedure was introduced), and there was no evidence that there was difference between ink-dyed water and pure water.

\subsubsection{EFFECT OF AMOUNT OF WATER}

It was assumed that larger amounts of water applied to paper during local rewetting would lead to greater local distortions. Three samples were tested to investigate the effect of water volume on the local distortions: medium weight coated paper (MWC-WO), supercalendered paper (SC), newsprint. Experiments were carried out with the method described in Chapter Four: printing 2 times with the 2-point line at the same location on the specimen during rewetting. For one-time printing, the amount of water applied to paper was $10.22 \mathrm{~g} / \mathrm{m}^{2}$; for two-time printing it was 20.44 $\mathrm{g} / \mathrm{m}^{2}$ (refer to Section 4.5.2). 
Table 6.3 contains the experimental results. Compared to one-time rewetting of 2-point line printing, two-time printing brought about a greater increase in the arithmetic average roughness, root means roughness, and average step height. But ttest results indicated that these increases were not significant at the $95 \%$ confidence level, except for the changes in arithmetic average roughness $\left(R_{a}\right)$ of $S C$.

Table 6.3. Results of local distortions caused by the different amount of water applied to paper. 2-point line rewetting, captured area: $320 \times 320 \mathrm{\mu m}$.

\begin{tabular}{|l|c|c|c|c|}
\hline Sample & MWC-WO & SC & News & Remark \\
\hline Change. in & $1.29 \pm 0.67$ & $5.34 \pm 1.16$ & $3.54 \pm 1.63$ & $*$ \\
Avg. step Ht., $\mu \mathrm{m}$ & $1.66 \pm 0.42$ & $6.19 \pm 2.15$ & $4.66 \pm 0.64$ & $* *$ \\
\hline Change in & $0.34 \pm 0.18$ & $1.03 \pm 0.27$ & $0.97 \pm 0.34$ & $*$ \\
$\mathrm{R}_{\mathrm{a}, ~} \mu \mathrm{m}$ & $0.42 \pm 0.17$ & $1.43 \pm 0.27$ & $1.29 \pm 0.27$ & $* *$ \\
\hline Change in & $0.43 \pm 0.23$ & $1.24 \pm 0.27$ & $1.26 \pm 0.50$ & $*$ \\
$\mathrm{R}_{\mathbf{q},}, \mu \mathrm{m}$ & $0.54 \pm 0.20$ & $1.69 \pm 0.49$ & $1.55 \pm 0.52$ & $* *$ \\
\hline
\end{tabular}

Note: * : Printing one time during rewetting. **:Printing two times during rewetting.

The amount of water of a $50 \mu \mathrm{m}$ droplet is $0.0416 \mu \mathrm{g}$ (refer to Section 4.5.2). However, there is an impact force when a water drop contacts the paper surface, and water tends to diffuse to the surrounding area. Therefore, a $50 \mu \mathrm{m}$ droplet from an ink cartridge produces a dot on paper with a diameter larger than $50 \mu \mathrm{m}$. 
Furthermore, the size of the dot is different on different grades of paper. This means that the amount of water absorbed by unit paper surface area varies from paper to paper. Using a microscope with a micrometer, experiment was conducted to examine the size of dots produced with a $50 \mu \mathrm{m}$ ink-dyed water droplet on different grades of paper.

Table 6.t. Size of dots of ink-dyed water (font I) on different grades of paper and the amount of water absorbed by paper as well as the water spreading coefficient.

\begin{tabular}{|l|c|c|c|}
\hline Sample & $\begin{array}{c}\text { Size of dot } \\
\text { Diameter, (D) } \mu \mathrm{m}\end{array}$ & $\begin{array}{c}\text { Water absorbed } \\
\text { W, } g / \mathrm{m}^{2}\end{array}$ & $\begin{array}{c}\text { Water spreading } \\
\text { coefficient, (C) }\end{array}$ \\
\hline Oiled CB & 80 & 8.28 & 0.3906 \\
MWC-WO & 172 & 1.80 & 0.0847 \\
LWC-WO & 126 & 3.34 & 0.1576 \\
LWC-RG & 126 & 1.50 & 0.0708 \\
SC & 151 & 2.32 & 0.1095 \\
News & 151 & 2.32 & 0.1095 \\
TMP No.t & 147 & 2.45 & 0.1156 \\
TMP No.2 & 144 & 2.55 & 0.1204 \\
SS & 106 & 4.72 & 0.2228 \\
IS & 130 & 3.14 & 0.1482 \\
\hline
\end{tabular}


Table 6.4 lists the experimental results and the corresponding values of the amount of water absorbed by unit paper area as well as the water spreading coefficient. The water spreading coefficient is defined as:

$$
C=D_{0}^{2} / D^{2}
$$

where $D_{0}$ is the diameter of ink-dyed water droplet of font $1(50 \mu \mathrm{m})$,

$D$ is the diameter of dot produced with the $50 \mu \mathrm{m}$ ink-dyed water droplet.

The amount of water absorbed by per unit paper area is calculated according to the following equation:

$$
W=C \times 0.0416 \times 10^{-6} / \pi(D / 2)^{2}
$$

The values $(\mathrm{W})$ of the amount of water in table 6.4 may not be accurate since the wetting rate and evaporation of water are neglected, and these factors relate to mainly the papermaking processes such as sizing and coating. Figure 6.8 shows the relationship between the changes in average step height and water spreading coefficient for $50 \mu \mathrm{m}$ droplet rewetting. Generally speaking, for uncoated samples, the changes in average step height decreased with the increase in water spreading coefficient. 


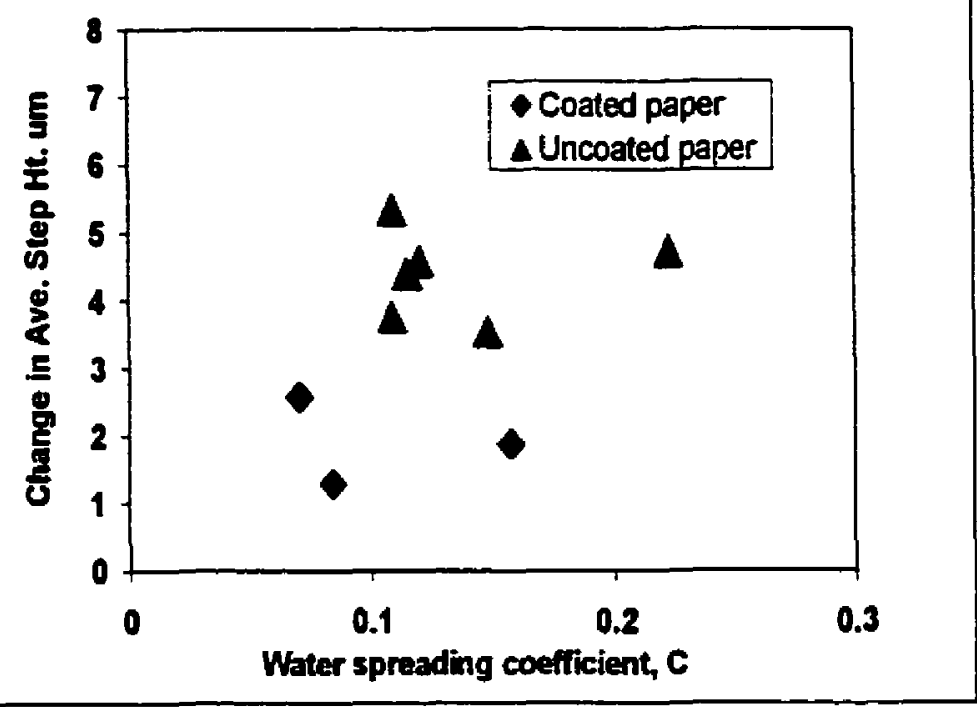

Figure 6.8. Changes in average step height vs. water spreading coefficient. (50 um droplet rewetting).

\subsection{EFFECTS OF PAPER CHARACTERISTICS}

\subsubsection{ROUGHNESS}

The original roughness of the paper surface influences water absorption, consequently affecting the local dimensional stability of paper structure. Figure 6.9 and 6.10 provide information about the relationship between the changes in average step height and arithmetic average initial roughness. As indicated in Figure 6.9, calendered samples (including supercalendered paper (SC) and lightly calendered 


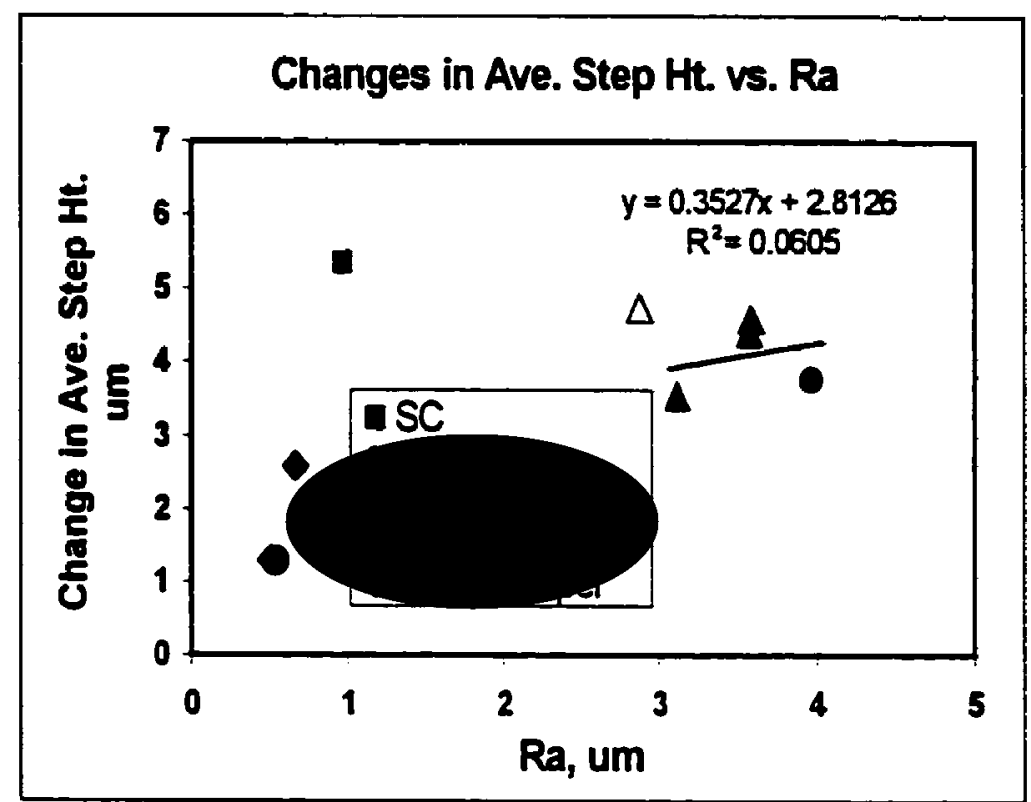

Figure 6.9. Cahnges in average step height vs. Ra. (2-point line printing rewetting; uncoated paper: IS, newsprint. TMP No.I)

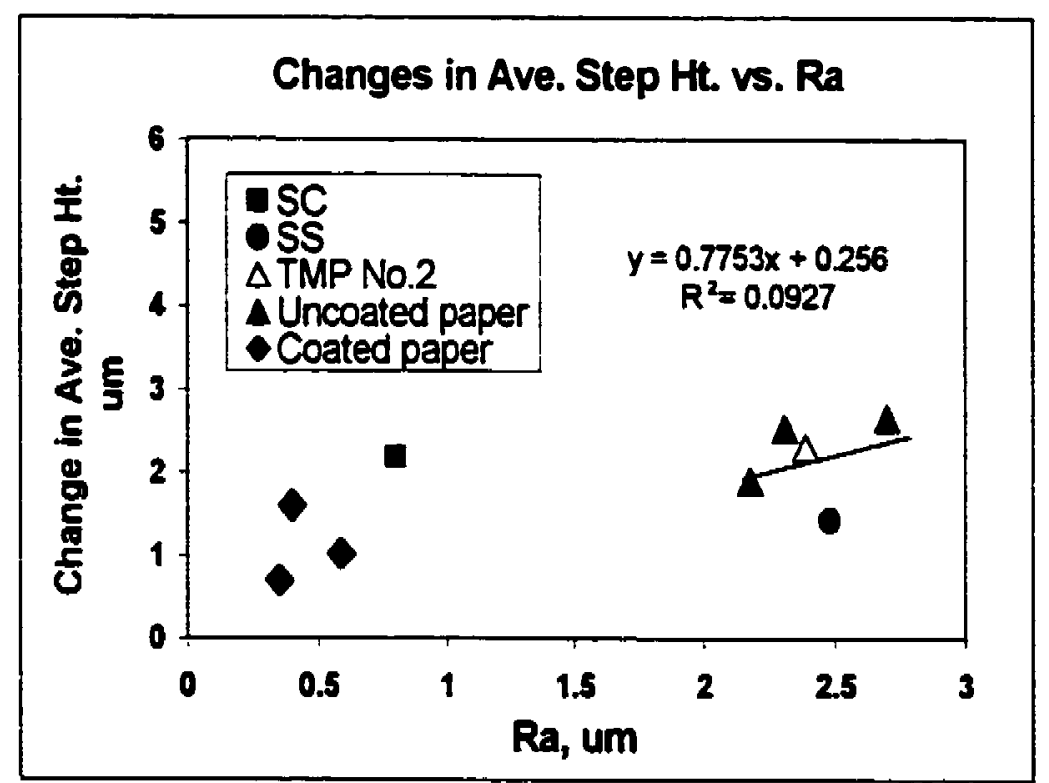

Figure 6.10. Changes in average step height vs.Ra. (50 $\mu \mathrm{m}$ droplet rewetting; uncoated paper: $[S$, newsprint, TMP No.I) 
paper (TMP No.2)) showed larger changes in average step height even though the SC had a smooth initial surface with a smaller value of $R_{a}$ As well, the surface sized paper (SS) presented smaller change in average step height even though its surface was initially rougher. The correlations between the increases in average step height and $\mathbf{R}_{\mathrm{a}}$ are weak. However, for both rewetting methods, there is an increasing tendency in the changes in average step height with the increase of $R_{a}$ if SC and TMP No.2, are considered as special examples.

\subsubsection{BOND STRENGTH}

Bond strength was used in this study as a parameter to describe the degree of fiber-tofiber bonding. It is characterized by the delamination energy of paper, which is defined in Section 5.5. Figure 6.11 and 6.12 present the relationships between the changes in average step height and bond strength. For both rewetting methods, with an increase of bond strength, the changes in average step height were reduced. As introduced in Section 5.5, since the sizing agents such as starch and rosin might have an affinity for fibers and were sorbed onto them, strengthening fiber-to-fiber bonding, and therefore the sized papers had relatively greater bond strength. In addition, the sizing pigments filled the paper voids, providing a resistance to penetration of water. As a result, the two sized copy papers showed a relatively smaller increase in average 


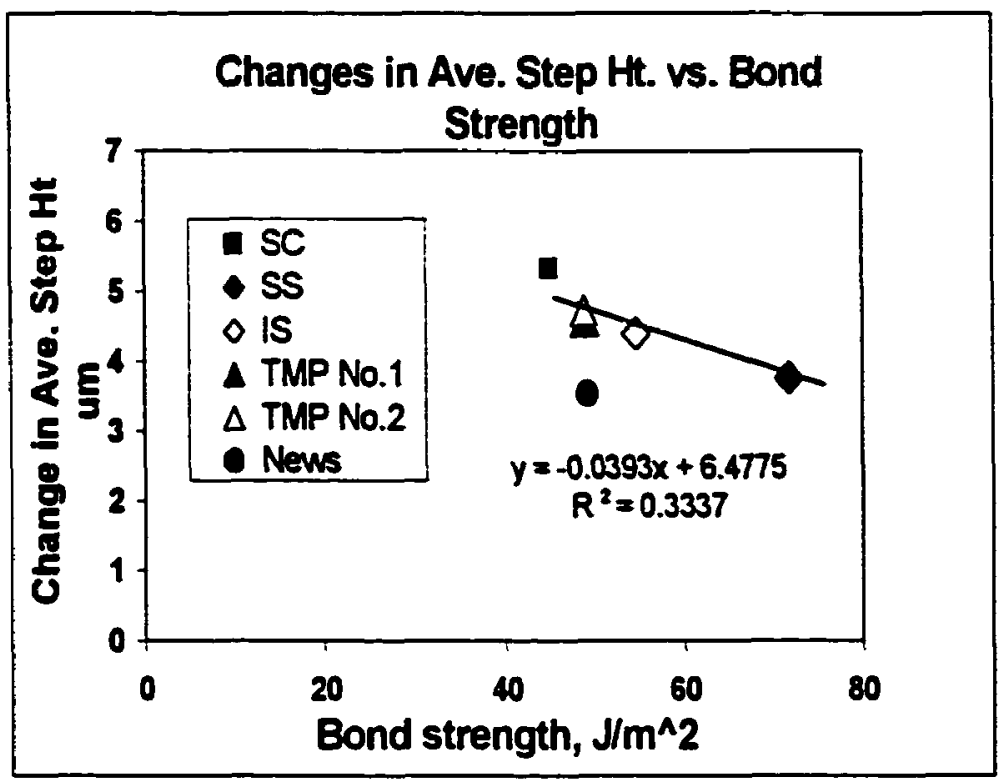

Figure 6.11. Changes in average step height vs. bond strength. (2-point line printing rewetting)

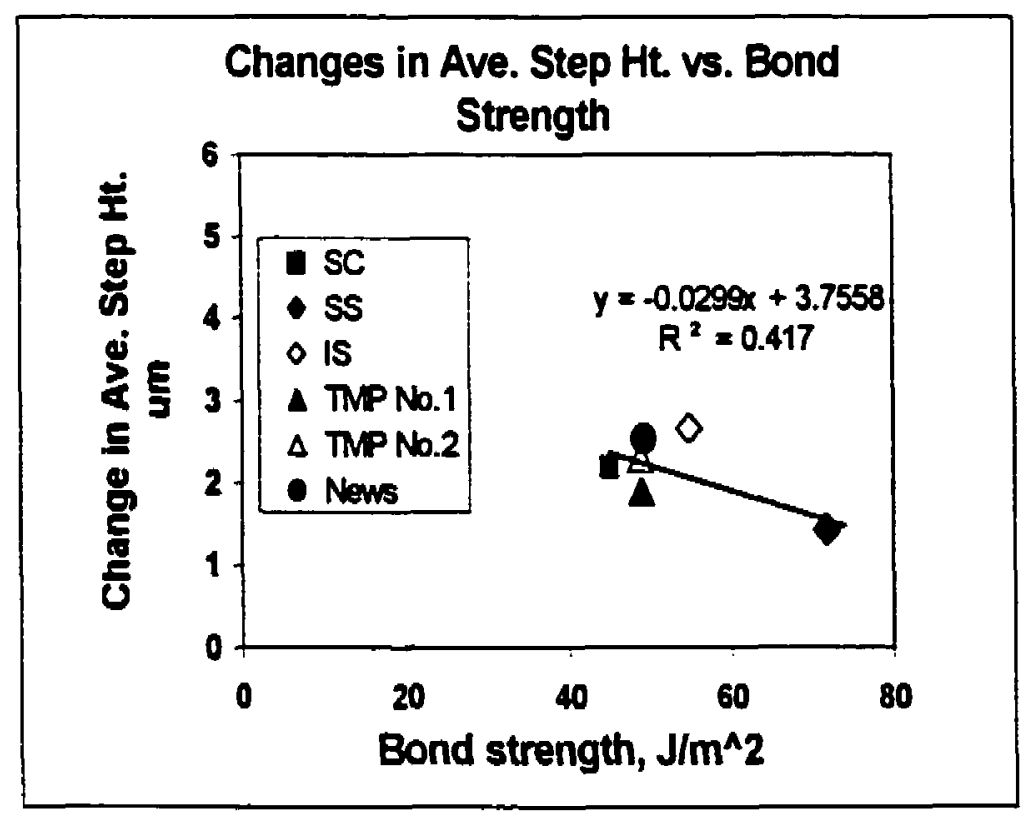

Figure 6.12. Changes in average step height vs. bond strength. (50 $\mu \mathrm{m}$ droplet rewetting). 
step height with a higher value of bond strength, especially for surface sized copy paper. As Figure 6.11 and 6.12 show, comparing with sized copy paper, TMP No.1, and TMP No.2 presented smaller bond strength and greater changes in average step height. Copy papers are usually made of chemical pulps. This suggests that the distortions caused by rewetting may be affected by the content of mechanical pulps. It was hypothesized that the sample of newsprint should increase relatively more in average step height after rewetting since newsprint is made of mechanical pulps. But for 2-point line rewetting, the experiments did not show this phenomena.

\subsubsection{DENSITY}

As Figure 6.13 and 6.14 indicate, for uncoated samples except the supercalendered paper (SC), the increases in average step height were reduced with the increase of sheet density even though the tendency and correlation are weak for the case of $\mathbf{5 0}$ $\mu \mathrm{m}$ droplet rewetting. This suggests that, to certain degree, an increase in sheet density means individual fibers bonded with more surrounding fibers, increasing bond strength and leading to fewer fiber rising.

Sizing increases the sheet density with sizing agents of high density, which reduces the contact area between fibers; however, it enhances the bonds since sizing agents have an affinity for fibers, leading to stronger bond strength. In addition, sizing 


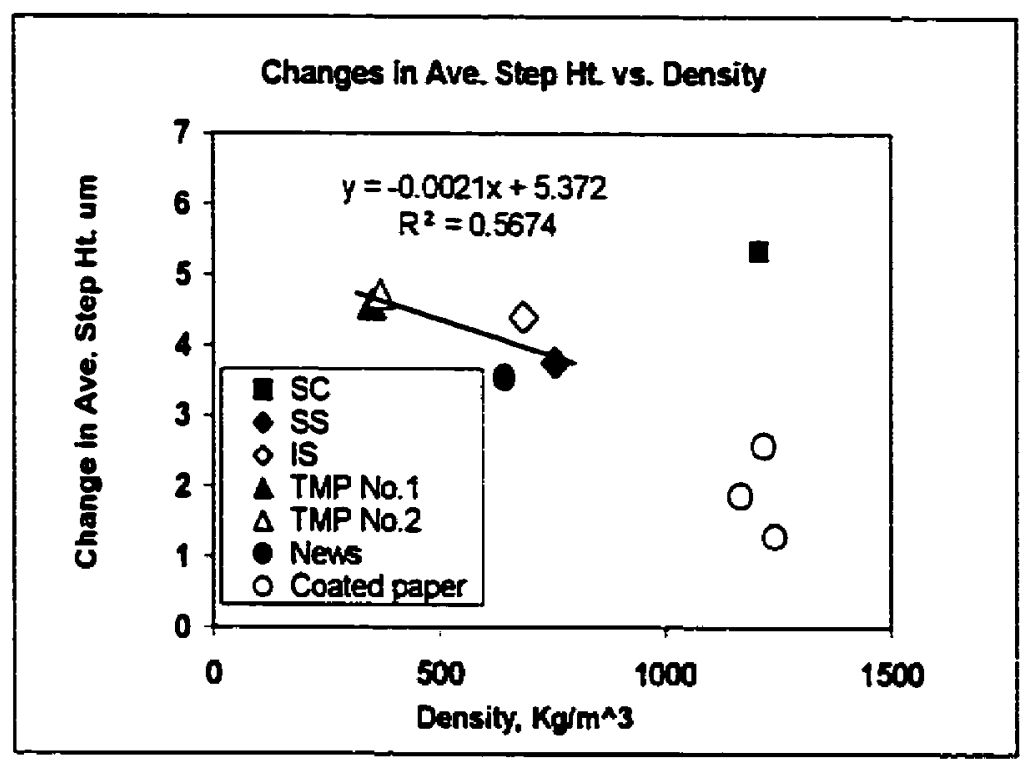

Figure 6.13. Changes in average step height vs. paper density. (2-point line printing rewetting).

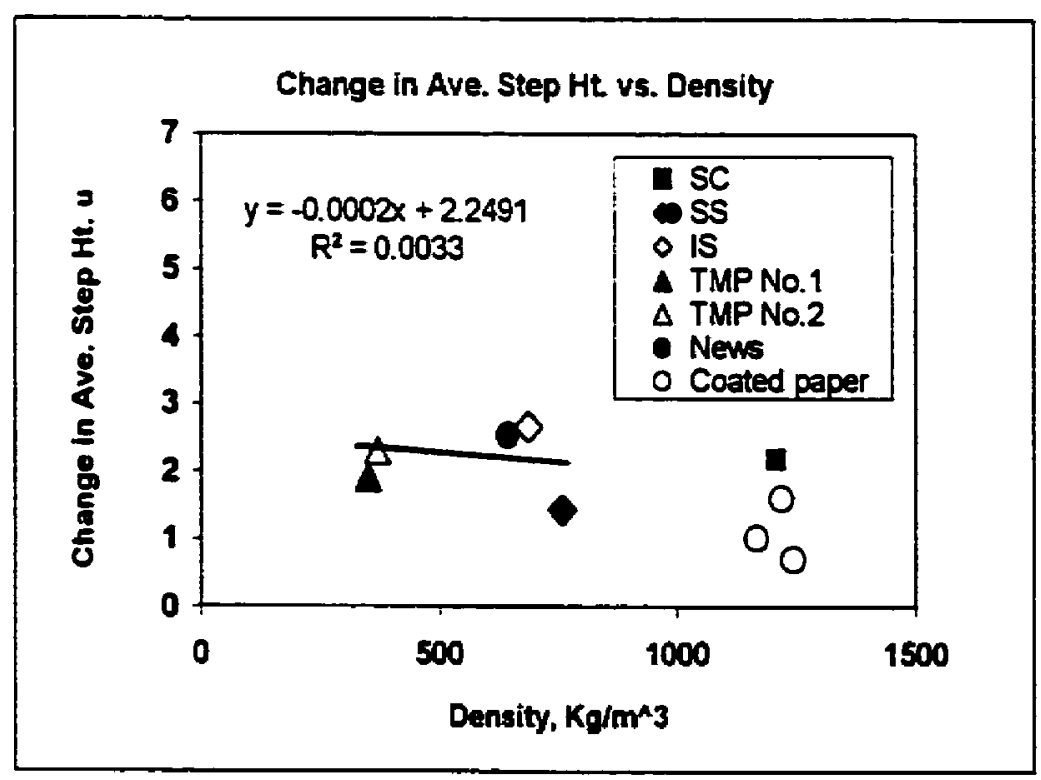

Figure 6.14. Changes in average step height vs. paper density. (50 $\mu \mathrm{m}$ droplet rewetting). 
reduces the sheet porosity, providing paper a resistance to the penetration of water. Therefore, the two sized papers showed relatively small increases in average step height. The supercalendered paper (SC) represents a special example. The density of the $\mathrm{SC}$ was the greatest while it increased in average step height. The supercalendered paper represents a special example. The density of the SC was the greatest while it increased in average step height while also the greatest value for 2-point line rewetting. This is because that the internal stress was released in the fiber network and the collapsed fibers tended to recover their original tube-shape when they contacted water. Supercalendering may also reduce the bond strength even though the sheet density was increased. Due to the high calendering pressure the fibers collapsed and themselves were densified, leading to reduction of space between fibers and consequent decrease in bond strength.

The statistical parameters were strongly dependent on the information of the local paper surface. The selected area of paper surface for $50 \mu \mathrm{m}$ droplet rewetting was only $80 \times 80 \mu \mathrm{m}$. For uncoated paper, the variability of basis weight, i.e., formation, changes from one location to another. This may be the reason that the correlation between the increase in average step height and paper density is a weak. 


\section{CHAPTER SEVEN}

\section{MODELLING THE CHANGE IN SURFACE HEIGHT}

\subsection{INTRODUCTION}

Through the experimental investigation introduced in the previous chapters, a preliminary understanding of the phenomenon of local distortions due to local rewetting has been obtained. Based on the experimental results and the knowledge of paper characteristics, a model has been developed for the prediction of the local distortions resulted from local rewetting. In this model, the local distortion is characterized by the changes in average step height, i.e., local surface height. The amount of water applied to paper and relevant paper properties including arithmetic average roughness $\left(R_{a}\right)$, bond strength, and sheet density are taken into account as the variables that affect distortion. Even though it is an empirical equation, the model values show a good agreement with the experimental results.

\subsection{VARIABLES}

It is accepted that the more water applied to paper and the greater water absorbency of the paper, the more tiber rising is expected [6]. The experimental 
results in this study (refer to Section 6.5) also showed an increasing tendency for the changes in average step height when increasing the amount of water.

Rough paper can retain more water than smooth paper when water contacts the paper surface $[16,21,23]$, and therefore a rough surface increases fiber rising and sheet roughening. Experimental results indicate that there is an increasing tendency in the changes in surface height when paper roughness increases (If SC and TMP No.2 are considered as special examples. Refer to Figure 6.8 and 6.9). Therefore, it was hypothesized that the initial roughness of the local paper surface to be wetted would be one of the factors that have influence on the local distortions.

Paper is a network of bonded fibers. It has been reported that the average millimeter length of fiber contacts with as many as 20 to 40 other fibers [60]. Fiberto-fiber bonds play an important role in the behavior of paper surface during the application of aqueous liquids to paper in coating and printing [50]. In this study, the degree of fiber-to-fiber bonding is characterized by the bond strength, which is related to the delamination energy per unit area of paper. As indicated in Figure 6.11 and 6.12 , the changes in average step height due to rewetting decrease with increases of bond strength. This is reasonable because more bond strength means the paper has greater ability to resist fiber rising, reducing the increases in surface height. Thus, bond strength would be a variable negatively affecting the surface rising. 
Bond strength does not fully describe the total bonding degree of fibers in the paper sample. The parameter of bond strength employed in this study is an average value representing the bonding degree of fibers in the delamination interface. In order to better represent the lateral bonding degree through the sheet thickness, sheet density is also included in the model. For uncoated papers, a denser sheet means greater bonding both in in-plane and out-of-plane directions. In this study, the changes in average step height were plotted against the sheet density in Figure 6.13 and 6.14. These figures show reduction in the changes in average step height with an increase in sheet density.

\subsection{ESTABLISHMENT OF THE MODEL}

Based on the analysis in the previous section, an equation can be written to describe the relationship between the increases in average step height and the amount of water applied to paper and the paper properties:

$$
\Delta H=f\left(R_{a}, W, T_{b}, \rho\right)
$$

where $\Delta H$ is the increase of average step height, $\mu \mathrm{m}$

$R_{a}$ is arithmetic average roughness, $\mu \mathrm{m}$,

$W$ is the amount of water applied to paper, $\mathrm{kg} / \mathrm{m}^{2}$, 
$T_{b}$ is fiber-to-fiber bonding strength, $\mathrm{J} / \mathrm{m}^{2}$,

$\rho$ is paper density, $\mathrm{kg} / \mathrm{m}^{3}$.

Since the increases in average step height $(\Delta \mathrm{H})$ showed an increasing tendency with an increase of $R_{a}$ or $W$ while $\Delta H$ decreased when $T_{b}$ or $\rho$ increased according to the experimental results, equation 7.1 can be re-written as:

$$
\Delta H=k R_{a}^{\prime} W^{*} T b^{\circ} \rho^{\prime}
$$

where, $k$ is $a$ constant (dimensionless); $1, \mathrm{~m}, 0$, and $\mathrm{p}$ are exponents. $\mathrm{I}$ and $\mathrm{m}$ are positive; $o$ and $p$ are negative. By means of physical analysis described above as well as dimensional analysis, a model was obtained:

$$
\Delta H=k \frac{R_{a} W}{\sqrt{\left(\frac{T_{b}}{g}\right) \rho}}
$$

where, $g$ is acceleration due to gravity, $\mathrm{m} / \mathrm{s}^{2}$.

The $W$ is the amount of water applied to paper. For 2-point line rewetting, the value of $W$ is $10.22 \mathrm{~g} / \mathrm{m}^{2}$. For $50 \mu \mathrm{m}$ droplet (font $l \mathrm{dot}$ ) rewetting, referring to Table 6.4 in Section 6.2.4, we have: 
where $C$ is the water spreading coefficient, dimensionless,

$D$ is the diameter of dots (font 1 ) on different grades of paper, $\mu \mathrm{m}$,

$W_{0}$ is the water volume of a $50 \mu \mathrm{m}$ droplet, $0.0416 \mu \mathrm{g}$.

\subsection{VERIFICATION}

Equation 7.3 relates the local surface rising in terms of the changes in average step height caused by local rewetting to four variables. The verification of this equation 7.3 ideally should be implemented by systematically varying one variable and determining its effects on the average step height while keeping all other variables constant. However, it was impossible to do this because of the limitations of our experimental methodology. Instead, while measuring the changes in average step height, all of the parameters used in equation 7.3 as independent variables were obtained for the uncoated samples including TMP No.1, TMP No.2, newsprint, surface sized copy paper, and internally sized copy paper. Based on the data of the $R_{a} W, T_{b}$ and $p$, a model value can be calculated using equation 7.3 .

The value of constant $k$ in equation 7.3 was estimated by using one set of the test data. For example, based on the experimental results $\left(\Delta H, R_{a} W, T_{b}, \rho\right)$ of the 


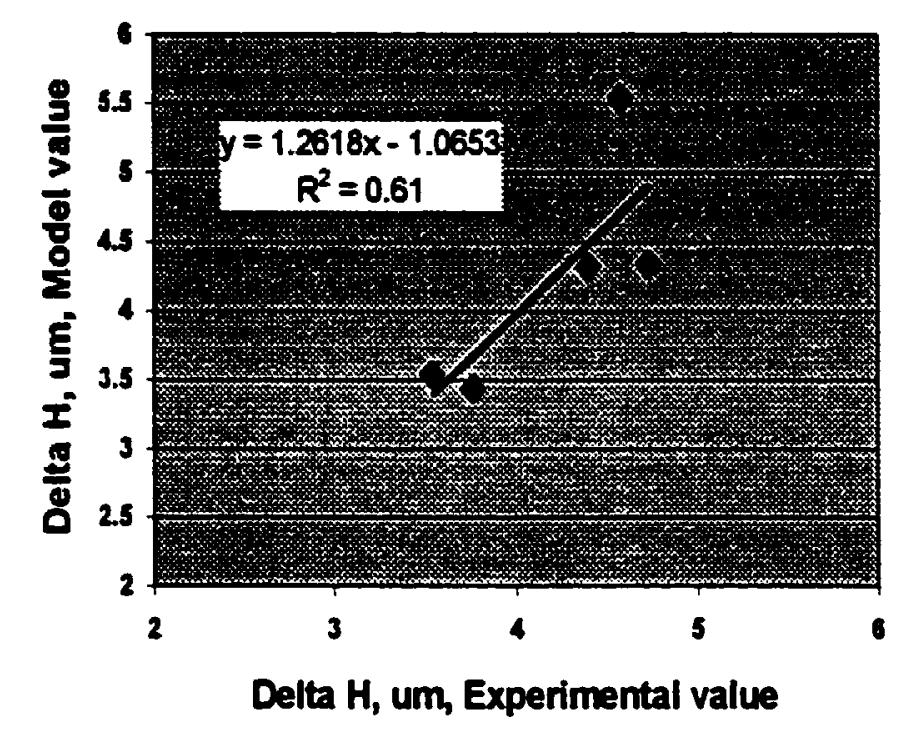

Figure 7.1. Correlation between model values and experimental results. (2-point line rewetting).

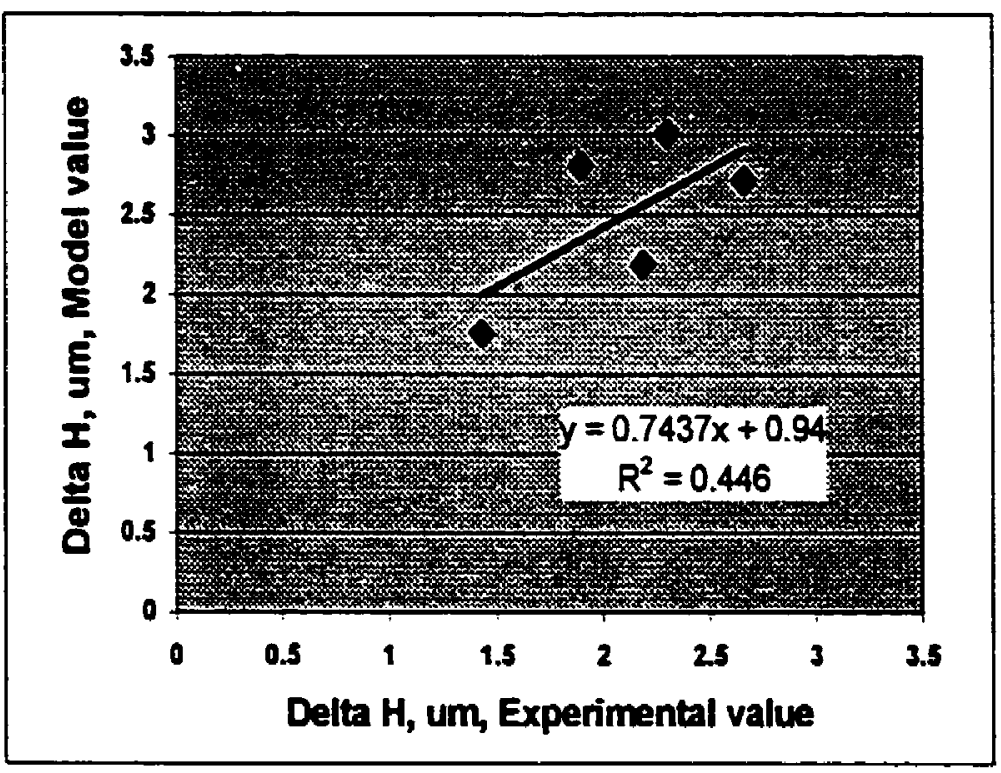

Figure 7.2. Correlation between model values and experimental results. (50 ym D droplet rewetting) 
newsprint sample, the value $k$ for 2-point line rewetting is $6.319 \mathrm{E}+3$, and for $50 \mu \mathrm{m}$ droplet rewetting it is $2.545 \mathrm{E}+3$.

As Figure 7.1 and Figure 7.2 indicate, there is good agreement between model values and experimental results for both rewetting methods. However, the model values of $\Delta H$ were calculated by assuming that the value of $\mathrm{C}$ (water spreading coefficient) equals 1 . Different values of the $C$ are listed in Table 6.5 for different grades of paper, but the correlation between the model values and the experimental result is very weak. Perhaps $C$ (water spreading coefficient) cannot represent the real spreading ability or the wettability of the paper samples since the absorbency of water in paper involves many factors such different agents added during coating and sizing, which affects the contact angle and therefore influences the wettability. 


\section{CHAPTER EIGHT}

\section{CONCLUSIONS AND RECOMMENDATIONS}

\subsection{CONCLUSIONS}

(l) There is good agreement among WYKO arithmetic average roughness, PPS roughness, and the roughness measured using a mechanical stylus profilometer. The WYKO ${ }^{\text {TM }}$ NT 2000 system proved to be an excellent experimental apparatus, which can be applied to the investigation of the changes in paper surface structure.

(2) Local rewetting leads to local roughening, i.e., an increase in local roughness including arithmetic average roughness $\left(\mathbf{R}_{\mathrm{a}}\right)$ and root mean square roughness $\left(R_{q}\right)$. $R_{q}$ increases more than $R_{a}$ after rewetting, suggesting that more bumps and valleys dominate the surface profile as a result of rewetting.

(3) Due to local rewetting, the skewness $\left(R_{\text {sij }}\right)$ increases while the kurtosis $\left(\mathbf{R}_{\mathrm{ku}}\right)$ decreases, which means that rewetting bring about more peaks rather valleys while these peaks and valley are more randomly 
distributed.

(4) An increase in local surface height does mean a corresponding increase in roughness. A paper surface may be totally raised by local rewetting and consequently fiber swelling, keeping the surface roughness with a small change.

(5) Dapcimakinīy piiuciesses such as coating, sizing, and calendering affect the local distortions caused by rewetting. Coating and sizing provide paper with a resistance to penetration of water, leading to lesser local distortions. For calendered papers, because of rewetting the internal stress introduced by calendering pressure is released, and the collapsed fibers tends to recover their natural tube-shape, resulting in significant local distortions.

(6) The amount of water applied to paper, initial local surface roughness, fiber-to-fiber bond strength, and sheet density play important roles in the local paper surface rising when paper contacts water. More water results in greater local surface rising. Rough paper surfaces possess stronger absorbency of water, leading to greater surface rising. Fiberto-fiber bonding restricts the fibers from rising during rewetting, and increasing sheet density helps to increase the degree of bonding 
between fibers, reducing the surface rising tendency.

(7) The proposed empirical model relates the local surface rising to the amount of water applied to paper and the paper properties in terms of arithmetic average roughness, fiber-to-fiber bond strength, and sheet density. Model predictions agree well with the experimental results. The development of this model reveals the relationship between the fundamental factors that affect the local distortions due to local rewetting.

\subsection{RECOMMENDATIONS}

(1) The paper samples used in this study were provided by the industry, and general information about the paper properties was available but without details about specification of the raw materials, process procedure, and fillers and chemicals added, etc. Therefore, it was difficult to analyse all the factors that could affect the distortions caused by water. For further work, the samples should be chosen with detailed information such as the content of mechanical pulp, percentage of filler, and the types and amount of chemicals added. 
(2) In this study, the rewetting of samples was not conducted in situ. During measurement, the sample had to be taken off the WYKO sample stage and inserted into the slot of the modified printer for rewetting. With the help of a microstage and a fixed clamp, it was easy to relocate the samples. Images could be taken before and after rewetting at the same position on the sample. However, a device which allows one to rewet the samples in situ will be helpful to eliminate the operator error during sample relocation which may occur while using the inkjet printer.

(3) The properties chosen as variables in equation 7.3 are local surface roughness, bond strength, and sheet density. Since the bond strength and sheet density are the average values for the whole sheet, they may not represent the local values of these variables. Better parameters, which represent the characteristics of the fibers and their network in the local region, should be considered.

(4) Because of the limitations of our experimental methodology, the proposed model could not be verified systematically. More work is needed for the verification of the model. 


\section{REFERENCE}

[1] J. Skowrongski and P. Lepoutre, water-paper interaction during paper coating, Tappi Journal, Vol.68, No.11, p.98-102, November, 1985.

[2] P. O. Trollsas, Water uptake in newsprint during offset printing, Tappi Journal, Vol.78, No.1, p.155-160, January, 1995.

[3] W. Gallay, Stability of Dimensions and form of paper, Tappi Journal, Vol.56, No.11, p.54-63, November, 1973.

[4] Y. Nanri and T. Uesaka, Dimensional stability of mechanical pulps-drying shrinkage and hygroexpansity, Tappi Journal, Vol.76, No.6, p.62-66, June, 1993.

[5] N. Gurnagul and D.G. Gary, The response of paper sheet surface areas to changes in relative humidity, Journal of Pulp and Paper Science, Vol.13, No.5, p. 159-164, September, 1987.

[6] J. S. Aspler and M.-C. Beland, A review of fiber rising and surface roughening effects in paper, Journal of Pulp and Paper Science, Vol.20, No.1, p.27-32, January, 1994.

[7] J. F. Waterhouse, The mechanical and physical properties of paper surfaces, Chapter 3, Surface analysis of paper, Edited by Terrance $E$. Conners, Sujit Banerjee, 1995, CRC Press Inc.

[8] K. Niskanen, Paper Physics, 1998, Published by Faper Oy. 
[9] M. Deng and C.T.J. Dodson, Paper - An engineered stochastic structure, Tappi Press, Átianta, ÚSÁ, i994.

[10] C. T. J. Dodson, A universal law of formation, Journal of Pulp and Paper Science, Vol.16, No.4, p.136-137, July, 1990.

[II] J. F. Waterhouse, The effect of some papermaking variable on formation, Tappi Journal, Vol.76, No.9, p. 129-134, September, 1993

[12] W.H. Bureau, Paper - from pulp to printing, Graphic Arts Publishing Co., Chicago, 1968.

[13] A. Wu, M.T. Kortschot, Y. Chen, and R. Kong, Variability in the local structure and properties of paper, Tappi Internal Paper Physics Conference, p. $155-159,1999$.

[14] W. R. Haselton, The application of gas adsorption techqiques to the study of the area and structure of pulps and the unbonded and bonded area of paper, Tappi Journal, Vol. 38, No.8, p.716, 1955.

[15] J.W. Swanson and A.J. Steber, Fiber surface area and bonded area, Tappi Journal, Vol. 42, No.12, P986, 1955.

[16] M. Hoc, Fiber rising in papers containing mechanical pulp, Tappi Journal, Vol. 72, No.4, p.165-169, 1989.

[17] J. Skowronski, P. Lepoutre, and Bichard, Measuring the swelling pressure of paper, Tappi Journal, Vol. 71, No.7, p.125-129, July, 1988. 
[18] AM. Scallan, Fiber-water interactions in papermaking, Edited by W. Cloves, Londom, 1978.

[19] H.W. Emerton, Handbook of paper science, Vol. 1, Edited by Elsevier, Amsterdam, 1980.

[20] J. Berthod, J. Desbrieres, M. Rinaudo, and L. Salmen, Types of adsorbed water in relation to the ionic groups and their counterions for some cellulose derivatives, Polymer, Vol. 35, No. 26, p.5729-5736, December, 1994.

[21] P. O. Trollsas, Water uptake in newsprint during offset printing, Tappi Journal, Vol. 78, No. I, p. 155-160, January, 1989.

[22] M. C. Beland, N. Nguyen, and G. de Silviera, New image analysis for fiberrising measurement, Journal of Pulp and Paper Science: Vol. 19 No.3, P.114-118, May, 1993.

[23] T. Forseth and T. Helle, Moisture-Induced Roughening During "Water Coating" of Precalendered wood-containing Paper, Journal of Pulp and Paper Science, Vol. 24, No. 10, p. 125-132, October, 1998.

[24] Y. Nanri and T. Uesaka, Dimensional stability of mechanical pulps drying shrinkage and hygroexpansivity, Tappi Journal, Vol.76, No.6, p.62-66, June, 1993.

[25] J. Skowronski, Surface roughening of precalendered base sheets during coating, Journal of Pulp and Paper Science: Vol. 16 No.3, p. 102-109, 1990.

[26] J. D. Peel, Paper science and paper manufacture, Edited by G. A. Smook, 
Angus world publications Inc., Vancouver, 1999.

[27] J.R. Parker, Development and application of a novel roughness tester, Tappi Journal, Vol.54, No.6, p.943-949, June, 1971.

[28] J.R. Parker, The measurement of printing roughness, Tappi Journal, Vol.64, No.12, p.56-58, June, 1981.

[29] M. C. Beland and P. J. Mangin, Three-Dimensional evaluation of paper surface using confocal microscopy, Chapter I, Surface analysis of paper, Edited by Terrance E. Conners, Sujit Banerjee, 1995, CRC Press Inc.

[30] I. M. Kajanto, Effect of formation on print quality with wood-free offset papers, Nordic Pulp Paper Research Journal, No. l, p.8-15, April, 1989.

[31] W. E. Scott and J. C. Abbott, Properties of paper: an introduction, Tappi Atlanta, Georgia, Tappi Press, 1995.

[32] I. H. Parker, S. Wei, and Y. Wen, Effect of pore structure and sizing on absorption of liquid by paper, P349, 50 ${ }^{\text {th }}$ Appita Annual General Conference, 1996 (Appita).

[33] T. Uesaka and D.Qi, Hygroexpansivity of paper - effects of fiber-fiber bonding, Joumal of Pulp and Paper Science, Vol.20, No.6, p. 175-179, June, 1994.

[34] U. Weise and H. Paulapuro, Effect of drying and rewetting cycles on fiber swelling, Journal of Pulp and Paper Science, Vol.25, No.5, p.163-166, May, 1999. 
[35] J.E. Stone and A.M. Scallan, Effect of component removal upon the porous structure of the cell wall of wood. II. Swelling in the water and the fiber saturation point, Tappi Journal, Vol.50, No.10, p.149-459, October, 1967.

[36] M.B. Lyne and J.S. Aspir, Wetting and the sorption of water by paper under dynamic conditions, Tappi, Vol.65, p.98-101, December, 1982.

[37] P. Lepoutre, M.B. Inoue, and J. S. Aspir, Wetting time and critical surface tension, Tappi Journal, Vol.68, No.12, p.86-87, 1985.

[38] A. M. Cazabat, The dynamics of wetting, Nordic Pulp and Paper Research Journal No.2, Vol.4, No.2, p.146-150, July, 1989.

[39] J. S. Aspler, S. Davis, and M. B. Lyne, The surface chemistry of paper in relation to dynamic wetting and lithographic fountain solutions, Journal of Pulp and Paper Science, Vol. 13, No.2, p.55-60, March, 1987.

[40] T. Uesaka, Dimensional stability of paper: Upgrading paper performance in end use, Journal of Pulp and Paper Science, Vol.17, No.2, p.39-41, March, 1991.

[41] L. Salmen, C. Fellers, and M. Htun, Development and release of dried-in stress in paper, Nordic Pulp and Paper Research Journal, No.2, p.44-48, 1987.

[42] H.F. Jang, A.G. Robertson, and R.S. Seth, Optical sectioning of pulp fibers using confocal scanning laser microscopy, Tappi Journal, Vol. 74, No. 10, p.217-219, 1991 .

[43] P.J. Mangin and M. C. Beland, Three-dimensional evaluation of paper 
surfaces using confocal microscopy: Applications to research and development. In Advances in printing science and technology, Edited by W.H. Banks, ed. Pentech Press, London, Vol.22, p.159, 1994.

[44] WYKO surface profilers technical reference manual, WYKO Corporation, May 1996

[45] WYKO NT-2000 operator's guide, WYKO Corporation, Vision 1.0, January, 1997.

[46] http:www.slac.standford.edu/exp/mps/FCS/FCS-drop.htm

[47] T. Wriedt, Power and particle measurement techniques, http://www.fb4.uni-bremen.de/-fg0l/laser.htm

[48] H. Ulmke, T. Wriedt, H. Lohner, and K. Bauckhage, The piezoelectric droplet gernerator - a versatile tool for dispensing applications and calibration of particle sizing instruments, P. Mckeown et al. ed., Precision Engineering - Nano technology, Proceedings of the $1^{\text {st }}$ International euspen Conference, Vol.2, P290, Shaker Verlag, Aachen, 1999.

[49] J.F. Oliver, Initial stages of ink jet drop impaction, spreading, and wetting on paper, Tappi Joumal, Vol.67, No.10, p.90-94, 1984.

[50] J.F. Oliver and R.C. Forsyth, A dynamic liquid sorption apparatus for studying interactions of microscopic drops in situ on porous substrates, Colloids and Surfaces Vol.43, p.295-305, 1989

[51] http:www.hp.com/oeminkjet/techno/ 
[52] Hannes Vomhoff, On the in-plane permeability of water-saturated fiber webs, Nordic Pulp \& Paper, Vol.15, No.3, p.200-204, 2000

[53] J. Skowronski and W. Bichard, Fiber-to-fiber bonds in paper. Part I. Measurement of bond strength and specific bond strength, Journal of Pulp and Paper Science, Vol.13, No.5, p. 165-169, September, 1987.

[54] J. Skowronski, Fiber-to-fiber bonds in paper. Part II. Measurement of breaking energy of fiber-to-fiber bonds, Journal of Pulp and Paper Science, Vol.17, No.6, p.217-222, November, 1991.

[55] S. G. Kapoor, S.M. Wu, and S. M. Pandit, An new method for evaluating the printing smoothness of coated papers, Tappi Journal, Vol.61, No.6, p.71-74, June, 1978.

[56] M. A. MacGregor, T.E. Conners, MD microstriations in paper, a two-sided shrinkage phenomenon, Tappi Journal, Vol.72, No.4, p.177-181, April, 1989.

[57] K. Murakami, R. Imamura, and T. Yamamoto, Japan Tappi Journal, Vol.27, No.2, p.55, 1973.

[58] P. Wagberg and $P$. Johanson, Surface profilometry - a comparison between optical and mechanical sensing on printing papers, Tappi Journal, Vol.76, No.12, p.I15-121, December, 1993.

[59] T. Enomae and P. LePoutre, Stylus profilometry on paper. marking by the stylus, Tappi Journal, Vol. 78, No.10, p.173-176, October, 1995. 
[60] D. H. Page, A theory for the tensile strength of paper, Tappi Journal, Vol.54, No.4, p.674-681, April, 1969. 
Appendix 1. Roughness $\left(R_{\mathrm{a}}, \mu \mathrm{m}\right)$ measured using WYKO ${ }^{\text {tw }}$ NT-2000 system.

Experiment LWC-WO LWC-RG MWC-WO SC TMP-N0.01 TMP-No.02 Newsprint

$\begin{array}{cccccccc}1 & 1.28 & 0.83 & 0.67 & 1.31 & 3.9 & 3.2 & 3.76 \\ 2 & 1.38 & 0.93 & 0.67 & 1.04 & 4.3 & 3.28 & 3.46 \\ 3 & 1.05 & 0.84 & 0.63 & 1.27 & 3.66 & 3.02 & 3.8 \\ 4 & 1.08 & 0.68 & 0.72 & 1.06 & 4.54 & 2.94 & 3.63 \\ 5 & 0.96 & 0.82 & 0.61 & 1.19 & 4.44 & 3.85 & 3.3 \\ 6 & 1.06 & 0.61 & 0.75 & 1.14 & 3.86 & 2.7 & 3.35 \\ & & & & & & & \\ \text { Averape } & 1.135 & 0.785 & 0.675 & 1.168333 & 4.116667 & 3.165 & 3.55 \\ \text { Stco. Dev. } & 0.159719 & 0.117431 & 0.05282 & 0.109438 & 0.357417 & 0.392823 & 0.211471\end{array}$

Note: Measured area: $603 \times 459 \mu \mathrm{m}$; scanning depth: $100 \mu \mathrm{m}$. 
Appendix 2. Roughness ( $R a, u m)$ measured using stylus scanning profilometry

\begin{tabular}{|c|c|c|c|c|c|c|c|c|c|c|c|c|}
\hline Expt. & TMP No.1 & TMP NO.2 & 2 TMP No.3 & TMP No.4 & New: & $\mathbf{s s}$ & 18 & sc & LWC-RO & LWC-Wo & MWC-Wo & Remark \\
\hline 1 & 2.8 & 2.89 & 2.82 & 3.03 & 3.1 & 3.07 & 2.66 & 1.42 & 1 & 1.15 & 0.88 & MD \\
\hline 2 & 3.17 & 3.11 & 2,36 & 3,12 & 2.8 & 2.71 & 2.51 & 1.24 & 0.93 & 1.02 & 0.7 & MD \\
\hline 3 & 3.24 & 2.69 & 2.37 & 2.69 & 2.56 & 3.02 & 2.54 & 1.28 & 0.89 & 1.04 & 0.71 & MD \\
\hline 4 & 3.58 & 2.7 & 2.85 & 2.95 & 2.82 & 3.16 & 2.57 & 1.32 & 1 & 1.17 & 0.71 & MD \\
\hline 5 & 3.17 & 2.81 & 2.63 & 3.02 & 3.11 & 2.83 & 2.53 & 1.28 & 0.85 & 1.01 & 0.68 & MD \\
\hline 6 & 2.8 & 2.42 & 2,18 & 2.93 & 2.71 & 2.75 & 2.25 & 1.17 & 1.17 & 0.86 & 0.71 & $M D$ \\
\hline 7 & 3,8 & 2.74 & 2.3 & 2.86 & 2.67 & 2.47 & 2.49 & 1.5 & 0.98 & 1.13 & 0.67 & $M D$ \\
\hline 8 & 2.89 & 2.88 & 2.69 & 3.41 & 3.37 & 2.75 & 2.45 & 1.23 & 0.98 & 1.16 & 0.75 & $M D$ \\
\hline 8 & 3.72 & 3.22 & 2.51 & 2.91 & 3 & 2.54 & 2.82 & 1.24 & 1.03 & 0.93 & 0.63 & MD \\
\hline 10 & 3.03 & 2.28 & 2.48 & 3.24 & 2,94 & 2.82 & 2.38 & 1.18 & 0.73 & 1.1 & 0.81 & $M D$ \\
\hline 11 & 3.29 & 2.93 & 2.87 & 3.36 & 3.31 & 3.16 & 2.43 & 1.41 & 1.07 & 1.06 & 0.67 & CD \\
\hline 12 & 3.82 & 3.24 & 2.98 & 3.07 & 3 & 2.93 & 2.66 & 1.1 & 1.07 & 0.88 & 0.67 & CD \\
\hline 13 & 3.85 & 2.8 & 3.28 & 3,34 & 3.04 & 3.28 & 2.62 & 1.25 & 1.13 & 1.03 & 0.68 & $C D$ \\
\hline 14 & 3.52 & 2.77 & 2.57 & 2.78 & 2.96 & 3.61 & 2.78 & 1.3 & 1.09 & 1.18 & 0,65 & CD \\
\hline 15 & 3.26 & 2.92 & 2.74 & 2.98 & 3.36 & 3.45 & 2.83 & 1.26 & 1.16 & 1.1 & 0.66 & $C D$ \\
\hline 16 & 3.82 & 2.7 & 2.34 & 3.57 & 3.13 & 3.11 & 3.06 & 1.37 & 1.23 & 1.14 & 0.69 & $C D$ \\
\hline 17 & 3.26 & 2.92 & 2.68 & 2.89 & 3.44 & 3.1 & 2.93 & 1.19 & 1.04 & 1.24 & 0.75 & $C D$ \\
\hline 18 & 3.58 & 2.71 & 2.43 & 3.09 & 3.04 & 3.25 & 2.86 & 1.14 & 1.01 & 1.13 & 0.87 & CD \\
\hline 18 & 3.71 & 2.71 & 2,88 & 3.09 & 2.97 & 3.04 & 2.71 & 1.36 & 0.96 & 1.08 & 0.69 & CD \\
\hline 20 & 3.46 & 2,69 & 2.78 & 3.43 & 3.16 & 3.06 & 2.63 & 1.23 & 1.1 & 1.02 & 0.83 & CD \\
\hline Average & 3.3935 & 2.8065 & 2.642 & 3.103 & 3.0245 & 3.0055 & 2.6355 & 1.2735 & 1.021 & 1.077 & 0.7205 & \\
\hline Sid. Dev. & 0.33915 & 0.23002 & 0.27739 & 0.21781 & 0.23809 & 0.28714 & 0.20119 & 0.10059 & 0.11715 & 0.09171 & 0.07251 & \\
\hline
\end{tabular}


Appendix 3. Experimental data of WYKO repeatability test

$\begin{array}{cccccc}\text { Experiment } & \mathbf{R}_{\mathbf{a}} & \mathbf{R}_{\mathbf{q}} & \mathbf{R} \text { ku } & \mathbf{R}_{\mathbf{s}} & \text { Ave. Step Ht } \\ 1 & 3.23 & 4.63 & 9.64 & 0.78 & -62.79 \\ 2 & 3.19 & 4.57 & 9.21 & 0.77 & -62.95 \\ 3 & 3.21 & 4.51 & 9.47 & 0.8 & -62.83 \\ 4 & 3.2 & 4.53 & 9.35 & 0.77 & -62.75 \\ 5 & 3.25 & 4.69 & 9.69 & 0.79 & -62.76 \\ 5 & 3.22 & 4.55 & 9.61 & 0.78 & -62.91 \\ 7 & 3.22 & 4.56 & 9.53 & 0.76 & -62.92 \\ 8 & 3.23 & 4.58 & 9.57 & 0.77 & -62.85 \\ 9 & 3.22 & 4.57 & 9.66 & 0.77 & -62.37 \\ 10 & 3.22 & 4.56 & 9.38 & 0.77 & -62.88 \\ \text { Average } & 3.219 & 4.575 & 9.511 & 0.776 & -62.801 \\ \text { Std. Dev. } & 0.0166333 & 0.05126185 & 0.1563081 & 0.01173788 & 0.16596184\end{array}$

Note: Sample: TMP No.1; scanning area: $603 \times 459$ um; captured area: $320 \times 320$ um.

Appendix 4. Fiber-to-fiber bond strength (J/M2) of uncoated papers

$\begin{array}{llllll}\text { Experiment } & \text { SS } & \text { IS } & \text { SC } & \text { TMP No.1 TMP N0.2 News }\end{array}$

$\begin{array}{ccccccc}1 & 72.72 & 56.35 & 47.24 & 48.41 & 45.96 & 49.88 \\ 2 & 72.72 & 56.25 & 44.1 & 49 & 49.88 & 49.88 \\ 3 & 75.66 & 56.15 & 44.49 & 49.29 & 49.1 & 49.69 \\ 4 & 69.68 & 51.74 & 44.2 & 50.67 & 48.41 & 47.33 \\ 5 & 71.93 & 54.88 & 45.18 & 49.39 & 49.2 & 49 \\ 6 & 68.4 & 53.02 & 44.69 & 46.94 & 49.29 & 49.69\end{array}$

$\begin{array}{lllllll}\text { Average } & 71.8516667 & 54.7316667 & 44.9833333 & 48.95 & 48.64 & 49.245\end{array}$

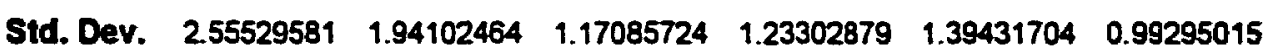


Appendix 5. Changes in $\mathrm{Ra}, \mathrm{Rq}$, and average step height due to pure water rewertting (sample: MWC-WO; 2-point line rewetting)

\begin{tabular}{|c|ccc|cccc|ccc|}
\hline Experiment & \multicolumn{3}{|c|}{ R.um } & \multicolumn{3}{c|}{ Rg.um } & \multicolumn{3}{c|}{ Ave. step HL, um } \\
\hline & Before & Anter & Change & Before & After & Change & Before & Arter & Change \\
\hline 1 & 0.49 & 0.64 & 0.15 & 0.63 & 0.81 & 0.18 & -57.77 & 56.1 & 1.67 \\
2 & 0.41 & 0.91 & 0.5 & 0.53 & 1.13 & 0.6 & -59.17 & 57.23 & 1.94 \\
3 & 0.57 & 1.26 & 0.69 & 0.74 & 1.59 & 0.85 & .59 .65 & 58.52 & 1.13 \\
4 & 0.43 & 0.77 & 0.34 & 0.58 & 0.98 & 0.4 & -58.34 & 57.16 & 1.18 \\
5 & 0.51 & 0.98 & 0.47 & 0.68 & 0.2 & -0.48 & -56.45 & 55.49 & 0.96 \\
6 & 0.45 & 0.72 & 0.27 & 0.61 & 0.96 & 0.35 & 56.72 & 55.46 & 1.26 \\
& & & & & & & & & \\
Average & & & 0.403333 & & & 0.316667 & & & 1.356657 \\
Std. Dev. & & & 0.190753 & & & 0.453019 & & & 0.370873 \\
\hline
\end{tabular}

Note: Scanning field: $603 \times 459$ um; scarning depth: 100 um; captured paper area: $320 \times 320$ um.

Appendix 6. Changes in roughness and average step height due to rewetting of 2-time printig

Appendix 6.1. Changes in Ra, Rq, and average step height due to rewetting of 2-time printig (sample: MWC-WO: 2-point line rewetting)

\begin{tabular}{|c|ccc|ccc|ccc|}
\hline \multirow{2}{*}{ Experiment } & \multicolumn{3}{|c|}{ Ra, um } & \multicolumn{3}{c|}{ Rq, um } & \multicolumn{3}{|c|}{ Ave. step Ht. um } \\
& Before & After & Change & Before & After & Change & Before & After & Change \\
1 & 0.6 & 1.01 & 0.41 & 0.79 & 1.33 & 0.54 & -58.07 & 56.46 & 1.61 \\
2 & 0.72 & 0.91 & 0.19 & 0.79 & 1.03 & 0.24 & -59.24 & 57.29 & 1.95 \\
3 & 0.51 & 1.08 & 0.57 & 0.64 & 1.43 & 0.79 & -60.63 & -58.62 & 2.01 \\
4 & 0.59 & 0.82 & 0.23 & 0.73 & 1.44 & 0.71 & -61.48 & -50.63 & 0.85 \\
5 & 0.63 & 1.16 & 0.53 & 0.77 & 1.33 & 0.56 & -6292 & -61.13 & 1.79 \\
6 & 0.54 & 1.12 & 0.58 & 0.65 & 1.03 & 0.38 & 59.66 & -57.94 & 1.72 \\
& & & & & & & & & \\
Average & & & 0.418333 & & & 0.536657 & & & 1.655 \\
Std. Dev. & & & 0.172791 & & & 0.203634 & & & 0.420844 \\
\hline
\end{tabular}

Note: Scanning fiekd: $603 \times 459$ um; scanning depth: 100 um; captured paper area: $320 \times 320$ um; wetting liquid: ink-dyed water (20\% ink). 
Appendix 6.2. Changes in Ra, Rq, and average step height due to rewetting of 2-time printig (sample: SC; 2-point line rewetting: printing 2 times)

\begin{tabular}{|c|c|c|c|c|c|c|c|c|c|}
\hline \multirow[t]{2}{*}{ Experiment } & \multicolumn{3}{|c|}{ Ra, um } & \multicolumn{3}{|c|}{ Ra. um } & \multicolumn{3}{|c|}{ Ave stop Ht, um } \\
\hline & Eefore & Anter & Change & Before & After & Change & Before & Aftor & Change \\
\hline 1 & 0.96 & 221 & 1.25 & 0.29 & 2.75 & 2.46 & 61.54 & -57.45 & 4.00 \\
\hline 2 & 0.93 & 2.05 & 1.12 & 1.33 & 2.51 & 1.18 & 61.02 & +57.22 & 3.8 \\
\hline 3 & 1.02 & 2.36 & 1.34 & 1.37 & 2.57 & 1.2 & 62.82 & $\mathbf{5 0 . 3}$ & 9.52 \\
\hline 4 & 1.05 & 2.43 & 1.38 & T.16 & 3.12 & 1.96 & -6.00 & $.00,35$ & 5.74 \\
\hline 5 & 0.91 & 2.57 & 1.66 & 1.23 & 2.98 & 1.75 & $\$ 2.76$ & -55.34 & 7.42 \\
\hline 6 & 0.93 & 276 & 1.83 & 1.25 & 2.82 & 1.57 & 64.21 & $\$ 7.59$ & 6.62 \\
\hline Average & & & 1.43 & & & 1.686657 & & & 6.198333 \\
\hline Sid Der. & & & 0.26533 & & & 0.486607 & & & 2149925 \\
\hline
\end{tabular}

Note: Scanning field: $603 \times 459$ um; scanning depth: 100 um; captured paper area: $320 \times 320$ um.

Appendix 6.3. Changes in $\mathrm{Ra}, \mathrm{Rq}$, and average step height due to rewetting of 2-time printig (sample: Newsprint; 2-point line rewetting; printing 2 times)

\begin{tabular}{|c|c|c|c|c|c|c|c|c|c|}
\hline \multirow[t]{2}{*}{ Experiment } & \multicolumn{3}{|c|}{ Raum } & \multicolumn{3}{|c|}{ Ra, um } & \multicolumn{3}{|c|}{ Ave. step Ht. um } \\
\hline & Before & After & Change & Before & After & Change & Before & Anter & Change \\
\hline 1 & 3.17 & 4.99 & 1.82 & 4.11 & 6.64 & 2.53 & -65.63 & -61.13 & 4.7 \\
\hline 2 & 3.08 & 3.79 & 0.73 & 3.98 & 5.02 & 1.04 & $-\infty .93$ & 65.45 & 4.40 \\
\hline 3 & 3.05 & 3.99 & 0.94 & 3.97 & 5.24 & 1.27 & -68.03 & 62.38 & 5.65 \\
\hline 4 & 4.02 & 5.48 & 1.46 & 5.39 & 6.77 & 1.38 & -57.79 & -52.89 & 49 \\
\hline $\mathbf{5}$ & 3.25 & 4.60 & 1.38 & 4.66 & 6.34 & 1.68 & -62.97 & -58.46 & 4.51 \\
\hline 6 & 2.95 & 436 & 0.41 & 3.55 & 4.97 & 1.42 & -62.01 & 50.32 & 3.69 \\
\hline Average & & & 1.29 & & & 1.553333 & & & 4.655 \\
\hline Sid. Der. & & & 0.39202 & & & 0.52103 & & & 0.637958 \\
\hline
\end{tabular}

Note: Scannirg field: $603 \times 459$ um; scanning depth: 100 un; captured paper area; $320 \times 320$ um; metting liquid: ink-dyed water (20\% ink). 
Appendix 7. Changes in roughness, skewness, kurtosis, and average step height due to rewetting

Appendlx 7.1. Changes in roughness, skewness, kurtosis, and average step height due to rewetting (sample: MWC-WO; 2-point line rewelting; wetting liquid: $20 \%$ ink-dyed water)

\begin{tabular}{|c|c|c|c|c|c|c|c|c|c|c|c|c|c|c|c|}
\hline \multirow[b]{2}{*}{ Expt } & \multicolumn{3}{|c|}{ Ra, um } & \multicolumn{3}{|c|}{$R q, u m$} & \multicolumn{3}{|c|}{ Rku } & \multicolumn{3}{|c|}{ Rsk } & \multicolumn{3}{|c|}{ Ave step Ht, um } \\
\hline & Before & After & Dit. & Before & After & Dif. & Before & After & Dit. & Before & After & Dif. & Before & After & Dit. \\
\hline 1 & 0.43 & 0.64 & 0.21 & 0.57 & 0.83 & 0.26 & 4.74 & 3.46 & -1.3 & -0.07 & 0.14 & 0.21 & -59.06 & -58 & 0.57 \\
\hline 2 & 0.53 & 0.84 & 0.31 & 0.72 & 1.08 & 0.36 & 9.92 & 6.18 & -3.7 & -1.27 & 0.21 & 1.48 & -60.13 & -58 & 225 \\
\hline 3 & 0.44 & 1.09 & 0.65 & 0.62 & 1.43 & 0.81 & 6.77 & 10.9 & 4.1 & .0 .56 & 1.21 & 1.77 & -59.98 & -59 & 1.27 \\
\hline 4 & 0.4 & 0.69 & 0.29 & 0.53 & 0.91 & 0.38 & 5.64 & 3.87 & -1.8 & -0.82 & 0.06 & 0.88 & | -59.92 & -58 & 1.88 \\
\hline 5 & 0.6 & 1.04 & 0.44 & 0.74 & 1.29 & 0.55 & 3.33 & 3.01 & -0.3 & .0 .42 & 0.08 & 0.5 & -60.23 & -59 & 1.19 \\
\hline 5 & 0.57 & 0.73 & 0.16 & 0.81 & 1 & 0.19 & 6.65 & 5 & -1.7 & -0.78 & 0.03 & 0.81 & 61.62 & -61 & 0.6 \\
\hline Average & 0.495 & 0.84 & 0.34 & 0.665 & 1.09 & 0.43 & 6.175 & 5.4 & -0.8 & -0.65 & 0.29 & 0.94 & -60.16 & .59 & 1.29 \\
\hline Sid. Dev & 0.083 & 0.19 & 0.18 & 0.109 & 0.23 & 0.22 & 2.238 & 2.92 & 2.64 & 0.407 & 0.46 & 0.59 & 0.829 & 1.14 & 0.67 \\
\hline
\end{tabular}

Note: Scanning field $603 \times 459$ um; scanning depth: 100 um; captured paper area: $320 \times 320$ um.

Appendix 7.2. Changes in roughness, skewness, kurtosis, and average step height due to rewetting (sample: LWC-WO; 2-point line rewetting; wetting liquid: $20 \%$ ink-dyed water)

\begin{tabular}{|c|c|c|c|c|c|c|c|c|c|c|c|c|c|c|c|}
\hline \multirow[b]{2}{*}{ Expt } & \multicolumn{3}{|c|}{ Ra, um } & \multicolumn{3}{|c|}{$\mathrm{Rq}, \mathrm{um}$} & \multicolumn{3}{|c|}{ Rku } & \multicolumn{3}{|c|}{ Rsk } & \multicolumn{3}{|c|}{ Ave. step Ht, um } \\
\hline & Before & Atter & Dif. & Before & After & Dif. & Before & After & Dif. & Before & After & & Before & After & Dif. \\
\hline 1 & 0.9 & 1.39 & 0.49 & 1.13 & 1.81 & 0.68 & 3.28 & 4.77 & 1.49 & -0.42 & 0.46 & 0.88 & -57.95 & -54 & 3.65 \\
\hline 2 & 0.62 & 0.89 & 0.27 & 0.79 & 1.18 & 0.39 & 3.55 & 4.77 & 1.21 & -0.09 & 0.46 & 0.55 & .62 .3 & -61 & 1.53 \\
\hline 3 & 0.85 & 1.26 & 0.41 & 1.19 & 1.68 & 0.49 & 9.49 & 4.6 & -4.9 & -1.47 & -0 & 1.44 & .62 .55 & -60 & 2.56 \\
\hline 4 & 1.04 & 1.89 & 0.85 & 1.31 & 2.34 & 1.03 & 3.36 & 3.63 & 0.27 & -0.08 & 0.1 & 0.18 & -6272 & -60 & 2.25 \\
\hline 5 & 0.73 & 1.37 & 0.64 & 0.95 & 1.75 & 0.8 & 4.65 & 4.22 & -0.4 & -0.57 & 0.29 & 0.86 & -59.47 & -57 & 2.89 \\
\hline 6 & 0.72 & 1.09 & 0.29 & 0.98 & 1.34 & 0.36 & 5.63 & 4.98 & -0.5 & -0.96 & -0.3 & 0.7 & -60.92 & -58 & 2.62 \\
\hline Average & 0.81 & 1.3 & 0.49 & 1.058 & 1.68 & 0.63 & 4.995 & 4.5 & -0.5 & -0.6 & 0.17 & 0.77 & -60.99 & -58 & 258 \\
\hline tid. Dev & 0.15 & 0.35 & 0.22 & 0.187 & 0.41 & 0.26 & 2.393 & 0.49 & 2.31 & 0.539 & 0.29 & 0.42 & 1.934 & 255 & 0.7 \\
\hline
\end{tabular}

Note: Scanning field: $603 \times 459$ um; scanning depth: 100 um; captured paper area: $320 \times 320$ um. 
Appendix 7.3. Changes in roughness, skewness, kurtosis, and average step height due to rewetting (sample: LWC-RG; 2-point line rewelting; wetting liquid: $20 \%$ ink-dyed water)

\begin{tabular}{|c|c|c|c|c|c|c|c|c|c|c|c|c|c|c|c|}
\hline \multirow[b]{2}{*}{ Expt } & \multicolumn{3}{|c|}{ Ra, um } & \multicolumn{3}{|c|}{$\mathrm{Rq}$, um } & \multicolumn{3}{|c|}{ Rku } & \multicolumn{3}{|c|}{ Rsk } & \multicolumn{3}{|c|}{ Ave. step Ht, um } \\
\hline & Before & After & Dif. & Before & After & Dif. & Before & After & Dif. & Before & After & Dif. & Before & After & Dif. \\
\hline 1 & 0.9 & 1.66 & 0.76 & 1.1 & 2.07 & 0.97 & 2.85 & 3.25 & 0.4 & -0.01 & 0.39 & 0.4 & $|-56.8|$ & -55 & 2.17 \\
\hline 2 & 0.61 & 1.03 & 0.42 & 0.77 & 1.36 & 0.59 & 3.28 & 5.14 & 1.86 & -0.32 & 0.94 & 1.26 & -57.9 & -57 & 1.36 \\
\hline 3 & 0.61 & 1.17 & 0.56 & 0.76 & 1.45 & 0.69 & 3 & 2.85 & -0.2 & 0.22 & 0.42 & 0.2 & -57.8 & -56 & 1.62 \\
\hline 4 & 0.77 & 1.39 & 0.62 & 0.95 & 1.72 & 0.77 & 2.76 & 2.76 & 0 & -0.09 & 0.19 & 0.28 & -57.1 & -55 & 1.83 \\
\hline 5 & 0.52 & 1.14 & 0.62 & 0.67 & 1.45 & 0.78 & 3.35 & 3.21 & -0.1 & -0.08 & 0.3 & 0.38 & -57.9 & -56 & 2.33 \\
\hline 6 & 0.53 & 1.03 & 0.5 & 0.68 & 1.36 & 0.68 & 4.08 & 4.88 & 0.8 & -0.27 & 0.44 & 0.71 & -60.2 & -58 & 1.92 \\
\hline Average & 0.657 & 1.24 & 0.58 & 0.822 & 1.57 & 0.75 & 3.22 & 3.68 & 0.46 & -0.09 & 0.45 & 0.54 & -58 & .56 & 1.87 \\
\hline d. Dev. & 0.149 & 0.25 & 0.12 & 0.169 & 0.28 & 0.13 & 0.481 & 1.05 & 0.78 & 0.19 & 0.26 & 0.39 & 1.189 & 1.26 & 0.35 \\
\hline
\end{tabular}

Note: Scanning field: $603 \times 459$ um; scanning depth: 100 um; captured paper area: $320 \times 320$ um.

Appendix 7.4. Changes in roughness, skewness, kurtosis, and average step height due to rewetting (sample: SC; 2-point line rewetting; wetting liquid: $20 \%$ ink-dyed water)

\begin{tabular}{|c|c|c|c|c|c|c|c|c|c|c|c|c|c|c|c|}
\hline \multirow[b]{2}{*}{ Expt } & \multicolumn{3}{|c|}{ Ra, um } & \multicolumn{3}{|c|}{ Rq, um } & \multicolumn{3}{|c|}{ Rku } & \multicolumn{3}{|c|}{ Rsk } & \multicolumn{3}{|c|}{ Ave. step Ht, um } \\
\hline & Before & After & Dif. & Before & After & Dif. & Before & After & Dif. & Before & After & Dif. & Before & Atter & Dif. \\
\hline 1 & 1.07 & 2.19 & 1.12 & 1.38 & 2.76 & 1.38 & 7.89 & 5.39 & -2.5 & $\mid-0.62$ & 0.13 & 0.75 & \begin{tabular}{|l|l|} 
& -58.2
\end{tabular} & -52 & 6.3 \\
\hline 2 & 0.78 & 1.61 & 0.83 & 1 & 2 & 1 & 5.76 & 3.53 & -2.2 & -0.76 & -0 & 0.73 & -58 & -53 & 4,62 \\
\hline 3 & 1.03 & 2.39 & 1.36 & 1.55 & 3.13 & 1.58 & 5.65 & 10.3 & 4.69 & $\mid-1.59$ & -0.3 & 1.28 & -58.1 & .52 & 6.2 \\
\hline 4 & 0.93 & 1.78 & 0.85 & 1.25 & 2.26 & 1.01 & 5.63 & 3.61 & -2 & -0.43 & -0.2 & 0.22 & $\mid-57.2$ & .54 & 3.53 \\
\hline 5 & 0.98 & 2.27 & 1.29 & 1.41 & 2.86 & 1.45 & 6.53 & 5.97 & -0.6 & $|-0.31|$ & $0.2 t$ & 0.58 & \begin{tabular}{|l|}
-57.8 \\
\end{tabular} & -51 & 6.42 \\
\hline 6 & 0.98 & 1.7 & 0.72 & 1.31 & 2.35 & 1.04 & 5.66 & 4.45 & -1.2 & -0.73 & 0.25 & 0.98 & -58 & -53 & 4.96 \\
\hline rerage & 0.962 & 1.99 & 1.03 & 1.317 & 2.56 & 1.24 & 6.187 & 5.55 & -0.6 & -0.74 & 0.02 & 0.76 & -57.9 & -53 & 5.34 \\
\hline d $\cap 0$ & 0.101 & 0.33 & 0.27 & 0.185 & 0.42 & 0.26 & 0.903 & 2.54 & 271 & 0.45 & 0.24 & 0.36 & 0.352 & 0.95 & 1.16 \\
\hline
\end{tabular}

Note: Scanning field: $603 \times 459$ um; scanning depth: 100 um; captured paper area: $320 \times 320$ um. 
Appendix 7.5. Changes in roughness, skewness, kurtosis, and average step height due to rewetting (sample: Surface sized copy paper; 2-point line rewetting; wetting liquid: $20 \%$ ink-dyed water)

\begin{tabular}{|c|c|c|c|c|c|c|c|c|c|c|c|c|c|c|c|}
\hline \multirow[b]{2}{*}{ Expt } & \multicolumn{3}{|c|}{ Ra, um } & \multicolumn{3}{|c|}{ Rq, um } & \multicolumn{3}{|c|}{ Rku } & \multicolumn{3}{|c|}{ Rsk } & \multicolumn{3}{|c|}{ Ave. step Ht. um } \\
\hline & Before & After & Dif. & Before & After & Dif. & Before & After & Dif. & Before & After & Dif. & Before & Anter & Dif. \\
\hline 1 & 4.29 & 4.56 & 0.27 & 4.27 & 5.06 & 0.79 & 5.09 & 8.91 & 3.82 & 0.19 & 1.25 & 1.06 & -56.9 & -51 & 5.81 \\
\hline 2 & 3.61 & 4.94 & 1.33 & 5.17 & 6.41 & 1.24 & 9.95 & 4.11 & -5.8 & 1.72 & 0.93 & -0.8 & -60.2 & -56 & 3.73 \\
\hline 3 & 3.46 & 5.99 & 2.53 & 4.67 & 7.87 & 3.2 & 8.22 & 4.64 & -3.6 & 1.18 & 1.06 & -0.1 & -61.9 & -57 & 4.77 \\
\hline 4 & 3.74 & 4.28 & 0.54 & 5.05 & 5.61 & 0.56 & 6.95 & 4.35 & -2.6 & 0.89 & 0.63 & -0.3 & -62.6 & -60 & 2.87 \\
\hline 5 & 4.56 & 5.75 & 1.19 & 5.02 & 5.95 & 0.93 & 9.23 & 3.01 & -6.2 & 0.07 & 0.99 & 0.92 & -59.3 & -56 & 3.49 \\
\hline 6 & 4.13 & 4.58 & 0.45 & 5.62 & 5.73 & 0.11 & 6.48 & 3.25 & -3.2 & 1.1 & 1.38 & 0.28 & -55.9 & -54 & 1.89 \\
\hline Average & 3.965 & 5.02 & 1.05 & 4.967 & 6.11 & 1.14 & 7.653 & 4.71 & -29 & 0.86 & 1.04 & 0.18 & $\mid-59.4$ & -56 & 3.76 \\
\hline id. Dev. & 0.429 & 0.7 & 0.84 & 0.459 & 0.97 & 1.08 & 1.818 & 2.15 & 3.62 & 0.63 & 0.26 & 0.72 & 2.65 & 291 & 1.38 \\
\hline
\end{tabular}

Note: Scanning field: $603 \times 459$ um; scanning depth: 100 um; captured paper area: $320 \times 320$ um.

Appendix 7.6. Changes in roughness, skewness, kurtosis, and average step height due to rewetting (sample: Internally sized copy paper, 2-point line rewetting; wetting liquid: $20 \%$ ink-dyed water)

\begin{tabular}{|c|c|c|c|c|c|c|c|c|c|c|c|c|c|c|c|}
\hline \multirow[b]{2}{*}{ Expt. } & \multicolumn{3}{|c|}{$\mathrm{Ra}_{\mathrm{a}}$ um } & \multicolumn{3}{|c|}{ Rq, um } & \multicolumn{3}{|c|}{ Rku } & \multicolumn{3}{|c|}{ Rsk } & \multicolumn{3}{|c|}{ Ave. step Ht, um } \\
\hline & Before & Anter & Dif. & Before & Anter & Dif. & Before & After & Dif. & Before & After & Dif. & Before & After & Dit. \\
\hline 1 & 4.03 & 5.18 & 1.15 & 3.99 & 5.14 & 1.15 & 6.87 & 9.14 & 2.27 & 0.15 & 0.56 & 0.41 & -61.4 & -57 & 4.12 \\
\hline 2 & 3.81 & 4.45 & 0.64 & 3.39 & 4.29 & 0.9 & 4.75 & 6.1 & 1.35 & 0.21 & 0.39 & 0.18 & -58.4 & -54 & 4.48 \\
\hline 3 & 3.72 & 4.54 & 0.82 & 3.31 & 4.28 & 0.97 & 5.67 & 4.31 & -1.4 & 0.37 & 0.94 & 0.57 & -65.1 & -60 & 5.02 \\
\hline 4 & 2.96 & 4.47 & 1.51 & 3.25 & 4.66 & 1.41 & 7.62 & 5.31 & -2.3 & 0.62 & 0.41 & -0.2 & -62.7 & -59 & 3.87 \\
\hline 5 & 2.83 & 4.58 & 1.75 & 3.55 & 5.31 & 1.76 & 8.24 & 5.66 & -2.6 & 0.55 & 1.22 & 0.67 & -59.5 & -57 & 2.27 \\
\hline 6 & 4.15 & 5.67 & 1.52 & 3.57 & 5.65 & 2.08 & 5.77 & 4.23 & -1.5 & 0.73 & 0.51 & -0.2 & -60.8 & -54 & 6.61 \\
\hline Average & 3.583 & 4.82 & 1.23 & 3.51 & 4.89 & 1.38 & 6.487 & 5.79 & -0.7 & 0.44 & 0.67 & 0.23 & -61.3 & -57 & 4.4 \\
\hline itd. Dev. & 0.556 & 0.5 & 0.44 & 0.267 & 0.57 & 0.47 & 1.319 & 1.8 & 2.01 & 0.23 & 0.33 & 0.38 & 2.381 & 2.47 & 1.43 \\
\hline
\end{tabular}

Note: Scanning field: $603 \times 459$ um; scanning depth: 100 um; captured paper area: $320 \times 320$ um. 
Appendix 7.7. Changes in roughness, skewness, kurtosis, and average step height due to rewetting (sample: TMP No.01;2-point line rewetting; wetting liquid: 20\% ink-dyed water)

\begin{tabular}{|c|c|c|c|c|c|c|c|c|c|c|c|c|c|c|c|}
\hline \multirow[b]{2}{*}{ Expt. } & \multicolumn{3}{|c|}{ Ra, um } & \multicolumn{3}{|c|}{ Rq, um } & \multicolumn{3}{|c|}{ Rku } & \multicolumn{3}{|c|}{ Rsk } & \multicolumn{3}{|c|}{ Avo. step $\mathrm{Ht}$, um } \\
\hline & Before & After & Dif. & Before & After & Dif. & Before & After & Dif. & Before & Ater & Dif. & Before & Aner & Dif. \\
\hline 4 & 2.59 & 4.4 & 1.81 & 3.54 & 5.72 & 2.18 & 8.98 & 4.96 & -4 & 0.75 & 0.81 & 0.06 & -76.5 & -75 & 1.89 \\
\hline 2 & 3.22 & 4.57 & 1.35 & 4.52 & 6.08 & 1.56 & 10.09 & 4.64 & -5.5 & 0.48 & 0.69 & 0.21 & -60.8 & .55 & 5.8 \\
\hline 3 & 3.56 & 4.57 & 1.01 & 4.62 & 5.84 & 1.22 & 5.74 & 4.15 & -1.6 & 0.31 & 0.32 & 0.01 & -64 & -59 & 4.86 \\
\hline 4 & 3.96 & 5.33 & 1.37 & 7.35 & 8.88 & 1.53 & 3.98 & 2.73 & -1.3 & 0.44 & 0.38 & -0.1 & .63 .5 & .61 & 2.41 \\
\hline 5 & 3.87 & 5.79 & 1.92 & 5.01 & 7.47 & 2.46 & 5.34 & 3.88 & -1.5 & 0.62 & 0.79 & 0.17 & -63.3 & -57 & 6.34 \\
\hline 6 & 4.33 & 5.11 & 0.78 & 5.61 & 6.5 & 0.89 & 5.79 & 4.32 & -1.5 & 0.64 & 0.54 & -0.1 & -64.2 & -58 & 6.18 \\
\hline Average & 3.588 & 4.96 & 1.373 & 5.108 & 6.75 & 1.64 & 6.653 & 4.11 & .2 .5 & 0.54 & 0.59 & 0.05 & -65.4 & 81 & 4.58 \\
\hline dd, Dev. & 0.617 & 0.54 & 0.441 & 1.291 & 1.22 & 0.59 & 2.353 & 0.78 & 1.76 & 0.16 & 0.21 & 0.12 & 5.595 & 7.08 & 1.96 \\
\hline
\end{tabular}

Note: Scanning field: $603 \times 459$ um; scanning depth: 100 um; captured paper area: $320 \times 320$ um.

Appendix 7.8. Changes in roughness, skewness, kurtosis, and average step height due to rewetting (sample: TMP No.02; 2-point line rewetting; wetting liquid: $20 \%$ ink-dyed water)

\begin{tabular}{|c|c|c|c|c|c|c|c|c|c|c|c|c|c|c|c|}
\hline \multirow[b]{2}{*}{ Expt } & \multicolumn{3}{|c|}{$\mathrm{Ra}, \mathrm{um}$} & \multicolumn{3}{|c|}{ Rq, um } & \multicolumn{3}{|c|}{ Rkut } & \multicolumn{3}{|c|}{ Rsk } & \multicolumn{3}{|c|}{ Ave. step Ht, um } \\
\hline & Before & Atter & Dif. & Before & After & Dif. & Before & After & Dif. & Before & After & Dif. & Before & After & Dif. \\
\hline 1 & 262 & 4.2 & 1.58 & 3.46 & 5.41 & 1.95 & 3.85 & 7.14 & 3.29 & 0.44 & 0.57 & 0.13 & -66.4 & -61 & 4.93 \\
\hline 2 & 3.23 & 4.38 & 1.15 & 4.35 & 5.78 & 1.43 & 6.43 & 4.75 & -1.7 & -0.16 & -0 & 0.13 & -59.9 & -57 & 2.49 \\
\hline 3 & & 4.78 & 1.25 & 4.57 & 6.11 & 1.54 & $4.6 !$ & 3.43 & -1.2 & 0.15 & 0.17 & 0.02 & -66.1 & -62 & 4.54 \\
\hline 4 & 2.74 & 3.63 & 1.09 & 3.73 & 5.07 & 1.34 & 6.88 & 4.49 & -2.4 & -0.02 & 0.38 & 0.4 & .59 .8 & .55 & 4.39 \\
\hline 5 & 3.36 & 5.85 & 249 & 4.61 & 7.54 & 2.93 & 8.42 & 4.25 & -4.2 & 0.59 & 0.47 & -0.1 & -68.4 & 62 & 3.89 \\
\hline 6 & 1.8 & 3.97 & 2.17 & 2.42 & 5.49 & 3.07 & 9.02 & 6.04 & -3 & 0.27 & 0.99 & 0.72 & -63.7 & -56 & 8.11 \\
\hline Average & 2.88 & 4.5 & 1.522 & 3.857 & 5.9 & 2.04 & 6.535 & 5.02 & -1.5 & 0.21 & 0.43 & 0.21 & -63.7 & -59 & 4.73 \\
\hline td. Dev. & 0.637 & 0.74 & 0.583 & 0.843 & 0.88 & 0.77 & 2.038 & 1.34 & 2.58 & 0.28 & 0.35 & 0.3 & 3.148 & 3.21 & 1.86 \\
\hline
\end{tabular}

Note: Scanning field. $603 \times 459$ um; scanning depth: 100 um; captured paper area: $320 \times 320$ um. 
Appendix 7.9. Changes in roughness, skewness, kurtosis, and average step height due to rewetting (sample: Newsprint; 2-point line rewetting; wetting liquid: $20 \%$ ink-dyed water)

\begin{tabular}{|c|c|c|c|c|c|c|c|c|c|c|c|c|c|c|c|}
\hline \multirow[b]{2}{*}{ Expt. } & \multicolumn{3}{|c|}{ Ra, um } & \multicolumn{3}{|c|}{ Rq. um } & \multicolumn{3}{|c|}{ Rku } & \multicolumn{3}{|c|}{ Rsk } & \multicolumn{3}{|c|}{ Ave. step Ht, um } \\
\hline & Before & After & Dif. & Before & After & Dif. & Before & After & Dif. & Before & After & Dit. & Before & Ater & Dif. \\
\hline 1 & 2.78 & 3.73 & 0.95 & 3.85 & 5.07 & 1.22 & 9.11 & 8.47 & .0 .6 & -0.05 & 0.51 & 0.56 & -66 & -62 & 4.12 \\
\hline 2 & 3.4 & 4.97 & 1.57 & 5.01 & 7.14 & 2.13 & 9.36 & 6.38 & -3 & 1.08 & 9.12 & 0.04 & -68.3 & -62 & 6.57 \\
\hline 3 & 2.94 & 3.46 & 0.52 & 4.21 & 4.77 & 0.56 & 12.25 & 9.79 & -2.5 & 0.93 & 0.94 & 0.01 & -65.4 & -62 & 2.95 \\
\hline 4 & 2.95 & 3.94 & 0.99 & 3.71 & 5.01 & 1.3 & 4.77 & 4.92 & 0.15 & 0.34 & 0.6 & 0.26 & -55 & -52 & 2.7 \\
\hline 5 & 2.73 & 3.6 & 0.87 & 3.47 & 4.55 & 1.08 & 5.43 & 4.45 & -1 & 0.44 & 0.57 & 0.13 & $\mid-55.1$ & -52 & 2.95 \\
\hline 6 & 3.93 & 4.85 & 0.92 & 5.16 & 6.4 & 1.24 & 6.02 & 5.64 & -0.4 & 0.65 & 0.87 & 0.22 & -61.7 & -60 & 1.96 \\
\hline Average & 3.122 & 4.09 & 0.97 & 4.235 & 5.49 & 1.26 & 7.823 & 6.61 & -1.2 & 0.57 & 0.77 & 0.2 & -61.9 & -58 & 3.54 \\
\hline Std. Dev. & 0.461 & 0.65 & 0.339 & 0.702 & 1.04 & 0.51 & 2.895 & 2.1 & 1.23 & 0.41 & 0.24 & 0.2 & 5.736 & 4.87 & 1.64 \\
\hline
\end{tabular}

Note: Scanning field: $603 \times 459$ um; scanning depth: 100 um; captured paper area: $320 \times 320$ um.

Appendix 8. Changes in roughness, skewness, kurtosis, and average step height due to 50 um droplet rewetting

Appendix 8.1. Changes in roughness, skewness, kurtosis, and average step height due to rewetting (sample: MWC-WO; 50 um droplet rewetting; wetting liquid: $20 \%$ ink-dyed water)

\begin{tabular}{|c|c|c|c|c|c|c|c|c|c|c|c|c|c|c|c|}
\hline \multirow[b]{2}{*}{ Expt. } & \multicolumn{3}{|c|}{ Ra, um } & \multicolumn{3}{|c|}{$\mathrm{Rq}, \mathrm{um}$} & \multicolumn{3}{|c|}{ Rku } & \multicolumn{3}{|c|}{ Rsk } & \multicolumn{3}{|c|}{ Ave. step Ht, um } \\
\hline & Before & After & Dif. & Eefore & After & Dif. & Before & After & Dif. & Before & Anter & Dif. & Before & Anter & Dif. \\
\hline 1 & 0.23 & 0.26 & 0.03 & 0.31 & 0.37 & 0.06 & 7.09 & 6.06 & -1 & -0.8 & -1 & -0.2 & -58.2 & -58 & 0.69 \\
\hline 2 & 0.49 & 0.53 & 0.04 & 0.69 & 0.76 & 0.07 & 9.21 & 8.16 & -1.1 & 0.15 & 0.71 & 0.56 & -60.3 & -59 & 0.87 \\
\hline 3 & 0.32 & 0.38 & 0.06 & 0.47 & 0.56 & 0.09 & 6.5 & 5.17 & -1.3 & 0.28 & 0.74 & 0.46 & -57.7 & .57 & 0.56 \\
\hline 4 & 0.35 & 0.32 & -0.03 & 0.44 & 0.4 & -0 & 7.24 & 4.58 & -2.7 & -0.34 & 0.26 & 0.6 & -59.4 & -59 & 0.55 \\
\hline 5 & 0.41 & 0.49 & 0.08 & 0.53 & 0.58 & 0.05 & 4.07 & 3.26 & -0.8 & 0.25 & 0.36 & 0.11 & -60.5 & -60 & 0.59 \\
\hline 6 & 0.28 & 0.36 & 0.08 & 0.37 & 0.45 & 0.08 & 3.58 & 4.51 & 0.93 & -0.11 & 0.09 & 0.2 & -61.8 & -61 & 0.95 \\
\hline Average & 0.347 & 0.39 & 0.043 & 0.468 & 0.52 & 0.05 & 6.282 & 5.29 & -1 & -0.1 & 0.2 & 0.29 & $\mid-59.6$ & -59 & 0.7 \\
\hline Std. Dev. & 0.093 & 0.1 & 0.041 & 0.133 & 0.14 & 0.05 & 2116 & 1.68 & 1.15 & 0.42 & 0.63 & 0.3 & 1.526 & 1.42 & 0.17 \\
\hline
\end{tabular}


Appendix 8.2. Changes in roughness, skewness, kurtosis, and average step height due to rewelting (sample: LWC-WO; 2-point line rewetting; wetting liquid: $20 \%$ ink-dyed water)

\begin{tabular}{|c|c|c|c|c|c|c|c|c|c|c|c|c|c|c|c|}
\hline \multirow[b]{2}{*}{ Expt } & \multicolumn{3}{|c|}{ Ra, um } & \multicolumn{3}{|c|}{ Rq, um } & \multicolumn{3}{|c|}{ Rku } & \multicolumn{3}{|c|}{ Rsk } & \multicolumn{3}{|c|}{ Ave. step Ht, um } \\
\hline & Before & Ater & Dif. & Before & After & Dif. & Eefore & Anter & Dif. & Before & Anter & Dif. & Before & Anter & Dif. \\
\hline 1 & 0.63 & 0.74 & 0.11 & 0.78 & 0.91 & 0.13 & 4.71 & 4.32 & -0.4 & .0 .3 & -0.2 & 0.09 & -55.2 & .54 & 1.2 \\
\hline 2 & 0.54 & 0.6 & 0.06 & 0.72 & 0.8 & 0.08 & 2.79 & 2.67 & -0.1 & -0.21 & 0.45 & 0.66 & -60 & .59 & 1.13 \\
\hline 3 & 0.49 & 0.57 & 0.08 & 0.62 & 0.7 & 0.08 & 3.52 & 266 & -0.9 & -0.77 & -0.4 & 0.33 & -50.5 & -49 & 1.83 \\
\hline 4 & 0.6 & 0.75 & 0.15 & 0.73 & 0.89 & 0.16 & 3.23 & 2.52 & -0.7 & 0.29 & 1.25 & 1.04 & -65.8 & -64 & 1.88 \\
\hline 5 & 0.45 & 0.53 & 0.08 & 0.64 & 0.85 & 0.21 & 3.36 & 2.65 & -0.7 & -0.8 & -0.3 & 0.51 & -58.8 & -57 & 1.66 \\
\hline 6 & 0.83 & 1.02 & 0.19 & 1.25 & 1.37 & 0.12 & 4.36 & 3.13 & -1.2 & 0.35 & 0.35 & 0 & 61.7 & -60 & 1.87 \\
\hline Average & 0.59 & 0.7 & 0.11 & 0.79 & 0.92 & 0.13 & 3.662 & 2.99 & -0.7 & -0.25 & 0.19 & 0.44 & -58.7 & -57 & 1.6 \\
\hline Std. Dev. & 0.135 & 0.18 & 0.05 & 0.233 & 0.23 & 0.05 & 0.727 & 0.68 & 0.38 & 0.48 & 0.63 & 0.39 & 5.306 & 5.25 & 0.34 \\
\hline
\end{tabular}

Note: Scanning field: $603 \times 459$ um; scanning depth: $100 \mathrm{um}$; captured paper area: $80 \times 80 \mathrm{um}$.

Appendix 8.3. Changes in roughness, skewness, kurtosis, and average step height due to rewetting (sample: LWC-RG; 50 um droplet rewetting; wetting liquid: 20\% ink-dyed water)

\begin{tabular}{|c|c|c|c|c|c|c|c|c|c|c|c|c|c|c|c|}
\hline \multirow[b]{2}{*}{ Expt } & \multicolumn{3}{|c|}{ Ra, um } & \multicolumn{3}{|c|}{ Rq, um } & \multicolumn{3}{|c|}{ Rku } & \multicolumn{3}{|c|}{ Rsk } & \multicolumn{3}{|c|}{ Ave. step Ht. um } \\
\hline & Before & After & Dif. & Before & After & Dif. & Before & After & Dif. & Before & After & Dif. & Before & Atter & Dif. \\
\hline 1 & 0.48 & 0.55 & 0.07 & 0.78 & 0.91 & 0.13 & 5.29 & 5.61 & 0.32 & -0.93 & -0.8 & 0.14 & -58.9 & -57 & 1.39 \\
\hline 2 & 0.28 & 0.62 & 0.34 & 0.37 & 1.12 & 0.75 & 4.11 & 3.53 & -0.6 & -0.66 & 0.33 & 0.99 & 62.7 & -62 & 0.98 \\
\hline 3 & 0.39 & 0.43 & 0.04 & 0.45 & 0.58 & 0.13 & 7.58 & 6.16 & -1.4 & 0.39 & 0.8 & 0.41 & -55.3 & .54 & 1.18 \\
\hline 4 & 0.4 & 0.51 & 0.11 & 0.53 & 0.67 & 0.14 & 7.44 & 3.54 & -3.9 & -0.3 & 0.16 & 0.46 & -63.9 & -63 & 0.69 \\
\hline 5 & 0.42 & 0.5 & 0.08 & 0.49 & 0.58 & 0.09 & 4.14 & 3.86 & -0.3 & -0.35 & -0.1 & 0.28 & -64.8 & -64 & 0.9 \\
\hline 6 & 0.4 & 0.37 & -0.03 & 0.52 & 0.46 & -0.1 & 3.94 & 3.5 & -0.4 & -0.3 & -0 & 0.27 & -65.6 & -65 & 0.96 \\
\hline Average & 0.395 & 0.5 & 0.1 & 0.523 & 0.72 & 0.2 & 5.417 & 4.37 & -1.1 & -0.36 & 0.07 & 0.43 & -61.9 & -61 & 1.02 \\
\hline Std. Dev. & 0.065 & 0.09 & 0.13 & 0.139 & 0.25 & 0.28 & 1.692 & 1.2 & 1.5 & 0.44 & 0.52 & 0.3 & 3.982 & 4.16 & 0.24 \\
\hline
\end{tabular}

Note: Scanning field $603 \times 459$ um; scanning depth: 100 um; captured paper area: $80 \times 80$ um. 
Appendix 8.4. Changes in roughness, skewness, kurtosis, and average step height due to rewetting (sample: SC; 50 um droplet rewetting; wetting liquid: 20\% ink-dyed water)

\begin{tabular}{|c|c|c|c|c|c|c|c|c|c|c|c|c|c|c|c|}
\hline \multirow[b]{2}{*}{ Expt. } & \multicolumn{3}{|c|}{ Ra, um } & \multicolumn{3}{|c|}{$\mathrm{Rq}, \mathrm{um}$} & \multicolumn{3}{|c|}{ Rku } & \multicolumn{3}{|c|}{ Rsk } & \multicolumn{3}{|c|}{ Ave. step Ht. um } \\
\hline & Before & After & Dif. & Before & After & Dif. & Before & After & Dif. & Before & After & Dif. & Before & Anter & Dif. \\
\hline 1 & 0.92 & 1.19 & 0.27 & 1.32 & 1.63 & 0.31 & 5.95 & 10.2 & 4.21 & 1.8 & 2.1 & 0.3 & -63.9 & 62 & 2.31 \\
\hline 2 & 0.63 & 0.65 & 0.02 & 0.87 & 0.92 & 0.05 & 9.04 & 6.42 & -2.6 & -1.4 & -1.7 & -0.3 & -66.4 & 64 & 2.07 \\
\hline 3 & 1.05 & 1.57 & 0.52 & 1.42 & 1.97 & 0.55 & 13.66 & 15.4 & 1.75 & 1.28 & 0.03 & -1.3 & 61.9 & .58 & 3.47 \\
\hline 4 & 0.9 & 1 & 0.1 & 1.19 & 1.53 & 0.34 & 21.12 & 10.8 & -10 & 0.04 & 0.95 & 0.91 & -67.4 & -65 & 2.52 \\
\hline 5 & 0.75 & 0.86 & 0.11 & 0.99 & 1.18 & 0.19 & 6.08 & 6.02 & -0.1 & -1.13 & -0.7 & 0.46 & -67.1 & -64 & 2.71 \\
\hline 6 & 0.54 & 0.71 & 0.17 & 0.89 & 1.12 & 0.23 & 20.38 & 8.94 & -11 & -2.72 & 0.77 & 3.49 & -59.4 & -57 & 2.1 \\
\hline erage & 0.798 & 1 & 0.198 & 1.113 & 1.39 & 0.28 & 12.71 & 9.63 & -3.1 & -0.36 & 0.24 & 0.6 & -64.3 & -62 & 2.53 \\
\hline d. Dev. & 0.193 & 0.34 & 0.178 & 0.231 & 0.39 & 0.17 & 6.835 & 3.43 & 6.45 & 1.72 & 1.34 & 1.6 & 3.315 & 3.38 & 0.52 \\
\hline
\end{tabular}

Note: Scanning field: $603 \times 459$ um; scanning depth: 100 um; captured paper area: $80 \times 80$ um.

Appendix 8.5. Changes in roughness, skewness, kurtosis, and average step height due to rewelting (sample: Surface sized copy paper, 50 um droplet rewetting; wetting liquid: $20 \%$ ink-dyed water)

\begin{tabular}{|c|c|c|c|c|c|c|c|c|c|c|c|c|c|c|c|}
\hline \multirow[b]{2}{*}{ Expt. } & \multicolumn{3}{|c|}{ Ra, um } & \multicolumn{3}{|c|}{ Rq, um } & \multicolumn{3}{|c|}{ Rku } & \multicolumn{3}{|c|}{ Rsk } & \multicolumn{3}{|c|}{ Ave. step $\mathrm{HL}, \mathrm{um}$} \\
\hline & Before & After & Dif. & Before & After & Dif. & Before & After & Dif. & Before & Anter & Dif. & Before & Anter & Dif. \\
\hline 1 & 25 & 2.58 & 0.08 & 3.25 & 3.44 & 0.19 & 10.26 & 8.61 & -1.7 & 1.39 & 1.56 & 0.17 & $\mid-58.8$ & -58 & 1 \\
\hline 2 & 2.95 & 3 & 0.05 & .45 & 4.55 & 0.1 & & 11.4 & -1 & 1.98 & 2.16 & 0.18 & -56.9 & -56 & 1.35 \\
\hline 3 & 2.17 & 2.31 & 0.14 & 2.92 & 3.19 & 0.27 & 14.55 & 12.7 & -1.9 & 1.07 & 1.62 & 0.55 & -77.3 & .76 & 1.1 \\
\hline 4 & 3.27 & 3.52 & 0.25 & 4.36 & 4.8 & 0.44 & 6.94 & 5.65 & -1.3 & 0 & 0.35 & 0.35 & -63.8 & -62 & 1.37 \\
\hline 5 & 3.06 & 3.25 & 0.19 & 4.35 & 4.53 & 0.18 & 9.14 & 7.54 & -1.6 & 0.29 & 0.42 & 0.13 & -58.4 & -57 & 1.51 \\
\hline 6 & 2.45 & 2.87 & 0.42 & 3.36 & 3.58 & 0.22 & 11.91 & 9.78 & -2.1 & 3.77 & 4.83 & 1.06 & -61.2 & -59 & 2.27 \\
\hline Average & 2733 & 292 & 0.188 & 3.782 & 4.02 & 0.23 & 10.87 & 9.28 & -1.6 & 1.42 & 1.82 & 0.41 & -627 & -61 & 1.43 \\
\hline id. Dev. & 0.423 & 0.44 & 0.135 & 0.679 & 0.69 & 0.12 & 2.675 & 2.57 & 0.41 & 1.36 & 1.64 & 0.36 & 7.526 & 7.65 & 0.45 \\
\hline
\end{tabular}

Note: Scanning field: $603 \times 459$ um; scanning depth: 100 um; captured paper area: $80 \times 80$ um. 
Appendix 8.6. Changes in roughness, skewness, kurtosis, and average step height due to rewetting (sample: Internally sized copy paper, 50 um droplet rewetting; wetting liquid: $20 \%$ ink-dyed water)

\begin{tabular}{|c|c|c|c|c|c|c|c|c|c|c|c|c|c|c|c|}
\hline \multirow[b]{2}{*}{ Expt } & \multicolumn{3}{|c|}{$\mathbf{R a}$, um } & \multicolumn{3}{|c|}{ Rq. um } & \multicolumn{3}{|c|}{ Rku } & \multicolumn{3}{|c|}{ Rsk } & \multicolumn{3}{|c|}{ Ave. step Ht., um } \\
\hline & efore & After & Dif. & Before & After & Dif. & Before & After & Dif. & Before & Aftar & Dif. & Before & Atter & Dif. \\
\hline$t$ & 2.94 & 3.05 & 0.11 & 3.73 & 4 & 0.27 & 6.31 & 4.06 & -2.3 & 0.34 & 0.79 & 0.45 & -62.4 & .58 & 4.0 \\
\hline 2 & 2.32 & 3.11 & 0.79 & 3.18 & 4.12 & 0.94 & 9.12 & 7.67 & -1.5 & 0.62 & 1.07 & 0.45 & -58.4 & -55 & 3.1 \\
\hline 3 & 3.17 & 3.28 & 0.11 & 4.08 & 4.25 & 0.17 & 5.46 & 5.39 & -0.1 & 0.85 & 1.74 & 0.89 & -67.6 & -65 & 2.2 \\
\hline 4 & 1.72 & 1.85 & 0.13 & 2.37 & 2.61 & 0.24 & 9.38 & 6.01 & -3.4 & 1 & 1.78 & 0.78 & -63.4 & -61 & 21 \\
\hline 5 & 298 & 3.24 & 0.26 & & 3.82 & 0.28 & & 5.66 & -1.4 & & 0.96 & 0.41 & -54.6 & .53 & 20 \\
\hline 6 & 3.08 & 3.33 & 0.25 & 3.45 & 3.61 & 0.16 & 8.95 & 7.04 & -1.9 & 0.77 & 1.46 & 0.69 & -65.3 & -63 & 23 \\
\hline Average & 2.702 & 2.98 & 0.28 & 3.392 & 3.74 & 0.34 & 7.712 & 5.97 & -1.7 & 0.69 & 1.3 & 0.61 & -61.9 & -59 & 2.66 \\
\hline d. Dev. & 0.567 & 0.56 & 0.26 & 0.583 & 0.6 & 0.3 & 1.66 & 1.27 & 1.09 & 0.23 & 0.42 & 0.2 & 4.728 & 4.83 & 0.8 \\
\hline
\end{tabular}

Note: Scanning field: $603 \times 459$ um; scanning depth: $100 \mathrm{um}$; captured paper area: $80 \times 80$ um.

Appendix 8.7. Changes in roughness, skewness, kurtosis, and average step height due to rewelting (sample: TMP NO.01; 50 um droplet rewetting; wetting lquid: $20 \%$ ink-dyed water)

\begin{tabular}{|c|c|c|c|c|c|c|c|c|c|c|c|c|c|c|c|}
\hline \multirow[b]{2}{*}{ Expt } & \multicolumn{3}{|c|}{$\mathbf{R a}$, um } & \multicolumn{3}{|c|}{ Rq, um } & \multicolumn{3}{|c|}{ Rku } & \multicolumn{3}{|c|}{ Rsk } & \multicolumn{3}{|c|}{ Ave step Hth, um } \\
\hline & Before & After & Dif. & Before & Anter & Dif. & Before & After & Dif. & Before & After & Dif. & Before & Anter & Dif. \\
\hline 1 & 1.71 & 2.06 & 0.35 & 252 & 3.2 & 0.68 & 21.31 & 20.4 & -0.9 & 1.25 & 2.07 & 0.82 & -69.6 & -68 & 2.03 \\
\hline 2 & $\$ .72$ & 1.89 & 0.17 & 251 & 26 & 0.09 & 12.96 & 13.5 & 0.53 & 269 & $\$ .12$ & -1.6 & -77.7 & .76 & 1.44 \\
\hline 3 & 1.97 & 2.31 & 0.34 & 2.58 & 3.03 & 0.45 & 7.53 & 5.05 & -2.5 & -0.03 & 0.38 & 0.41 & .73 .5 & -70 & 3.06 \\
\hline 4 & 2.77 & 3.08 & 0.31 & 3.77 & 4.12 & 0.35 & 11.1 & 9.62 & -1.5 & 1.42 & 2.47 & 1.05 & -62.5 & -61 & 1.76 \\
\hline 5 & 1.72 & 2.35 & 0.63 & 245 & 3.32 & 0.87 & 21.59 & 14.8 & -6.8 & 21 & 2.23 & 0.13 & -64.4 & .63 & 1.23 \\
\hline 6 & 2.53 & 294 & 0.41 & 1.97 & 2.5 & 0.53 & 14.06 & 11.5 & -2.5 & 0.73 & 1.19 & 0.46 & -60.9 & -59 & 1.89 \\
\hline Average & 2.07 & 244 & 0.37 & 2633 & 3.13 & 0.5 & 14.76 & 125 & -23 & 1.36 & 1.58 & 0.22 & -68.1 & -66 & 1.9 \\
\hline Std. Dev. & 0.466 & 0.48 & 0.15 & 0.599 & 0.58 & 0.27 & 5.638 & 5.16 & 2.47 & 0.97 & 0.81 & 0.93 & 6.677 & 6.54 & 0.64 \\
\hline
\end{tabular}

Note: Scanning feld: $603 \times 459 \mathrm{um}$; scanning depth: $100 \mathrm{um}$; captured paper area: $80 \times 80 \mathrm{um}$. 
Appendix 8.8. Changes in roughness, skewness, kurtosis, and average step height due to rewetting (sample: TMP NO.02; 50 um droplet rewetting; wetting liquid: 20\% ink-dyed water)

\begin{tabular}{|c|c|c|c|c|c|c|c|c|c|c|c|c|c|c|c|}
\hline \multirow[b]{2}{*}{ Expt. } & \multicolumn{3}{|c|}{ Ra, um } & \multicolumn{3}{|c|}{ Rq, um } & \multicolumn{3}{|c|}{ Rku } & \multicolumn{3}{|c|}{ Rsk } & \multicolumn{3}{|c|}{ Ave step Ht., um } \\
\hline & Before & Anter & Dif. & Before & After & Dif. & Before & After & Dif. & Before & Anter & Dif. & Before & After & Dif. \\
\hline 1 & 2.03 & 2.41 & 0.38 & 284 & 3.21 & 0.37 & $\begin{array}{l}7.25 \\
\end{array}$ & 13.1 & 5.8 & 0.02 & 0.2 & 0.18 & \begin{tabular}{|l|} 
\\
\end{tabular} & -57 & 1.52 \\
\hline 2 & 1.97 & 2.16 & 0.19 & 2.76 & 3.12 & 0.36 & 9.8 & 8.74 & -1.1 & -0.88 & -0.3 & 0.58 & -67.4 & -66 & 1.04 \\
\hline 3 & 3.08 & 3.56 & 0.48 & 4.23 & 4.87 & 0.64 & 8.67 & 9.03 & 0.36 & -1.59 & -1.3 & 0.3 & -55.4 & -53 & 2.63 \\
\hline 4 & 2.54 & 2.76 & 0.22 & 3.38 & 3.67 & 0.29 & 26.63 & 8.23 & -18 & 0.61 & 0.75 & 0.14 & -57.3 & -54 & 3.23 \\
\hline 5 & 2.21 & 2.48 & 0.27 & 2.88 & 3.31 & 0.43 & 7.71 & 5.73 & -2 & 0.27 & 0.55 & 0.28 & -61.7 & .58 & 3.27 \\
\hline 6 & 2.5 & 2.97 & 0.47 & 3.42 & 3.81 & 0.39 & 7.58 & 6.33 & -1.3 & 0.87 & 1.28 & 0.41 & -59.5 & -57 & 2.12 \\
\hline Average & 2.388 & 2.72 & 0.335 & 3.252 & 3.67 & 0.41 & 11.27 & 8.52 & -2.8 & -0.12 & 0.2 & 0.32 & -59.9 & -58 & 2.3 \\
\hline Std. Dev. & 0.412 & 0.5 & 0.126 & 0.557 & 0.65 & 0.12 & 7.58 & 2.59 & 8.17 & 0.94 & 0.9 & 0.16 & 4.223 & 4.77 & 0.91 \\
\hline
\end{tabular}

Note: Scanning field: $603 \times 459$ um; scanning depth: 100 um; captured paper area: $80 \times 80$ um.

Appendix 8.9. Changes in roughness, skewness, kurtosis, and average step height due to rewetting (sample: Newsprint; 50 um droplet rewetting; wetting liquid: $20 \%$ ink-dyed water)

\begin{tabular}{|c|c|c|c|c|c|c|c|c|c|c|c|c|c|c|c|}
\hline \multirow[b]{2}{*}{ Expt } & \multicolumn{3}{|c|}{ Ra, um } & \multicolumn{3}{|c|}{$R q$, um } & \multicolumn{3}{|c|}{ Rku } & \multicolumn{3}{|c|}{ Rsk } & \multicolumn{3}{|c|}{ Ave step Ht, um } \\
\hline & Before & After & Dif. & Before & After & Dif. & Before & After & Dif. & Before & After & Dif. & Before & Ater & Dif. \\
\hline 1 & 2.3 & 2.51 & 0.21 & 3.52 & 3.87 & 0.35 & 20.74 & 16.4 & .4 .4 & 2.33 & 2.43 & 0.1 & -65.8 & -63 & 2.91 \\
\hline 2 & 2.77 & 3.1 & 0.33 & 4.33 & 4.72 & 0.39 & 13.02 & 15 & 2 & 1.11 & 1.68 & 0.57 & -60.2 & .57 & 3.09 \\
\hline 3 & 3.66 & 3.81 & 0.15 & 4.4 & 4.61 & 0.21 & 2.59 & 8.94 & 6.35 & 0.23 & 1.38 & 1.15 & -57.1 & -56 & 1.48 \\
\hline 4 & 1.94 & 2.34 & 0.4 & 2.63 & 3.71 & 1.08 & 12.13 & 3.56 & .8 .6 & 0.23 & -0.2 & -0.4 & -50.5 & -48 & 1.99 \\
\hline 5 & 1.68 & 1.77 & 0.09 & 2.2 & 2.38 & 0.18 & 5.78 & 6.98 & 1.2 & 2.91 & 3.65 & 0.74 & -55.1 & -53 & 2.25 \\
\hline 6 & 1.5 & 1.58 & 0.08 & 1.88 & 2.12 & 0.24 & 12.13 & 3.56 & -8.6 & 1.4 & 1.13 & -0.3 & -61.7 & -60 & 1.44 \\
\hline Average & 2308 & 252 & 0.21 & 3.16 & 3.57 & 0.41 & 11.07 & 9.07 & -2 & 1.37 & 1.69 & 0.32 & $\mid-58.4$ & -56 & 2.19 \\
\hline Std. Dev. & 0.803 & 0.83 & 0.131 & 1.084 & 1.1 & 0.34 & 6.316 & 5.55 & 6.13 & 1.09 & 1.28 & 0.6 & 5.369 & 5.16 & 0.7 \\
\hline
\end{tabular}

Note: Scanning field: $603 \times 459$ um; scanning depth: $100 \mathrm{um}$; captured paper area: $80 \times 80 \mathrm{um}$. 